\title{
Navier-Stokes Equations for Stochastic Particle Systems on the Lattice
}

\author{
R. Esposito ${ }^{1, \star}$, R. Marra ${ }^{2, \star \star}$, H.T. Yau ${ }^{3, \star \star \star}$ \\ ${ }^{1}$ Dipartimento di Matematica Pura ed Applicata, Università di L'Aquila, Coppito, AQ, Italy \\ ${ }^{2}$ Dipartimento di Fisica, Università di Roma Tor Vergata, Via della Ricerca Scientifica, 00133 \\ Roma, Italy \\ ${ }^{3}$ Courant Institute, 251 Mercer Street, New York NY 10012, USA
}

Received: 19 June 1995/Accepted: 8 May 1996

\begin{abstract}
We introduce a class of stochastic models of particles on the cubic lattice $\mathbb{Z}^{d}$ with velocities and study the hydrodynamical limit on the diffusive spacetime scale. Assuming special initial conditions corresponding to the incompressible regime, we prove that in dimension $d \geqq 3$ there is a law of large numbers for the empirical density and the rescaled empirical velocity field. Moreover the limit fields satisfy the corresponding incompressible Navier-Stokes equations, with viscosity matrices characterized by a variational formula, formally equivalent to the Green-Kubo formula.
\end{abstract}

\section{Introduction}

One of the main open problems in nonequilibrium statistical physics is the derivation of the hydrodynamical equations of fluids from the microscopic Hamiltonian dynamics. The main fluid equations are the Navier-Stokes equations and the Euler equations. The Euler equations represent the conservation of macroscopic mass, energy and momentum and have an obvious hyperbolic scaling invariance

$$
x \rightarrow \alpha x, \quad t \rightarrow \alpha t .
$$

The Navier-Stokes equations are more complicated and have no obvious scaling. They are given by correcting the Euler equations with viscous terms described by second order derivatives of conserved quantities such as energy, momentum and mass. In the incompressible regime, the Navier-Stokes equations become

$$
\begin{gathered}
\operatorname{div} u=0, \\
\partial_{t} u+u \cdot \nabla u+\nabla p=v \Delta u
\end{gathered}
$$

with $u$ the velocity field, $p$ the pressure and $v>0$ the kinematic viscosity.

\footnotetext{
$\star$ Partially supported by GNFM-CNR and MURST.

$\star \star$ Partially supported by GNFM-CNR, INFN and MURST.

$\star \star \star$ Partially supported by U.S. National Science Foundation grant 9403462 and David and Lucile Packard Foundation Fellowship.
} 
Since Euler and Navier-Stokes equations involve macroscopic quantities, derivations of these equations from microscopic Hamiltonian dynamics are understood in the sense of law of large numbers, as the number of particles tends to infinity. We fix the space and time scales by choosing the typical interparticle distance as the unit length scale and the mean free time of particles as unit time scale. Suppose the range of molecular interactions are of the order of the typical interparticle distance. Then each particle typically interacts with an at most finite number of nearby particles. Let $\varepsilon$ denote the ratio between the microscopic and macroscopic length units. In order that particles travel a unit macroscopic length in a unit macroscopic time, the macroscopic time scale is chosen to be $\varepsilon^{-1} t$. The derivation of Euler equations can be stated as proving that the Euler equations are exact in the scaling limit $x \rightarrow \varepsilon^{-1} x, t \rightarrow \varepsilon^{-1} t$. This scaling is usually called the Euler scale or hyperbolic scaling.

We now sketch Morrey's idea [1] for the derivation of the Euler equations. Since the Hamiltonian dynamics is conservative, the time derivatives of conserved quantities are given by microscopic conservation laws characterized by the corresponding microscopic currents. The currents involve microscopic interactions of particles and are not functions of local conserved quantities. To obtain closed equations, one has to represent these currents as functions of the local conserved quantities through some law of large numbers. If we assume that the system is locally in equilibrium and described by Gibbs states characterized by local conserved quantities, from the law of large numbers for Gibbs states these currents can be replaced by their expectations with respect to Gibbs states depending on local conserved quantities. Hence we obtain closed equations.

The basic ingredient in the previous heuristic derivation is a strong ergodic theorem for Hamiltonian systems. Though this ergodic theorem is a basic problem in the theory of Hamiltonian systems and is very easy to state, without further assumption, it is nevertheless still an open question. If certain random perturbations are added to the Hamiltonian dynamics, or if certain assumptions are made, it can be rigorously proved [2]. Alternatively, if instead of Hamiltonian dynamics one starts from the Boltzmann equation, very detailed derivations of Euler equations are available $([3,4,5])$. Of course the Boltzmann equation is not a microscopic equation and, because the Boltzmann equation describes the low density regime, the state equation obtained in this case is just the one of the perfect gases. It should also be noted that all these derivations are valid only up to the times such that the Euler equations have smooth solutions.

The similar program for the Navier-Stokes equations is much harder to carry out. First of all, the Navier-Stokes equations have no obvious scaling and thus they cannot be a scaling limit. Furthermore, although the basic equations in the classical physics are hyperbolic and completely reversible, the Navier-Stokes equations are irreversible due to the viscosity.

The first difficulty is more of a technical nature and there are several possible solutions. One of them is to consider the following incompressible limit. The diffusive effects, like the viscosity and the heat conduction are hard to detect, because they are small corrections to the Euler equations. They become relevant on the diffusive scale, i.e.

$$
x \rightarrow \varepsilon^{-1} x, \quad t \rightarrow \varepsilon^{-2} t .
$$

The Navier-Stokes equations are not invariant under such scaling, so the prescription of the diffusive scaling (1.3) cannot be used in full generality. In order to restore 
the scaling invariance one needs to scale also the velocity field $u$ as

$$
u \rightarrow \varepsilon u,
$$

and, consequently, the pressure as $p \rightarrow \bar{p}+\varepsilon^{2} p$ with $\bar{p}$ an arbitrary constant. In other words, we consider a regime where macroscopic velocities are very small compared with the sound speed. To summarize, the incompressible Navier-Stokes equations (1.2) can be understood as the scaling limit under the the rules given by (1.3) and (1.4). This limit is called the incompressible limit. It is used in $[6,7]$ to derive the incompressible Navier-Stokes equations (1.2) from the Boltzmann equation and in [8] to derive the viscous Burgers equation as a diffusive limit of the simple exclusion process.

The second difficulty is a deep conceptual question and relates to the origin of diffusivity in classical physics. Up to now, there is no good mathematical understanding for a derivation of diffusive behavior from reversible systems. A heuristic explanation, however, can be given. We follow [9] where a formal derivation of the equations (1.2) from the Hamiltonian systems are given. From Morrey's heuristic argument or from the recent work of [2], the many body Liouville equation can be approximately solved using an ansatz of local Gibbs states, i.e., Gibbs states with slowly varying chemical potentials. Let us strengthen the local equilibrium assumption by demanding that the Liouville equation can be solved asymptotically in orders of $\varepsilon$. Then locally the solution of the Liouville equation is given by a Gibbs state with an $\varepsilon$ order correction. Therefore, the currents should be replaced by their expectations with respect to this state. The leading order contribution to the currents will be the same as in the Euler equation case; the $\varepsilon$ order correction will give rise to the viscosity. The difficulties in carrying out this approach rigorously are obvious. First of all, there is no rigorous proof that local Gibbs states give even the leading order solution of the Liouville equation unless some noise are added to the Liouville equation. Furthermore, it is in general hard to quantify the meaning of the asymptotic expansion involving an infinite number of variables. One way to make sense of this asymptotic expansion is through the use of relative entropy $[2,10]$. But lack of analytic tools for general Hamiltonian systems force us to consider lattice gases models.

The models we consider are defined as follows. Let $\mathscr{V}$ be a finite set of vectors in $\mathbb{R}^{d}$ of fixed length $\kappa>0$, invariant under reflections and exchanges of the coordinate axes. It will represent the set of the possible velocities of the particles. For each $v \in \mathscr{V}$ we consider a species of particles moving on the lattice $\mathbb{Z}^{d}$ according to a simple exclusion process (SEP), with jump intensities such that the resulting drift is $v$. This means that a particle of the species $v$ in a site $x \in \mathbb{Z}^{d}$ jumps with exponential law to one of the neighboring sites $x+e \in \mathbb{Z}^{d},|e|=1$, with intensity $p_{e}(v)$, if $x+e$ is not already occupied by a particle with velocity $v$. We denote by $\eta(x, v)=0,1$ the occupation number of particles of the species $v$ in $x$. The intensities $p_{e}(v)$ are chosen so that the drift of the process for the $v$-particles is $v$. The jumps of each particle are independent of the particles with different velocities. They are also independent of the particles with the same velocity, as far as the exclusion condition is satisfied. The changes of velocity are due to collisions. Particles collide when they are in the same site and have suitable velocities. The essential condition on the collisions is that they conserve the number of particles involved in the collision and their total momentum, and nothing else. In the next section we define two models, called Model I and Model II, by giving the set $\mathscr{V}$ and the collision rules. 
Our method can be extended to a wider class of models and also to more general lattices.

We take the set $\mathscr{V}$ for Model I to be the smallest possible by including only the $2 d$ unit vectors parallel to the coordinate directions; for the Model II the set $\mathscr{V}$ includes several vectors with non-coordinate directions to make the model as isotropic as possible. In both cases the jump intensity is chosen of the form

$$
p_{e}(v)=\chi+\frac{1}{2} v \cdot e,
$$

with $\chi$ big enough to make positive all the jump intensities. Note that we allow a particle with velocity $v$ to jump also in "wrong" directions, as the one orthogonal to $v$. This is convenient to prove ergodicity, but the assumption that such a jump happens with intensity bigger than $\kappa / 2$ is unnecessary: any positive intensity would work.

As for the collisions, we consider binary collisions, which exchange the velocities of any pair of particles at the same site into another pair of velocities having the same total momentum chosen at random among all the possible ones (if any), provided that the exclusion condition is not violated. The evolution of our model will be described in terms of a generator $\mathscr{L}$ which will be the sum of the exclusion part $\mathscr{L}^{e x}$ and the collision part $\mathscr{L}^{c}$.

Lattice gas models for the hydrodynamics have been widely considered before. We refer to [11] and references quoted therein for a comprehensive review. The aim of these works was to construct simple models for hydrodynamical equations which could be carefully and quickly simulated on the computer. Hence the effort is to make them as deterministic as possible. The numerical results provided a remarkably good agreement with hydrodynamical equations. The macroscopic behaviors were also analyzed theoretically, under the assumption of propagation of chaos. This produced a type of Boltzmann equation for the models, although no explicit low density condition was introduced and no proofs were given. After adding a small amount of stochasticity, the Euler regime was studied in [12] using the approach of [2]. In $[13,14]$ the Euler and Navier-Stokes regimes were considered for models with discrete space, time and velocities, and with stochastic free motion and deterministic or stochastic collisions. However the kinetic limit was used as an intermediate step. In the present paper we deal directly with the hydrodynamic limit and no intermediate step or low density assumption is needed.

As discussed before, our analysis is confined to the incompressible regime. The incompressibility assumption corresponds to choose the chemical potentials of the local equilibrium suitably small, i.e. of order $\varepsilon$. Following the heuristic derivation of viscosity in the previous paragraph, we have to compute the next order correction term of this state, which is of order $\varepsilon^{2}$. This is the key element of this paper. The viscosity will be determined through this correction term.

This analysis allows us to prove our main result, the validity of the law of large numbers for the empirical density and the rescaled empirical velocity field as the scaling parameter $\varepsilon$ goes to 0 . In fact, Theorem 2.1 states their weak convergence in probability to deterministic fields solving the incompressible Navier-Stokes equation with the viscosity determined according to the previous argument. A very short sketch of the idea of the proof is the following.

Recall the dynamics has two parts: the exclusion part $\mathscr{L}^{e x}$ and the collision part $\mathscr{L}^{c}$. Without the collisions our process is just the asymmetric simple exclusion process. The parallel problem for the asymmetric simple exclusion processes has 
been studied in [8] using the spectral gap and the logarithmic Sobolev inequality. Unfortunately, these estimates are hard to prove for the present models. We use an alternative approach. Let $\rho_{x}^{v}$ denote the "local density" of particles with velocity $v \in \mathscr{V}$. We first use the spectral gap and the logarithmic Sobolev inequality of the exclusion part of the dynamics, $\mathscr{L}^{e x}$, to reduce the local relevant parameters to $\rho_{x}^{v}, v \in \mathscr{V}$. On the other hand, only certain linear combinations of $\rho_{x}^{v}, v \in \mathscr{V}$ representing local mass and momentum are conserved by the collisions. We then use the collision part to eliminate the irrelevant modes. Details will be presented in Sects. 4 and 6. We also notice that the analysis of the asymmetric simple exclusion process presented in [8] contains an error which has been fixed in [15] using some estimates on the Green function of the simple exclusion process. Similar estimates are also needed in our setting here. We obtain such estimates using a method partly based on [15] and the extra difficulties due to collision are resolved in Sect. 4.

Finally we comment on the history of the methods used in this paper. The first mathematical rigorous proof of hydrodynamical limit for reversible nongradient models is the pioneering work of [16]. Though the Green-Kubo formula has widely been used to derive the hydrodynamic equation and certain mathematical rigor had been achieved before (see, for example, [17]), this work represents the first completely mathematical rigorous proof of the Green-Kubo formula and it gives a variational formula which is much more stable to work with. The key assumptions of this work are the reversibility of the process and the spectral gap of the dynamics. The spectral gap, though difficult, is more of a technical nature. It is proved in several models by $[18,19,20]$ and a general approach is outlined in [19]. A class of nonreversible models is treated by [21] but the final hydrodynamical equation is required to be a nonlinear heat equation without drift. The restriction on the drift is removed in [8] provided the space dimension $d>2$ and the logarithmic Sobolev inequality can be proved. A general argument to obtain the logarithmic Sobolev inequality is given in [22]. The restriction on the space dimension $d>2$ in [8] is optimal and one expects completely different behavior for $d \leqq 2$. In this case, the scaling is not diffusive and the hydrodynamical equation probably is nonlocal. There is no mathematical results concerning this phenomenon up to now. A similar phenomenon is expected also in the real hydrodynamics for $d \leqq 2$, due to the long time tails which cause the divergence of the transport coefficients [23].

We conclude with a few remarks on the viscosity matrix. The viscosity matrix we obtain does not reduce to the contribution due to the stochasticity of the model, corresponding to the symmetric part of the generator. Actually, we prove that the contribution due to the "deterministic motion," i.e. to the anti-symmetric part of the generator, is nonnegative and nonvanishing. For the real incompressible Navier-Stokes fluid all the eigenvalues coincide, but we cannot prove that this is true for Model II. We do not know if this is due to the lack of our analytic tools or due to the anisotropy of the underlying cubic lattice. Our analysis can be extended to lattices with other geometries, but we do not formulate an abstract framework for all possible lattices.

\section{The Models and the Main Theorem}

Let $\Lambda_{L} \in \mathbb{Z}^{d}$ be the cubic sublattice $\{-L, \ldots, L\}^{d}$ with periodic boundary conditions. Let $\varepsilon=L^{-1}$. We denote by $e_{i}, i=1, \ldots, d$, the coordinate vectors with component

$$
\left(e_{i}\right)_{j}=\delta_{i, j} \text {. }
$$


Moreover, we put $\mathscr{E}=\left\{e= \pm e_{i}\right.$ for some $\left.i=1, \ldots, d\right\} . R_{i} v$ will denote the reflection of the vector $v \in \mathbb{R}^{d}$ w.r.t. the plane $\pi_{i}$ orthogonal to $e_{i}$, i.e. the vector with coordinates

$$
\left(R_{i} v\right)_{j}= \begin{cases}-v_{i} & \text { if } i=j \\ v_{j} & \text { otherwise } .\end{cases}
$$

Denote by $\sigma$ any element of $\mathscr{J}$, the permutation group of the set $\{1, \ldots, d\}$, and let $\sigma v=\left(v_{\sigma(1)}, v_{\sigma(2)}, \ldots, v_{\sigma(d)}\right)$.

Let $\mathscr{V}$ be a finite subset of $\mathbb{R}^{d}$ representing the possible velocities and $N$ be its cardinality. Assume that $\mathscr{V}$ is symmetric w.r.t. reflections and permutations:

$$
R_{i} \mathscr{V}=\mathscr{V} \quad i=1, \ldots, d, \quad \sigma \mathscr{V}=\mathscr{V}, \quad \sigma \in \mathscr{J} .
$$

For our purposes it will be sufficient to consider velocities of fixed modulus, so we also assume

$$
|v|=\kappa \quad \text { for any } v \in \mathscr{V} .
$$

On each site of the lattice at most one particle for each velocity is allowed, with no exclusion rule between different velocities. A configuration of particles on the lattice is denoted by $\eta=\left\{\eta_{x}, x \in \Lambda_{L}\right\}$, where $\eta_{x}=\{\eta(x, v), v \in \mathscr{V}\}$ and $\eta(x, v)=0,1$, $x \in \mathbb{Z}^{d}, v \in V$ is the number of particles in $x$ with velocity $v$. The generator of the dynamics is defined by

$$
\mathscr{L} f=\mathscr{L}^{e x} f+\mathscr{L}^{c} f .
$$

$\mathscr{L}^{e x}$ is the generator of the exclusion process for particles with several colors (velocities), without exclusion between different colors

$$
\mathscr{L}^{e x} f(\eta)=\sum_{w \in \mathscr{V}} \sum_{\substack{y \in \mathbb{Z}^{d} \\ e \in \mathscr{E}}} p(y, y+e, w, \eta)\left[f\left(\eta^{y, y+e, w}\right)-f(\eta)\right],
$$

where

$$
\eta^{x, y, w}(z, v)= \begin{cases}\eta(z, v) & \text { if } v \neq w \text { or } z \neq x, y \\ \eta(x, w) & \text { if } v=w \text { and } z=y \\ \eta(y, w) & \text { if } v=w \text { and } z=x\end{cases}
$$

The jump intensity is given by

$$
\begin{gathered}
p(x, y, w, \eta)=\eta(x, w) p(x-y, w), \\
p(x-y, w)=\left[\chi+\frac{1}{2}(y-x) \cdot w\right], \quad v \in \mathscr{V},
\end{gathered}
$$

for some $\chi>\kappa / 2$. The constant $\chi$ has to be chosen large enough so that the jump rates are nonnegative.

The collision operator is defined as follows: let

$$
\mathscr{Q}=\left\{q=\left(v, w, v^{\prime}, w^{\prime}\right) \in \mathscr{V}^{4}: v+w=v^{\prime}+w^{\prime}\right\} .
$$

We call the quadruples $q \in \mathscr{Q}$ "collisions." $\mathscr{Q}$ is the set of all the possible collisions. The first two arguments of $q, v$ and $w$ are the incoming velocities, while $v^{\prime}, w^{\prime}$ are the outgoing velocities of the collision $q$. We define

$$
\mathscr{L}^{c} f(\eta)=\sum_{y \in \mathbb{Z}^{d}} \sum_{q \in \mathscr{Q}}\left[f\left(\eta^{y, q}\right)-f(\eta)\right]
$$


Here, for $q=\left(v, w, v^{\prime}, w^{\prime}\right)$ we put

$$
\tilde{p}(y, q, \eta)=\eta(y, v) \eta(y, w)\left(1-\eta\left(y, v^{\prime}\right)\right)\left(1-\eta\left(y, w^{\prime}\right)\right)
$$

and

$$
\eta^{y, q}(z, u)=\left\{\begin{array}{ll}
\eta(z, u) & \text { if } z \neq y \text { or } u \notin q \text { or } \tilde{p}(y, q, \eta)=0 \\
0 & \text { if } \tilde{p}(y, q, \eta)=1 \text { and } z=y \text { and }(u=v \text { or } u=w) \\
1 & \text { if } \tilde{p}(y, q, \eta)=1 \text { and } z=y \text { and }\left(u=v^{\prime} \text { or } u=w^{\prime}\right)
\end{array} .\right.
$$

Note that if $\eta$ does not contain in $y$ two particles with the incoming velocities $v$ and $w$, or there are particles with outgoing velocities $v^{\prime}$ and $w^{\prime}, \eta^{y, q}=\eta$. Putting $\left(Q_{y}^{q} f\right)(\eta) \equiv\left[f\left(\eta^{y, q}\right)-f(\eta)\right]$, we rewrite $(2.4)$ as

$$
\mathscr{L}^{c} f(\eta)=\sum_{y} \sum_{q \in \mathscr{Q}}\left(Q_{y}^{q} f\right)(\eta) .
$$

Specific choices of the set $\mathscr{V}$ will be given later on in this section. For the moment, we simply note that the collision operator conserves the total mass and the total momentum at every site. More precisely, the quantities

$$
I_{0}\left(\eta_{x}\right):=\sum_{v \in \mathscr{V}} \eta(x, v)
$$

and, for $\alpha=1, \ldots, d$ :

$$
I_{\alpha}\left(\eta_{x}\right):=\sum_{v \in \mathscr{V}}\left(v \cdot e_{\alpha}\right) \eta(x, v)
$$

are conserved in a collision, i.e., $\mathscr{L}^{c}\left[f\left(I_{\alpha}\left(\eta_{x}\right)\right)\right]=0$ for all $x \in \Lambda_{L}$ and for any function $f$. The choices of $\mathscr{V}$ will ensure that these are the only conserved quantities during a collision.

Since jumps conserve the total number of particles for each color, we have

$$
\mathscr{L}\left[\sum_{x} I_{\alpha}\left(\eta_{x}\right)\right]=0, \quad \alpha=0, \ldots, d .
$$

As a consequence, the following measure is invariant for $\mathscr{L}$

$$
d \mu_{L, r, n}=Z_{L, r, n}^{-1} \prod_{x \in \Lambda_{L}} \exp \left\{r I_{0}\left(\eta_{x}\right)+\sum_{\alpha=1}^{d} n_{\alpha} I_{\alpha}\left(\eta_{x}\right)\right\}
$$

with $n$ the $d$-dimensional vector $\left\{n_{\alpha}, \alpha=1, \ldots, d\right\}$. All the measures we will consider in the sequel are absolutely continuous w.r.t. the global equilibrium measure $\mu_{r}$ corresponding to $n=0$, namely the one defined as

$$
\mu_{r}(\eta)=Z_{r}^{-1} \prod_{x \in \Lambda_{L}} \exp \left\{r I_{0}\left(\eta_{x}\right)\right\}, \quad r \in \mathbb{R} .
$$

When possible we also omit the subscript $r$ and denote by $\langle\cdot\rangle$ the expectation w.r.t. $\mu_{r}$. 
The generator $\mathscr{L}$ is not symmetric w.r.t. this measure, because the generator of the exclusion part is not symmetric. However, one can check immediately that

$$
E^{\mu_{r}}\left[f \mathscr{L}^{c} g\right]=E^{\mu_{r}}\left[g \mathscr{L}^{c} f\right]
$$

so $\mathscr{L}^{c}$ is symmetric w.r.t $\mu_{r}$.

The formal adjoint w.r.t $\mu$ is given by

$$
\mathscr{L}^{*}=\left(\mathscr{L}^{e x}\right)^{*}+\mathscr{L}^{c}
$$

with

$$
\left(\mathscr{L}^{e x}\right)^{*} f(\eta)=\sum_{w \in \mathscr{V}} \sum_{\substack{y \in \mathbb{Z}^{d} \\ e \in \mathscr{E}}} p^{*}(y, y+e, w, \eta)\left[f\left(\eta^{y, y+e, w}\right)-f(\eta)\right]
$$

and

$$
p^{*}(x, y, w, \eta)=\eta(y, w)\left[\chi+\frac{1}{2}(y-x) \cdot w\right] .
$$

There are $d+1$ locally conserved quantities $I_{\alpha}(2.8)$ for this dynamics and the corresponding currents $w_{x, \beta}^{\alpha}, \alpha=0, \ldots, d$ are defined by

$$
\mathscr{L}\left[I_{\alpha}\left(\eta_{x}\right)\right]=\sum_{\beta=1}^{d} \nabla_{\beta}^{-} w_{x, \beta}^{\alpha}
$$

with

$$
\nabla_{\beta}^{-} g(x) \equiv g(x)-g\left(x-e_{\beta}\right), \quad \nabla_{\beta} g(x) \equiv g\left(x+e_{\beta}\right)-g(x) .
$$

Note that the collision operator plays no role in (2.13) because $\mathscr{L}^{c}\left[I_{\alpha}\left(\eta_{x}\right)\right]=0$. Similarly we define $w_{x, \beta}^{*, \alpha}$ as

$$
\mathscr{L}^{*}\left[I_{\alpha}\left(\eta_{x}\right)\right]=\sum_{\beta=1}^{d} \nabla_{\beta}^{-} w_{x, \beta}^{*, \alpha} .
$$

Since the current depends only on the exclusion part of the process and the exclusion dynamics is defined for each color separately, we can compute the currents using formulae for the asymmetric simple exclusion. More precisely, we can compute the current of the asymmetric simple exclusion process by

$$
\mathscr{L}^{e x} \eta(x, v)=\sum_{\beta=1}^{d} \nabla_{e}^{-} w_{x, \beta}(v), \quad \mathscr{L}^{*} \eta(x, v)=\sum_{\beta=1}^{d} \nabla_{\beta}^{-} w_{x, \beta}^{*}(v) .
$$

The currents $w_{x, \beta}(v)$ and $w_{x, \beta}^{*}(v)$ are given explicitly by

$$
\begin{aligned}
w_{x, \beta}(v) & =\frac{1}{2}\left(p\left(e_{\beta}, v\right)+p\left(-e_{\beta}, v\right)\right) \nabla_{\beta} \eta(x, v)+w_{x, \beta}^{(a)}(v), \\
w_{x, \beta}^{*}(v) & =\frac{1}{2}\left(p\left(e_{\beta}, v\right)+p\left(-e_{\beta}, v\right)\right) \nabla_{\beta} \eta(x, v)-w_{x, \beta}^{(a)}(v), \\
w_{x, \beta}^{(a)}(v) & =\left(p\left(e_{\beta}, v\right)-p\left(-e_{\beta}, v\right)\right) b_{x, \beta}(v),
\end{aligned}
$$

with

$$
b_{x, \beta}(v)=\eta\left(x+e_{\beta}, v\right) \eta(x, v)-\frac{1}{2}\left[\eta\left(x+e_{\beta}, v\right)+\eta(x, v)\right]
$$


Here $p\left(e_{\beta}, v\right)$ is the jump rate of the color $v$ in the direction $e_{\beta}$. With the convention (2.3), $p\left(e_{\beta}, v\right)-p\left(-e_{\beta}, v\right)=e_{\beta} \cdot v$ and $\frac{1}{2}\left(p\left(e_{\beta}, v\right)+p\left(-e_{\beta}, v\right)\right)=\chi$.

Using these equations, we obtain the mass current $w_{x, \beta}^{0}$ and the momentum current $w_{x, \beta}^{\alpha}$ as

$$
\begin{gathered}
w_{x, \beta}^{0}=\sum_{v \in \mathscr{V}} w_{x, \beta}(v)=\chi \nabla_{\beta} I_{0}\left(\eta_{x}\right)+\sum_{v \in \mathscr{V}}\left(e_{\beta} \cdot v\right) b_{x, \beta}(v), \\
w_{x, \beta}^{\alpha}=\sum_{v \in \mathscr{V}}\left(e_{\alpha} \cdot v\right) w_{x, \beta}(v)=\chi \nabla_{\beta} I_{\alpha}+\sum_{v \in \mathscr{V}}\left(e_{\alpha} \cdot v\right)\left(e_{\beta} \cdot v\right) b_{x, \beta}(v), \quad \alpha, \beta=1, \ldots, d .
\end{gathered}
$$

Similarly,

$$
\begin{array}{r}
w_{x, \beta}^{*, 0}=\chi \nabla_{\beta} I_{0}\left(\eta_{x}\right)-\sum_{v \in \mathscr{V}}\left(e_{\beta} \cdot v\right) b_{x, \beta}(v), \\
w_{x, \beta}^{*, \alpha}=\chi \nabla_{\beta} I_{\alpha}-\sum_{v \in \mathscr{V}}\left(e_{\alpha} \cdot v\right)\left(e_{\beta} \cdot v\right) b_{x, \beta}(v), \quad \alpha, \beta=1, \ldots, d .
\end{array}
$$

It is convenient to introduce also the symmetric and antisymmetric part of the currents

$$
w_{x, \beta}^{(s), \alpha}=\frac{w_{x, \beta}^{\alpha}+w_{x, \beta}^{*, \alpha}}{2}, \quad w_{x, \beta}^{(a), \alpha}=\frac{w_{x, \beta}^{\alpha}-w_{x, \beta}^{*, \alpha}}{2} .
$$

We will need to compute averages w.r.t. distributions of the form

$$
\mu_{n, L}=Z^{-1} \prod_{x \in \Lambda_{L}} \exp \left\{n_{0}(x) I_{0}\left(\eta_{x}\right)+\sum_{\alpha=1}^{d} n_{\alpha}(x) I_{\alpha}\left(\eta_{x}\right)\right\} .
$$

Define the velocity distribution at $x$ as

$$
f(x, v, n)=E^{\mu_{n}}[\eta(x, v)]=\frac{e^{n_{0}(x)+\underline{n}(x) \cdot v}}{1+e^{n_{0}(x)+\underline{n}(x) \cdot v}},
$$

with $\underline{n}=\left(n_{1}, \ldots, n_{d}\right)$. The average of the currents $w_{x, \beta}^{\alpha}$ w.r.t. a measure of this form are given by

$$
\begin{aligned}
E^{\mu_{n}}\left[w_{x, \beta}^{0}\right]= & \sum_{v \in \mathscr{V}}\left(e_{\beta} \cdot v\right) E^{\mu_{n}}\left[b_{x, \beta}(v)\right]+\chi \nabla_{\beta} E^{\mu_{n}}\left[I_{0}\right] \\
= & \sum_{v \in \mathscr{V}}\left(v \cdot e_{\beta}\right)\left[f(x, v, n) f\left(x+e_{\beta}, v, n\right)-\frac{1}{2}\left(f(x, v, n)+f\left(x+e_{\beta}, v, n\right)\right)\right] \\
& +\chi \nabla_{\beta} E^{\mu_{n}}\left[I_{0}\right] \\
E^{\mu_{n}}\left[w_{x, \beta}^{\alpha}\right]= & \sum_{v \in \mathscr{V}}\left(e_{\alpha} \cdot v\right)\left(e_{\beta} \cdot v\right) E^{\mu_{n}}\left[b_{x, \beta}(v)\right]+\chi \nabla_{\beta} E^{\mu_{n}}\left[I_{\alpha}\right] \\
= & \sum_{v \in \mathscr{V}}\left(e_{\alpha} \cdot v\right)\left(e_{\beta} \cdot v\right)\left[f(x, v, n) f\left(x+e_{\beta}, v, n\right)-\frac{1}{2}(f(x, v, n)\right. \\
& \left.+f\left(x+e_{\beta}, v, n\right)\right]+\chi \nabla_{\beta} E^{\mu_{n}}\left[I_{\alpha}\right] .
\end{aligned}
$$

Let us give now a heuristic derivation of the hydrodynamic equations.

We start with the Euler scale. We choose as initial state for the system the local equilibrium measure $(2.20)$ with chemical potentials $\tilde{n}(\varepsilon x, 0)$ slowly varying 
in space. Then we look at the system on the Euler time scale, i.e. at times of order $\varepsilon^{-1}$, that corresponds to replace the generator $\mathscr{L}$ with $\varepsilon^{-1} \mathscr{L}$. Then the hydrodynamic equations are recovered from the conservation laws

$$
\frac{d}{d t} \varepsilon^{d} \sum_{x} J(\varepsilon x) E^{f_{t} \mu_{r}}\left[I_{\alpha}\left(\eta_{x}\right)\right]=\varepsilon^{d-1} \sum_{x} \sum_{\beta=1}^{d} J(\varepsilon x) \nabla_{\beta}^{-} E^{f_{t} \mu_{r}}\left[w_{x, \beta}^{\alpha}\right],
$$

where $f_{t} \mu_{r}$ denotes the distribution of the process at time $t$.

Assuming that $f_{t}$ is still well approximated by a local equilibrium with chemical potentials $\tilde{n}(\varepsilon x, t)$, by $(2.21)$ and $(2.22)$, in the limit $\varepsilon \rightarrow 0$ we get the Euler equations

$$
\frac{\partial}{\partial t} \rho+\sum_{\alpha=1}^{d} \partial_{z_{\alpha}} j_{a}=0, \quad \frac{\partial}{\partial t} u_{\alpha}+\sum_{\beta=1}^{d} \partial_{z_{\beta}} \pi^{\alpha \beta}=0,
$$

where $\partial_{z_{\alpha}}$ denotes the partial derivative w.r.t. the macroscopic coordinate $z_{\alpha}$,

$$
\rho(\varepsilon x, t)=E^{\mu_{\tilde{n}}}\left[I_{0}\left(\eta_{x}\right)\right]=\sum_{v \in \mathscr{V}} f(x, v, \tilde{n})
$$

is the mass density,

$$
\bar{u}_{\alpha}(\varepsilon x, t)=E^{\mu_{\tilde{n}}}\left[I_{\alpha}\left(\eta_{x}\right)\right]=\sum_{v \in \mathscr{V}}\left(v \cdot e_{\alpha}\right) f(x, v, \tilde{n})
$$

is the momentum density,

$$
j_{\alpha}(\varepsilon x, t)=\sum_{v \in \mathscr{V}}\left(v \cdot e_{\alpha}\right)\left(f(x, v, \tilde{n})-f(x, v, \tilde{n})^{2}\right)
$$

is the mass current and

$$
\begin{aligned}
\pi^{\alpha \beta}(\varepsilon x, t) & =E^{\mu_{\tilde{n}}}\left[\left(I_{\alpha}\left(\eta_{x}\right)-E^{\mu_{\tilde{n}}}\left[I_{\alpha}\left(\eta_{x}\right)\right]\right)\left(I_{\beta}\left(\eta_{x}\right)-E^{\mu_{\tilde{n}}}\left[I_{\beta}\left(\eta_{x}\right)\right]\right)\right] \\
& =\sum_{v \in \mathscr{V}}\left(e_{\alpha} \cdot v\right)\left(e_{\beta} \cdot v\right)\left[f(x, v, \tilde{n})-f(x, v, \tilde{n})^{2}\right]
\end{aligned}
$$

is the stress tensor.

A more explicit expression for $\rho, \bar{u}_{\alpha}, j_{\alpha}$ and $\pi^{\alpha \beta}$ is obtained from the symmetry of $\mathscr{V}$ in the exchange $v \rightarrow-v$. We have:

$$
\begin{aligned}
\rho & =\sum_{v \in \mathscr{V}} \frac{\mathrm{e}^{2 \tilde{n}_{0}}+\mathrm{e}^{\tilde{n}_{0}} \cosh (\tilde{n} \cdot v)}{1+\mathrm{e}^{2 \tilde{n}_{0}}+2 \mathrm{e}^{\tilde{n}_{0}} \cosh (\tilde{n} \cdot v)}, \\
\bar{u}_{\alpha} & =\sum_{v \in \mathscr{V}}\left(v \cdot e_{\alpha}\right) \frac{\mathrm{e}^{\tilde{n}_{0}} \sinh (\tilde{n} \cdot v)}{1+\mathrm{e}^{2 \tilde{n}_{0}}+2 \mathrm{e}^{\tilde{n}_{0}} \cosh (\tilde{n} \cdot v)}, \\
j_{\alpha} & =\sum_{v \in \mathscr{V}}\left(v \cdot e_{\alpha}\right) \frac{\mathrm{e}^{\tilde{n}_{0}} \sinh (\tilde{n} \cdot v)\left(1-\mathrm{e}^{2 \tilde{n}_{0}}\right)}{\left(1+\mathrm{e}^{2 \tilde{n}_{0}}+2 \mathrm{e}^{\tilde{n}_{0}} \cosh (\tilde{n} \cdot v)\right)^{2}}, \\
\pi^{\alpha \beta} & =\sum_{v \in \mathscr{V}}\left(v \cdot e_{\alpha}\right)\left(v \cdot e_{\beta}\right) \frac{2 \mathrm{e}^{\tilde{n_{0}}} \cosh (\tilde{n} \cdot v)\left(1+\mathrm{e}^{2 \tilde{n}_{0}}\right)+4 \mathrm{e}^{2 \tilde{n}_{0}}}{\left(1+\mathrm{e}^{2 \tilde{n_{0}}}+2 \mathrm{e}^{\tilde{n}_{0}} \cosh (\tilde{n} \cdot v)\right)^{2}} .
\end{aligned}
$$

Next, we consider the incompressible limit; we follow the same strategy. We choose as initial state for the system the local equilibrium measure (2.20) with 
chemical potentials $\tilde{n}(\varepsilon x, 0)$ given by

$$
\tilde{n}_{0}=r+\varepsilon^{2} \lambda_{0}(\varepsilon x), \quad \tilde{n}_{\alpha}=\varepsilon \lambda_{\alpha}(\varepsilon x),
$$

such that

$$
\operatorname{div} \lambda:=\sum_{\alpha=1}^{d} \partial_{z_{\alpha}} \lambda_{\alpha}=0
$$

The conservation laws are the same as (2.22) except a change of scaling is needed, i.e. we replace $\mathscr{L}$ by $\varepsilon^{-2} \mathscr{L}$ :

$$
\frac{\partial}{\partial t} \varepsilon^{d} \sum_{x} J(\varepsilon x) E^{f_{l} \mu_{r}}\left[I_{\alpha}\left(\eta_{x}\right)\right]=\varepsilon^{d-2} \sum_{x} \sum_{\beta=1}^{d} J(\varepsilon x) \nabla_{\beta}^{-} E^{f_{l} \mu_{r}}\left[w_{x, \beta}^{\alpha}\right] .
$$

Assume that a local equilibrium measure with chemical potentials like in (2.27) describes the system also at time $t$. With this choice of the local equilibrium, we have

$$
\begin{gathered}
\rho=N \theta+O\left(\varepsilon^{2}\right), \quad \theta=\frac{e^{r}}{1+e^{r}}, \\
E^{\mu_{\tilde{n}}}[\eta(x, v)+\eta(x,-v)]=2 \theta+O\left(\varepsilon^{2}\right) .
\end{gathered}
$$

Moreover, the assumption on $\mathscr{V}$ imply that

$$
\begin{gathered}
\sum_{v \in \mathscr{V}}\left(e_{\alpha} \cdot v\right)\left(e_{\beta} \cdot v\right)=K \delta_{\alpha, \beta}, \\
\sum_{v \in \mathscr{V}}\left(e_{\alpha} \cdot v\right)\left(e_{\beta} \cdot v\right)(\lambda \cdot v)^{2}=t \delta_{\alpha, \beta} A|\lambda|^{2}+\delta_{\alpha, \beta} B \lambda_{\alpha}^{2}+C \lambda_{\alpha} \lambda_{\beta},
\end{gathered}
$$

where $A, B, C$ and $K$ are constants independent of $\lambda$.

Therefore,

$$
\bar{u}_{\alpha}=\varepsilon t(\theta) \lambda_{\alpha}+O\left(\varepsilon^{3}\right),
$$

with

$$
t(\theta)=K \theta(1-\theta) .
$$

We have used the following identities:

$$
\begin{aligned}
& \frac{\exp \left[r+\varepsilon b+\varepsilon^{2} c\right]}{1+\exp \left[r+\varepsilon b+\varepsilon^{2} c\right]}=\theta+\varepsilon \theta(1-\theta) b+\frac{\varepsilon^{2}}{2} \theta(1-\theta)\left[2 c+(1-2 \theta) b^{2}\right]+O\left(\varepsilon^{3}\right), \\
& \quad \frac{\exp \left[r+\varepsilon b+\varepsilon^{2} c\right]}{1+\exp \left[r+\varepsilon b+\varepsilon^{2} c\right]}-\left\{\frac{\exp \left[r+\varepsilon b+\varepsilon^{2} c\right]}{1+\exp \left[r+\varepsilon b+\varepsilon^{2} c\right]}\right\}^{2} \\
& \quad=\theta(1-\theta)+\varepsilon \theta(1-\theta)(1-2 \theta) b+\frac{\varepsilon^{2}}{2} \theta(1-\theta)[2(1-2 \theta) c \\
& \left.\quad+(1-6(1-\theta) \theta) b^{2}\right]+O\left(\varepsilon^{3}\right),
\end{aligned}
$$

where $\theta=e^{r} /\left(1+e^{r}\right)$. From this identities and (2.27),

$$
\begin{aligned}
& f(x, v, n)=\theta+\varepsilon \theta(1-\theta)(\lambda \cdot v)+\frac{\varepsilon^{2}}{2} \theta(1-\theta)\left[2 \lambda_{0}+(1-2 \theta)(\lambda \cdot v)^{2}\right]+O\left(\varepsilon^{3}\right), \\
& f(x, v, n)^{2}-f(x, v, n)=-\theta(1-\theta)-\varepsilon \theta(1-\theta)(1-2 \theta)(\lambda \cdot v) \\
& -\frac{\varepsilon^{2}}{2} \theta(1-\theta)\left[2(1-2 \theta) \lambda_{0}+(1-6(1-\theta) \theta)(\lambda \cdot v)^{2}\right]+O\left(\varepsilon^{3}\right) .
\end{aligned}
$$


Equations (2.29) $-(2.31)$ are direct consequences of the last identities.

From (2.21) and (2.34) one can check that

$$
\begin{aligned}
\varepsilon^{d-2} & \sum_{x} \sum_{\beta=1}^{d} \nabla_{\beta}^{-} E^{\mu \tilde{n}}\left[w_{x \beta}^{0}\right] J(\varepsilon x) \\
= & \varepsilon^{d} \sum_{x} \sum_{\beta=1}^{d} J(\varepsilon x)\left\{(2 \theta-1) t(\theta) \partial_{z_{\beta}} \lambda_{\beta}+\chi \partial_{z_{\beta} z_{\beta}}^{2} \rho\right\}+O\left(\varepsilon^{2}\right),
\end{aligned}
$$

because

$$
\nabla_{\beta}^{-}\left[\lambda\left(\varepsilon\left(x+e_{\beta}\right)\right)+\lambda(\varepsilon x)\right]=2 \varepsilon \partial_{z_{\beta}} \lambda(\varepsilon x)+O\left(\varepsilon^{3}\right) .
$$

From (2.29) the average of the 1.h.s. of (2.28) with $\alpha=0$ is $O\left(\varepsilon^{2}\right)$. Moreover, the last term on the right-hand side of (2.35) is of order $\varepsilon^{2}$. Hence we have to assume $\operatorname{div} \lambda=0$ to assure the continuity equation. Also, the leading order correction to $(2.35)$ is of order $\varepsilon^{2}$, hence it is consistent with the assumption that the density correction is of order $\varepsilon^{2}(2.27)$. We remark that, if there is a first order correction to the density, which can be achieved by adding a term $\varepsilon \lambda_{1}(\varepsilon x, t)$ in the first equation of (2.27), then the leading contribution to the last term in $(2.35)$ is

$$
\chi \varepsilon^{d+1} \sum_{x} \sum_{\beta=1}^{d} \partial_{z_{\beta} z_{\beta}}^{2} J(\varepsilon x) \rho_{1},
$$

where $\rho_{1}$ is the corresponding first order correction to the density (i.e., $\rho=\rho_{0}+\varepsilon \rho_{1}$ ). Note that this term appears because of the stochastic nature of our dynamics (more precisely, because we use asymmetric simple exclusions to replace the deterministic dynamics).

The averages of the momentum currents in (2.28) can be computed as

$$
\begin{aligned}
& \varepsilon^{-3} \sum_{\beta=1}^{d} E^{\mu_{\tilde{n}}} \nabla_{\beta}^{-}\left[w_{x \beta}^{\alpha}\right]=-t(\theta)(1-2 \theta) \sum_{\beta=1}^{d} \partial_{z_{\alpha}} \lambda_{0}+\chi t(\theta) \sum_{\beta=1}^{d} \partial_{z_{\beta} z_{\beta}}^{2} \lambda_{\alpha} \\
& -\frac{\theta}{2}(1-\theta)(1-6 \theta(1-\theta)) \sum_{v \in \mathscr{V}} \sum_{\beta=1}^{d}\left(e_{\alpha} \cdot v\right)\left(e_{\beta} \cdot v\right) \partial_{z_{\beta}}(\lambda \cdot v)^{2}+O(\varepsilon)
\end{aligned}
$$

Recall that $\mathscr{V}$ is invariant under the reflection w.r.t each coordinate axis. Hence, by (2.36), the equations for $\lambda_{\alpha}$ are of the form

$$
\frac{\partial}{\partial t} \lambda_{\alpha}=-\frac{1}{t(\theta)} \partial_{z_{\alpha}} p-\frac{B h(\theta)}{t(\theta)} \partial_{z_{\alpha}} \lambda_{\alpha}^{2}-\frac{C h(\theta)}{t(\theta)} \sum_{\beta=1}^{d} \lambda_{\beta} \partial_{z_{\beta}} \lambda_{\alpha}+\chi \sum_{\beta=1}^{d} \partial_{z_{\beta} z_{\beta}}^{2} \lambda_{\alpha},
$$

where

$$
p(\theta, \lambda)=t(\theta)(1-2 \theta) \lambda_{0}+A h(\theta)|\lambda|^{2}, \quad h(\theta)=\frac{\theta}{2}(1-\theta)(1-6 \theta(1-\theta)) .
$$

We obtain the usual Navier-Stokes equation (up to a scale factor) if the coefficient $B=0$. An example of $\mathscr{V}$ such that this is true will be given in Model II below. 
This heuristic derivation is not entirely correct. The diffusion coefficient appearing in (2.37) comes completely from the stochastic fluctuation, namely, the fluctuation associated with the simple exclusion process. Therefore, if (2.37) were correct, there would be no contribution to the viscosity from the "deterministic part" of the dynamics. We shall prove that (2.37) still holds but the diffusion coefficient is not given by $\chi$. The true diffusion coefficient is always bigger than $\chi$ and this accounts for the viscosity from the "deterministic part" of the dynamics.

We now choose the space $\mathscr{V}$. First notice that, because of the symmetry properties of $\mathscr{V}$, the constants $A, B, C$ and $D$ have the more explicit expressions

$$
A=\sum_{v \in \mathscr{V}} v_{1}^{2} v_{2}^{2}=C / 2, \quad B=\sum_{v \in \mathscr{V}}\left[v_{1}^{4}-3 v_{1}^{2} v_{2}^{2}\right], \quad K=\sum_{v \in \mathscr{V}} v_{1}^{2} .
$$

Model I. The simplest choice is the following: Let

$$
\mathscr{V}=\mathscr{E} .
$$

With this choice, the only possible collisions are those $q=\left(v, w, v, w^{\prime}\right)$ such that $v+w=0$ and $v^{\prime}+w^{\prime}=0$.

For this model, one can easily check that

$$
A=C=0, \quad B=2, \quad K=2 \text {. }
$$

Model II. Let $d=3$ and $\sigma$ denote any element of $\mathscr{J}$, the permutation group of $\{1,2,3\}$. Let

$$
\mathscr{V}=\{v: \sigma v=( \pm 1, \pm 1, \pm \varpi) \text { for some } \sigma \in \mathscr{J}\},
$$

where $\varpi$ is the positive solution of

$$
\varpi^{4}-6 \varpi^{2}-1=0
$$

$\mathscr{V}$ is invariant under reflections w.r.t. any coordinate plane and permutations of coordinate axes. Moreover, with the above choice of $\varpi$, one gets:

$$
B=0
$$

and

$$
A=8\left(1+2 \varpi^{2}\right), \quad C=16\left(1+2 \varpi^{2}\right), \quad K=8\left(2+\varpi^{2}\right) .
$$

The proof that the only collision invariants are total mass and total momentum is part of the ergodic theorem proven in next section.

Now we are ready to state our convergence result. Let $f_{t}$ be the distribution density, w.r.t. $\mu_{r}$, of the process on the diffusive time scale, namely the solution of the forward equation

$$
\frac{\partial}{\partial t} f_{t}=\varepsilon^{-2} \mathscr{L}^{*} f_{t}
$$

We consider the initial datum $f_{t=0}=\psi_{0}$ given by

$$
\psi_{0}=Z_{\varepsilon}^{-1} \exp \left[\varepsilon \sum_{x \in \Lambda_{L}} \sum_{\alpha=1}^{d} \lambda_{\alpha}(\varepsilon x) I_{\alpha}(x)\right]
$$


with $\lambda_{\alpha}$ smooth periodic functions. Recall the definition of the specific relative entropy of two densities $f$ and $g$ w.r.t. $\mu_{r}$ :

$$
s(f \mid g)=\varepsilon^{d}\left\langle f \log \frac{f}{g}\right\rangle
$$

and let

$$
s(f) \equiv s(f \mid 1)
$$

The rest of this paper is devoted to prove the following

Theorem 2.1. Assume $d \geqq 3$ and $\mathscr{V}$ as in Model I or Model II. Let $f_{t}$ the density w.r.t. the measure $\mu_{r}$, defined in (2.10), at time $t$. Then there is a matrix $D$ with coefficients $D_{\beta, \gamma}^{\alpha, v}, \alpha, \beta, \gamma, v=1, \ldots, d$, such that $D \geqq D_{s}=\chi \mathbb{I}$ (as matrices) and the following holds: given a smooth solution $p(x, t), u(x, t)$ of the incompressible Navier-Stokes equations

$$
\begin{aligned}
\operatorname{div} u & =0 \\
\frac{\partial}{\partial t} u_{\alpha}+\frac{h(\theta)}{t(\theta)^{2}}\left[B \partial_{z_{\alpha}} u_{\alpha}^{2}+C \sum_{\beta=1}^{d} u_{\beta} \partial_{z_{\beta}} u_{\alpha}\right] & =-\partial_{z_{\alpha}} p+\sum_{\beta, \gamma, v=1}^{d} D_{\beta, \gamma}^{\alpha, v} \partial_{z_{\beta}} \partial_{z_{\gamma}} u_{v},
\end{aligned}
$$

we define the following density $\Psi_{t}$ w.r.t. $\mu_{r}$,

$$
\Psi_{t}=Z_{t}^{-1} \exp \left[\varepsilon \sum_{x \in \Lambda_{L}} \sum_{\alpha=1}^{d} \lambda_{\alpha}(\varepsilon x, t) I_{\alpha}(x)\right], \quad \lambda_{\alpha}=t(\theta)^{-1} u_{\alpha}
$$

Then:

1)

$$
\lim _{\varepsilon \rightarrow 0} \varepsilon^{-2} s\left(f_{t} \mid \Psi_{t}\right)=0
$$

2) Defining the empirical fields $v_{\alpha}^{\varepsilon}$ for $\alpha=0, \ldots, d$, as

$$
\begin{gathered}
v_{0}^{\varepsilon}(x, t)=\varepsilon^{d} \sum_{x \in \Lambda_{L}} \delta(z-\varepsilon x) I_{0}\left(\eta_{x}(t)\right), \\
v_{\alpha}^{\varepsilon}(x, t)=\varepsilon^{d-1} \sum_{x \in \Lambda_{L}} \delta(z-\varepsilon x) I_{\alpha}\left(\eta_{x}(t)\right), \quad \alpha=1, \ldots, d,
\end{gathered}
$$

we have that, for $\alpha=1, \ldots, d, v_{\alpha}^{\varepsilon}$ converges weakly in probability to $u_{\alpha}$, solution of the Navier-Stokes equation, and $v_{0}^{\varepsilon}$ converges to the constant $N \theta$.

3) The matrix $D \geqq D_{s}$ is diagonal and has the form

$$
D_{\beta, \gamma}^{\alpha, v}=\delta_{\beta, \gamma} \delta_{\alpha, v}\left[D_{1}+D_{2} \delta_{\alpha, \beta}\right]+D_{3} \delta_{\alpha, \beta} \delta_{v, \gamma}+D_{4} \delta_{\alpha, \gamma} \delta_{\beta, v} .
$$

Moreover

$$
D \neq D_{s} .
$$


The proof of this theorem is given in Sect. 3 and it is based on the lemmas and theorems of Sects. 4-6.

Remarks.

(i) The matrix $D_{s}$ is the diffusion matrix which appears in the limiting equations when one considers only the symmetric part of the generator $\mathscr{L}_{s}=1 / 2\left(\mathscr{L}+\mathscr{L}^{*}\right)$. The true diffusion matrix $D$ is bigger than $D_{s}$, as stated in the last part of Theorem 2.1 because of the contribution of the deterministic motion.

(ii) The second part of the theorem is a consequence of the relative entropy estimate by means of the following entropy inequality. If $f$ and $g$ are normalized densities w.r.t. $d \mu$, then

$$
E^{f}[X] \leqq \gamma^{-1} \varepsilon^{-d} s(f \mid g)+\gamma^{-1} \log E^{g}[\exp \{\gamma X\}]
$$

for any positive $\gamma$ and for any random variable $X$. See $[24,10]$ and Lemma 2.2 below for details.

(iii) The factor $t(\theta)$ is due to the relation between the momentum density $\bar{u}$ and its conjugate chemical potential $\lambda$ at the first order as in (2.31). Here $\bar{u}=\varepsilon u+O\left(\varepsilon^{2}\right)$.

(iv) We need the specific form of $\mathscr{V}$ only in the proof of the ergodic theorem, Theorem 3.3 in the next section. The rest of the proof is valid under quite general conditions. Therefore Theorem 2.1 holds for any model in $d \geqq 3$, with $\mathscr{V}$ a finite set of vectors of given length, invariant under reflections and permutation of axes, for which the ergodic Theorem 3.3 is valid.

To conclude, we recall a lemma from [8] (Lemma 2.2 of [8], p. 1239).

Lemma 2.2. Let $f$ be a density satisfying

$$
\lim _{\varepsilon \rightarrow 0} \varepsilon^{-2} s(f \mid \psi)=0
$$

where $\psi$ is a density of a product measure of the type (2.46). Then for any $J$ and any bounded local function $F$ one has

$$
\lim _{\varepsilon \rightarrow 0}\left[E^{f}-E^{\psi}\right]\left[\varepsilon^{d-1} \sum_{x} J(\varepsilon x) \tau_{x} F\right]=0 .
$$

If instead of $\varepsilon^{-2} s(f \mid \psi) \rightarrow 0$ one has $\varepsilon^{-2} s(f \mid \psi) \leqq$ const., then the following bound holds:

$$
\left|\left[E^{f}-E^{\psi}\right]\left[\varepsilon^{d-1} \sum_{x} J(\varepsilon x) \tau_{x} F\right]\right| \leqq \text { const. }
$$

In particular, $\psi$ can be the equilibrium measure $\mu_{r}$.

Finally, a direct computation shows that

$$
s(\psi)=\varepsilon^{d} E^{\mu_{r}}[\psi \log \psi] \leqq \text { const. } \varepsilon^{2},
$$

so that the previous lemma assures that $\left|\left[E^{\psi}-E^{\mu_{r}}\right]\left[\varepsilon^{d-1} \sum_{x} J(\varepsilon x) \tau_{x} F\right]\right| \leqq$ const. 


\section{The Relative Entropy Estimate}

The strategy of the proof of Theorem 2.1 is similar to the one used in [8]. The first step is to choose a local equilibrium density, $\tilde{\Psi}_{t}$, (and suitable corrections) to compare to the density $f_{t}$ of the actual time evolution. This density will be chosen in such a way that its parameters to the first order satisfy the hydrodynamic equations, while the second order terms take into account the contribution of the fast modes. More precisely, we put

$$
\begin{aligned}
\tilde{\Psi}_{t}= & \tilde{Z}_{t}^{-1} \exp \left[\varepsilon \sum_{x \in \Lambda_{L}} \sum_{\alpha=1}^{d}\left(\lambda_{\alpha} * \hat{\omega}\right)(\varepsilon x, t) I_{\alpha}\left(\eta_{x}\right)\right. \\
& \left.+\varepsilon^{2}\left(\sum_{x \in \Lambda_{L}}\left(\lambda_{0} * \hat{\omega}\right)(\varepsilon x, t) I_{0}\left(\eta_{x}\right)+\Phi(\eta)\right)\right] .
\end{aligned}
$$

Here $\tilde{Z}_{t}$ is the normalization, $*$ is the convolution product on $\mathbb{Z}^{d}$, and $\lambda_{\alpha}(z, t), \hat{\omega}$ and $\Phi$ are chosen as follows:

(3.A) Let $\ell$ and $k=\ell \varepsilon^{-2 / d}$ be integers, $\bar{\ell}=\ell^{d+2}$ and suppose that $\Lambda_{k}$ is divided in disjoint cubes of size $(2 \bar{\ell}+1)$, with centers $\sigma \in(2 \bar{\ell}+1) \mathbb{Z}^{d},|\sigma| \leqq k$. Let $\bar{\ell}_{1}=\bar{\ell}-\ell^{1 / d^{2}}$ and consider the cubes $\Lambda_{\bar{\ell}_{1}, \sigma}$ and $\tilde{\Lambda}_{k}=\bigcup_{|\sigma| \leqq k} \Lambda_{\bar{\ell}_{1}, \sigma}$ is the region $\Lambda_{k}$ without corridors of width $2 \ell^{1 / d^{2}}$. Define $\hat{\omega}$ and $\omega$ to be the normalized characteristic functions

$$
\hat{\omega}(x)=\left|\tilde{\Lambda}_{k}\right|^{-1} \mathbb{1}\left(x \in \tilde{\Lambda}_{k}\right), \quad \omega(x)=(2 k+1)^{-d} \mathbb{1}\left(x \in \Lambda_{k}\right) .
$$

(3.B) The chemical potential $\lambda_{\alpha}, \alpha=1, \ldots, d$, are solutions of

$$
\begin{gathered}
\operatorname{div} \lambda \equiv \sum_{\alpha=1}^{d} \partial_{z_{\alpha}} \lambda_{\alpha}=0 \\
\frac{\partial}{\partial t} \lambda_{\alpha}=-\frac{1}{t(\theta)} \partial_{z_{\alpha}} p-\frac{h(\theta)}{t(\theta)}\left\{B \partial_{z_{\alpha}} \lambda_{\alpha}^{2}+C \sum_{\beta=1}^{d} \lambda_{\beta} \partial_{z_{\beta}} \lambda_{\alpha}\right\}+\sum_{\beta, \gamma=1}^{d} D_{\beta \gamma}^{\alpha} \partial_{z_{\beta} z_{\gamma}}^{2} \lambda_{\alpha},
\end{gathered}
$$

with $D_{\beta \gamma}^{\alpha}$ given as in Theorem 2.1,

$$
\begin{gathered}
p\left(\theta, \lambda_{0}, \lambda_{\alpha}\right)=t(\theta)(1-2 \theta) \lambda_{0}+A h(\theta) \lambda^{2}, \\
t(\theta)=K \theta(1-\theta), \quad h(\theta)=\frac{\theta}{2}(1-\theta)(1-6 \theta(1-\theta)) .
\end{gathered}
$$

(3.C) Choice of $\Phi: \Phi(\eta)=-\sum_{x \in \Lambda_{L}} \sum_{\alpha, \beta=1}^{d} \partial_{\alpha} \lambda_{\beta}(\varepsilon x, t)\left(\hat{\omega} * \tau_{y} F_{\alpha}^{\beta}\right)$ where $F_{\alpha}^{\beta}$ are local functions satisfying the conditions in Definition 4.1 of the next section.

The choice of $\hat{\omega}$ is for the convenience that no boundary terms arise in the multiscale analysis; one can simply use $\omega$ but a few steps are needed to bound the boundary terms. The multi-scale analysis is an important tool in proving Theorem 4.6 of [8]. Its extension to the present setup will be used extensively here. 
For the convenience of later reference, recall for our models the currents are given by

$$
w_{x, \beta}^{*, \alpha}=w_{x, \beta}^{(s) \alpha}-w_{x, \beta}^{(a) \alpha}, \quad w_{x, \beta}^{(s) \alpha}=\chi \nabla_{\beta} I_{\alpha}
$$

and

$$
\begin{aligned}
& w_{x, \beta}^{(a) \alpha}=\sum_{v \in \mathscr{V}}\left(e_{\alpha} \cdot v\right)\left(e_{\beta} \cdot v\right) b_{x, \beta}(v), \\
& w_{x, \beta}^{(a) 0}=\sum_{v \in \mathscr{V}_{+}}\left(e_{\beta} \cdot v\right) b_{x, \beta}(v),
\end{aligned}
$$

where

$$
b_{x, \beta}(v)=\eta\left(x+e_{\beta}, v\right) \eta(x, v)-\frac{1}{2}\left[\eta\left(x+e_{\beta}, v\right)+\eta(x, v)\right] .
$$

Furthermore, the density, velocity, stress tensor and the pressure are related by

$$
\begin{array}{r}
\rho=N \theta+O\left(\varepsilon^{2}\right), \quad \theta=\frac{e^{r}}{1+e^{r}}, \quad u_{\alpha}=\varepsilon t(\theta) \lambda_{\alpha}+O\left(\varepsilon^{3}\right), \\
\pi^{\alpha \beta}=\left(t(\theta)+\varepsilon^{2} p(\theta, \lambda)\right) \delta_{\alpha, \beta}+\varepsilon^{2} h(\theta)\left(B \lambda_{\alpha}^{2} \delta_{\alpha, \beta}+C \lambda_{\alpha} \lambda_{\beta}\right) .
\end{array}
$$

We return to the proof of Theorem 2.1. The density $\tilde{\Psi}_{t}$ differ from $\Psi_{t}$ only at the second order in $\varepsilon$. The following lemma shows that the difference of $s(f \mid \Psi)$ and $s(f \mid \tilde{\Psi})$ is small.

Lemma 3.1. Suppose that $f$ is a probability measure on $\Lambda_{L}$. Let

$$
\begin{gathered}
\phi=Z^{-1} \exp \left[\sum_{x \in \Lambda_{L}} \sum_{\alpha=1}^{d} \varepsilon S_{\alpha}(\varepsilon x, t) I_{\alpha}\left(\eta_{x}\right)\right], \\
\phi^{\prime}=Z^{\prime-1} \exp \left[\sum_{x \in \Lambda_{L}} \sum_{\alpha=1}^{d}\left(\varepsilon S_{\alpha}(\varepsilon x, t) I_{\alpha}\left(\eta_{x}\right)+\varepsilon^{2} t_{\alpha}(\varepsilon x, t) \tau_{x} F_{\alpha}\right)\right],
\end{gathered}
$$

two densities w.r.t. $\mu_{r}$ where $S_{\alpha}$ and $t_{\alpha}$ are smooth functions of $(z, t)$ and $F_{\alpha}$ local functions of the configuration such that $E\left[F_{\alpha}\right]=0$. If $\varepsilon^{-2} s(f \mid \phi) \rightarrow 0$, then

$$
\lim _{\varepsilon \rightarrow 0} \varepsilon^{-2}\left[s(f \mid \phi)-s\left(f \mid \phi^{\prime}\right)\right]=0 .
$$

The proof is an obvious extension of the one given in [8].

From this remark, to prove Theorem 2.1 we shall only prove

$$
\lim _{\varepsilon \rightarrow 0} \varepsilon^{-2} s\left(f_{t} \mid \tilde{\Psi}_{t}\right)=0 \text {. }
$$

To state the main theorem of this section, we need the following definition.

Definition 3.2. Let $G$ be a local function such that $E^{\mu_{r}}[G]=0$. For any vector $m=\left\{m_{\alpha}\right\}_{\alpha=0}^{d}$ we define the "variance" $V_{\ell}(G, m)$ by

$$
\begin{aligned}
V_{\ell}(G, m)= & \left(2 \ell_{1}+1\right)^{-d}\left\langle\left[\sum_{|x| \leqq \ell_{1}}\left(\tau_{x} G-\alpha_{\ell}(G)\right)\right]\left(-\mathscr{L}_{s, \ell}\right)^{-1}\right. \\
& \left.\times\left[\sum_{|x| \leqq \ell_{1}}\left(\tau_{x} G-\alpha_{\ell}(G)\right)\right]\right\rangle_{\mu_{\ell, m}},
\end{aligned}
$$


where $\ell_{1}=\ell-\ell^{1 / d^{2}}$ and $\mu_{\ell, m}$ is the canonical Gibbs state of $(2 \ell+1)^{d}$ sites with parameters such that the mean of $I_{\alpha}, \alpha \geqq 0$, on the block $\Lambda_{\ell}$ are equal to $m_{\alpha}$, namely,

$$
\mu_{\ell, m}=\Xi_{\ell, m}^{-1} \prod_{\alpha=0}^{d} \delta\left(\bar{I}_{\alpha, \ell}-m_{\alpha}\right) \mu_{\ell} .
$$

Here $\mu_{\ell}$ denotes the counting measure on the configurations in $\Lambda_{\ell}, \Xi_{\ell, m}$ is the normalization and

$$
\alpha_{\ell}(G)=E^{\mu_{r}}\left[G \mid \bar{I}_{\ell}^{+}\right],
$$

where $E^{\mu_{r}}\left[G \mid \bar{I}_{\ell}^{+}\right]$is the conditional expectation given the averages $\bar{I}_{\ell}^{+1}$ :

$$
\bar{I}_{\alpha, \ell}=\underset{|y| \leqq \ell}{\operatorname{Av}_{\alpha}} I_{\alpha}(y):=\frac{1}{(2 \ell+1)^{d}} \sum_{|y| \leqq \ell} I_{\alpha}\left(\eta_{y}\right) \quad \alpha=0, \ldots, d .
$$

The generator $\mathscr{L}_{s, \ell}$ is the symmetric part of the generator restricted to $\Lambda_{\ell}$, in the sense that the sums in (2.2) and (2.4) are restricted to the sites and bonds contained in $\Lambda_{\ell}$. We define also the "variance" of $G$ by

$$
V(G, r)=\limsup _{\ell \rightarrow \infty} E^{\mu_{r}}\left[V_{\ell}\left(G, \bar{I}_{\ell}\right)\right] .
$$

In order to make the above definition meaningful, one needs to give sense to $\mathscr{L}_{s, \ell}^{-1}$. This is a consequence of the finite volume ergodicity of the generator, summarized in the next Theorem 3.3. We will use the following notation: given a configuration $\eta$ on a box $\Lambda_{\ell}$ of size $\ell$, we put

$$
N_{\alpha}(\eta)=\sum_{x \in \Lambda_{\ell}} I_{\alpha}\left(\eta_{x}\right), \quad \alpha=0, \ldots, d
$$

Moreover, for any choice of $m_{\alpha}$, we denote by $\Omega_{\ell, m}$ the set of configurations $\eta$ in $\Lambda_{\ell}$ such that $N_{\alpha}(\eta)=m_{\alpha}(2 \ell+1)^{d}$ for $\alpha=0, \ldots, d$. Note that there are many choices of the $m_{\alpha}$ 's such that $\Omega_{\ell, m}$ is empty. We consider only the non-trivial choices.

We have the following

Theorem 3.3. The process generated by $\mathscr{L}_{s, \ell}$ on $\Omega_{\ell, m}$ endowed with the measure $\mu_{\ell, m}$ is ergodic.

Proof. Since the process is a finite Markov chain, it is sufficient to show that it is transitive, in the sense that, given any couple of configurations $\eta$ and $\bar{\eta}$ in $\Omega_{\ell, m}$, it is possible to find a sequence of jumps and collisions which transform $\eta$ into $\bar{\eta}$.

We first consider Model I.

Let

$$
N_{\alpha}^{-}(\eta)=\sum_{\xi \in \Lambda_{\ell}}\left[\eta\left(x, e_{\alpha}\right)+\eta\left(x,-e_{\alpha}\right)\right], \quad \alpha=1, \ldots, d, \quad \sum_{\alpha=1}^{d} N_{\alpha}^{-}(\eta)=N_{0}(\eta) .
$$

Step 1. If $\eta, \bar{\eta} \in \Omega_{\ell, m}$, then $N_{\alpha}^{-}(\eta)-N_{\alpha}^{-}(\bar{\eta})$ is even.

Proof of Step 1. If $N_{\alpha}^{-}(\eta)-N_{\alpha}^{-}(\bar{\eta})$ were odd, then there is at least $x \in \Lambda_{\ell}$ such that $\eta\left(x, e_{\alpha}\right)-\bar{\eta}\left(x, e_{\alpha}\right)= \pm 1$ and $\eta\left(x,-e_{\alpha}\right)-\bar{\eta}\left(x,-e_{\alpha}\right)=0$ or viceversa. Hence $\left[\eta\left(x, e_{\alpha}\right)-\eta\left(x,-e_{\alpha}\right)\right]-\left[\bar{\eta}\left(x, e_{\alpha}\right)-\bar{\eta}\left(x,-e_{\alpha}\right)\right]= \pm 1$. But $N_{\alpha}(\eta)=N_{\alpha}(\bar{\eta})$, so for each

\footnotetext{
${ }^{1}$ In Sect. 4 we will introduce other quantities $I_{\alpha}$ with negative indices. We use here the + exponent to emphasize that $\alpha$ here is non-negative.
} 
such $x$ there is an $y \in \Lambda_{\ell}$ such that $\left[\eta\left(y, e_{\alpha}\right)-\eta\left(y,-e_{\alpha}\right)\right]-\left[\bar{\eta}\left(y, e_{\alpha}\right)-\bar{\eta}\left(y,-e_{\alpha}\right)\right]=\mp 1$, to compensate the discrepancy of momenta in $x$. But this means that $N_{\alpha}^{-}(\eta)-N_{\alpha}^{-}(\bar{\eta})$ is not odd.

Step 2. Let $N_{\alpha}^{-}(\eta)-N_{\alpha}^{-}(\bar{\eta})=2 k_{\alpha}$. If $k_{\alpha}>0$ for some $\alpha=1, \ldots, d$, then there is a sequence of jumps and collisions which transform $\eta$ into $\bar{\eta}$.

Proof of Step 2. To simplify the argument, let $d=3$. We have $N_{0}(\eta)=N_{0}(\bar{\eta})$, hence $\sum_{\alpha=1}^{3} k_{\alpha}=0$. Without loss of generality we can assume $k_{1}<0$ and $k_{2} \geqq 0$, $k_{3} \geqq 0$. Therefore, with a suitable sequence of jumps, we can transform $\eta$ into a configuration $\eta_{1}$ with $N_{\alpha}\left(\eta_{1}\right)=N_{\alpha}(\eta)$ and $N_{\alpha}^{-}\left(\eta_{1}\right)=N_{\alpha}^{-}(\eta)$ such that there are at least $-k_{1}$ sites $x_{1}, \ldots, x_{-k_{1}}$, where there is no particle both with velocity $e_{1}$ and with velocity $-e_{1}$. In the same way, one can construct a configuration $\eta_{2}$ such that $N_{\alpha}\left(\eta_{2}\right)=N_{\alpha}(\bar{\eta})$ and $N_{\alpha}^{-}\left(\eta_{2}\right)=N_{\alpha}^{-}(\bar{\eta})$ such that there are $k_{2}$ sites $y_{1}, \ldots, y_{k_{2}}$ without particles with velocity $e_{2}$ and $-e_{2}$ and $k_{3}$ sites $y_{k_{2}+1}, \ldots, y_{k_{2}+k_{3}}$ without particles with velocities $e_{3}$ and $-e_{3}$. Since $k_{2}+k_{3}=-k_{1}$, there is no loss of generality assuming that the two sets of points coincide, because otherwise extra jumps can be done to reduce to this situation. Then we are in position to make a collision in each of the sites $x_{i}$, transforming $\eta_{1}$ into $\eta_{2}$.

Step 3. If $k_{\alpha}=0$ for each $\alpha=1, \ldots, 3$ then a sequence of jumps is sufficient to transform $\eta$ into $\bar{\eta}$.

Proof of Step 3. In fact, if $k_{\alpha}=0, \sum_{x \in \Lambda_{\ell}} \eta(x, v)=\sum_{x \in \Lambda_{\ell}} \bar{\eta}(x, v)$ for each $v \in \mathscr{V}$. Therefore jumps are enough to transform $\eta$ into $\bar{\eta}$.

As for Model II, we sketch the proof. Define $\eta \sim \zeta$ iff there exist a collision $q \in \mathscr{Q}$ and a site $y \in \Lambda_{L}$ such that $Q_{y}^{q} \eta=\zeta$. This defines an equivalence relation and we say $\eta$ and $\zeta$ are connected if they are in the same equivalence class. If $\eta$ is a configuration in 0 , we will also denote by $\eta$ the subset of $\mathscr{V}$ such that $\eta(v)=1$.

Lemma 3.4. For any two configurations $\eta$ and $\zeta$ at $0, \eta$ and $\zeta$ are connected iff $I^{+}(\eta)=I^{+}(\zeta)$.

Proof.

Step 1. We claim first the same result holds on the space $\mathscr{W}=\{( \pm 1, \pm 1)\}$. This can be checked easily.

Step 2. Next, we claim Lemma 3.4 holds if we restrict ourselves to the subspace $\mathscr{V}_{2}^{(3)}=\{( \pm 1, \pm 1, \pm \varpi)\}$. This can be checked easily too. We give a brief sketch. From the previous claim, the projection of $\eta$ and $\zeta$ onto the space $\mathscr{W}$ are connected. Let $\eta_{3}^{ \pm}=$the number of $\left\{v \in \eta\right.$ with $\left.v_{3}= \pm \varpi\right\}$. Since the momenta of $\eta$ and $\zeta$ are the same, $\eta_{3}^{ \pm}=\zeta_{3}^{ \pm}$. Hence we only have to prove the following:

Suppose $\{u, v\} \subset \eta$ with $u \neq v$. Let $\zeta=(\eta \backslash\{u, v\}) \cup\left\{\left(u_{1}, u_{2}, v_{3}\right),\left(v_{1}, v_{2}, u_{3}\right)\right\}$. Then $\zeta$ and $\eta$ are connected.

But $u+v=\left(u_{1}, u_{2}, v_{3}\right)+\left(v_{1}, v_{2}, u_{3}\right)$, hence we have proved the claim. This proposition is obvious and will be used repeatedly in the following proof. It basically means that the third component can be changed freely in $\mathscr{V}_{2}^{(3)}$.

Step 3. Suppose $\eta$ and $\zeta$ are two configurations in $\mathscr{V}_{2}^{(3)}$ with the same momentum but not the same number of particles. Suppose $I_{0}(\eta)>I_{0}(\zeta)$. We claim that $\eta$ is connected to $\zeta \cup A$ for some $A$ satisfying that $A \cap \zeta=\phi$ and $A$ is reflexive, i.e., if 
$v \in A$ then $-v \in A$. We first claim that $I_{0}(\eta)-I_{0}(\zeta)=2 n$ for some $n \in \mathscr{N}$ since otherwise $I_{3}(\eta) \neq I_{3}(\zeta)$. We now follow previous arguments by first considering the projection onto the space $\mathscr{W}$ and then adjusting the third components. In more details, we first prove our claim in the two dimensional space $\mathscr{W}$. This can be checked directly. We can then use the remark from Step 2 to adjust the third components. This proves the claim.

Step 4. Finally, the general case. Let $\eta^{(i)}=\left\{v \in \eta: v_{i}= \pm \varpi\right\}$. Clearly, $I_{\alpha}\left(\eta^{(i)}\right)=$ $I_{\alpha}\left(\zeta^{(i)}\right)$ for $\alpha>0$. Hence $\eta^{(i)}$ and $\zeta^{(i)}$ differ only for pairs of opposite vectors. Since any pair of opposite vectors can be brought into any different pair of opposite vectors by a collision, it is not hard to check $\eta$ and $\zeta$ are connected.

We now consider the general cases:

Lemma 3.5. For any two configurations $\eta^{\prime}$ and $\zeta$ on $\Lambda_{\ell}, \eta$ and $\zeta$ are connected iff $I^{+}(\eta)=I^{+}(\zeta)$.

Proof. The proof follows almost exactly the same arguments as in the previous lemma except some explanations are needed for the use of the simple exclusion. Let us illustrate it by proving Step 1 carefully. We are now in the setting of two dimensional configurations $\eta$ and $\zeta$ with the same momentum and number of particles. We now bring particles with opposite velocities to the same sites as much as possible. We can then decompose $\eta$ as the union of two configurations $\eta^{\text {pairs }}$, consisting of pairs of opposite vectors at the same sites, and the rest, $\eta^{\text {singles }}$. Since we have only four directions and no pairs are allowed, we can assume, without loss of generality, with obvious changes to cover the different situations, that $\eta^{\text {singles }}$ contains $\alpha$ particles in the direction $(1,1)$ and $\beta$ particles in the direction $(1,-1)$ and nothing else. Similarly, we can decompose $\zeta$ in the same way. Since $(1,1)$ and $(1,-1)$ are linearly independent, we can check that $\zeta^{\text {singles }}$ contains exactly the same number of particles in the directions $(1,1)$ and $(1,-1)$ and nothing else. Hence $\eta^{\text {pairs }}$ and $\zeta^{\text {pairs }}$ contain the same number of pairs because the total number of particles of $\eta$ and $\zeta$ are the same. We can connect $\eta^{\text {pairs }}$ and $\zeta^{\text {pairs }}$ by simple exclusions and collisions and we can also connect $\eta^{\text {singles }}$ and $\zeta^{\text {singles }}$ by simple exclusions. This concludes the proof of Step 1.

Step 2 is obvious. We now prove the claim in Step 3. First we check if instead of having the same number of particles and momentum in Step $1, \eta$ and $\zeta$ only have the same momentum (note they are now configurations on $\mathscr{W}$ ). From previous arguments we still have that $\eta^{\text {singles }}$ and $\zeta^{\text {singles }}$ have the same number of particles. Hence $\eta$ and $\zeta$ can be brought into configurations $\eta^{\prime}$ and $\zeta^{\prime}$ such that $\eta^{\prime} \backslash \zeta^{\prime}$ consists of pairs (assuming $I_{0}(\eta) \geqq I_{0}(\zeta)$ ). With this comment, we can prove Step 3 easily. Finally, Step 4 is by now straightforward.

The main theorem of this section is

Theorem 3.6. Let $\tilde{\Psi}_{t}$ be defined as above, $k=\ell \varepsilon^{-2 / d}$ and $f_{t}$ as in (2.31). Then, for $d \geqq 3$, and for any $\gamma>0$,

$$
\begin{aligned}
\lim _{l \rightarrow \infty} \lim _{\varepsilon \rightarrow 0} \varepsilon^{-2} s\left(f_{T} \mid \tilde{\Psi}_{T}\right) \leqq & C \lim _{l \rightarrow \infty} \lim _{\varepsilon \rightarrow 0} \int_{0}^{T} \varepsilon^{-2} s\left(f_{t} \mid \tilde{\Psi}_{t}\right) d t \\
& +\frac{C T}{\gamma} \sum_{\alpha, \beta=1}^{d} V\left(H_{\alpha}^{\beta}, r\right)+\text { const. } \gamma
\end{aligned}
$$


Here $C$ is a positive constant, $V$ is defined in (3.8) and

$$
H_{\alpha}^{\beta} \equiv H_{0, \alpha}^{\beta}, \quad H_{x, \alpha}^{\beta}=w_{x, \alpha}^{(a) \beta}-\sum_{\gamma, v=1}^{d} \tilde{D}_{\alpha \gamma}^{\beta, v} \nabla_{\gamma} I_{v}\left(\eta_{x}\right)-\mathscr{L}^{*} \tau_{x} F_{\alpha}^{\beta}
$$

where

$$
\tilde{D}_{\alpha \gamma}^{\beta}=D_{\alpha \gamma}^{\alpha}-\chi \delta_{\alpha, \gamma}
$$

We need the following lemmas. The first one is a large deviation bound; the other two are bounds on the entropy and the Dirichlet form.

Lemma 3.7. Let $\psi$ be the density given by (2.46) and let, for $\alpha=0, \ldots, d$,

$$
\begin{gathered}
\bar{m}_{x, k}^{\alpha}=E^{\psi}\left[\bar{I}_{\alpha, k}(x)\right] ; \quad \bar{I}_{\alpha, k}(x)=(2 k+1)^{-d} \sum_{|x-y| \leqq k} I_{\alpha}\left(\eta_{x}\right), \\
\zeta_{x, k}^{\alpha}=E^{\psi}\left[\bar{I}_{\alpha, k}(x) ; \bar{I}_{\alpha, k}(x)\right] \equiv E^{\psi}\left[\left(\bar{I}_{\alpha, k}(x)-\bar{m}_{x, k}^{\alpha}\right)^{2}\right],
\end{gathered}
$$

where $E[A ; B]=E[A B]-E[A] E[B]$. Then, for $k=\ell \varepsilon^{-2 / d}$ and for any $q<q_{0}$ with $q_{0}$ a small fixed constant,

$$
\lim _{\ell \rightarrow \infty} \lim _{\varepsilon \rightarrow 0} \varepsilon^{d-2} \log E^{\psi}\left[\exp \left\{q \sum_{x}\left[\left(\bar{I}_{\alpha, k}(x)-\bar{m}_{x, k}^{\alpha}(v)\right)^{2}-\zeta_{x, k}^{\alpha}\right]\right\}\right]=0 .
$$

In general, if $G_{x}=G_{x}\left(y_{0}, \ldots, y_{d}\right)$ is a family of bounded smooth functions such that

$$
G_{x}\left(\bar{m}_{x, k}\right)=\left.\frac{\partial G_{x}}{\partial y_{\alpha}}\right|_{y=\bar{m}_{x, k}}=0, \quad \alpha=0, \ldots, d,
$$

then for any smooth function $J$, there is $q_{0}$ such that for any $q<q_{0}$,

$$
\lim _{\ell \rightarrow \infty} \lim _{\varepsilon \rightarrow 0} \varepsilon^{d-2} \log E^{\psi}\left[\exp \left\{q \sum_{x} J(\varepsilon x)\left\{G_{x}\left(\bar{I}_{\alpha, k}(x)\right)-E^{\psi}\left[G_{x}\left(\bar{I}_{\alpha, k}(x)\right)\right]\right\}\right\}\right]=0 \text {. }
$$

This proof of Lemma 3.7 is similar to the one for Lemma 3.1 in [8], p. 1241. We omit the details.

Lemma 3.8. The density $f_{t}$ satisfies the following bounds:

(i)

$$
\frac{d}{d t} s\left(f_{t}\right) \leqq- \text { const. } \varepsilon^{-2+d} D_{\Lambda_{L}}\left(\sqrt{f_{t}}\right),
$$

where $D_{\Lambda_{L}}(g)=\left(D_{\Lambda_{L}}^{e x}+D_{\Lambda_{L}}^{c}\right)(g)$ is the Dirichlet form. Here

$$
D_{\Lambda_{L}}^{e x}(g)=\sum_{x \in \Lambda_{L}} \sum_{v \in \mathscr{V}} \sum_{\alpha=1}^{d} \chi \int\left[\nabla_{x, \alpha}^{v} g(\eta)\right]^{2} d \mu_{r}(\eta)
$$

with

$$
\nabla_{x, \alpha}^{v} g(\eta)=g\left(\eta^{x, x+e_{\alpha}, v}\right)-g(\eta)
$$

and $D_{\Lambda_{L}}^{c}(g)$ is given by

$$
D_{\Lambda_{L}}^{c}(g)=\sum_{x \in \Lambda_{L}} \sum_{q \in \mathscr{Q}} \int\left[\left(Q_{x}^{q} g\right)(\eta)\right]^{2} d \mu_{r}(\eta) .
$$


(ii) For any $t \geqq 0, \varepsilon^{-2} s\left(f_{t}\right) \leqq$ const.

(iii) For any local function $F$ and any $J$,

$$
\left|\left[E^{f_{t}}-E^{\mu_{r}}\right]\left[\varepsilon^{d-1} \sum_{x} J(\varepsilon x) \tau_{x} F\right]\right| \leqq \text { const . }
$$

Lemma 3.9. The relative entropy $s\left(f_{t} \mid \tilde{\Psi}_{t}\right)$ satisfies the bound

(i)

$$
\frac{d}{d t} s\left(f_{t} \mid \tilde{\Psi}_{t}\right) \leqq \varepsilon^{d} \int f_{t} \tilde{\Psi}_{t}^{-1}\left(\varepsilon^{-2} \mathscr{L}^{*}-\frac{\partial}{\partial t}\right) \tilde{\Psi}_{t} d \mu_{r}
$$

(ii) There is a constant $c_{t}$ independent of $f_{t}$ such that

$$
\lim _{\varepsilon \rightarrow 0} \varepsilon^{-2}\left[\frac{d}{d t} s\left(f_{t} \mid \tilde{\Psi}_{t}\right)-\varepsilon^{d} \int f_{t}\left(\varepsilon^{-2} \mathscr{L}^{*}-\frac{\partial}{\partial t}\right) \log \tilde{\Psi}_{t} d \mu_{r}\right]-c_{t} \leqq 0
$$

(iii)

$$
\int \mathscr{L}^{*} \tilde{\Psi}_{t} d \mu_{\beta}=0=\int \frac{\partial}{\partial t} \tilde{\Psi}_{t} d \mu_{r}
$$

Lemma 3.8 is obtained by adapting arguments from [24]. The first equation of Lemma 3.9 is proven in $[2,10]$. The last equation is obvious. The second equation differs from the first one only because the term $\tilde{\Psi}_{t}^{-1} \mathscr{L}^{*} \tilde{\Psi}_{t}$ is replaced by $\mathscr{L}^{*} \log \tilde{\Psi}_{t}$. From Lemma 2.2, their difference is small. Details were given in [8], Lemma 4.4, p. 1245 .

From now on we use the notation "const." for any constant independent of $f_{t}$ and of the configuration (but may depend on the initial state and the parameters $\lambda$ ). We shall not compute these constants at each step but will use Eq. (iii) of Lemma 3.9 to determine the final constant.

Proof of Theorem 3.6. By Lemma 3.9 to estimate the derivative of the relative entropy it is enough to compute the quantity

$$
\mathscr{I} \equiv \varepsilon^{d-2} E^{f_{t}}\left[\left(\varepsilon^{-2} \mathscr{L}^{*}-\frac{\partial}{\partial t}\right) \log \tilde{\Psi}_{t} d \mu_{r}\right] .
$$

We first compute $\varepsilon^{d-4} \mathscr{L}^{*} \log \tilde{\Psi}_{t}$ :

$$
\begin{aligned}
\varepsilon^{d-4} \mathscr{L}^{*} \log \tilde{\Psi}_{t}= & \varepsilon^{d-4}\left(\mathscr{L}^{e x *}\left(\log \tilde{\Psi}_{t}-\varepsilon^{2} \Phi\right)+\varepsilon^{d-2} \mathscr{L}^{*} \Phi\right. \\
= & \varepsilon^{d-3} \sum_{x} \sum_{\alpha, \beta=1}^{d}\left(\lambda_{\alpha} * \hat{\omega}\right)(\varepsilon x, t) \nabla_{\beta}^{-} w_{x, \beta}^{* \alpha} \\
& \quad+\varepsilon^{d-2} \sum_{x} \sum_{\alpha=1}^{d}\left(\lambda_{0} * \hat{\omega}\right)(\varepsilon x, t) \nabla_{\beta}^{-} w_{x \alpha}^{* 0}+\varepsilon^{d-2} \mathscr{L}^{*} \Phi \\
= & A_{1}+A_{2}+A_{3} .
\end{aligned}
$$

The term $A_{2}$ is easier than $A_{1}$ because of the factor $\varepsilon^{d-2}$. We first sum by parts. The difference operator is thus moved to act on $\lambda_{0} * \hat{\omega}$. Recall the expression of the current given by (3.3). We can separate the current into the symmetric part and the asymmetric part. Let $A_{2}=B_{1}+B_{2}$ with $B_{1}$ ( $B_{2}$ resp.) related to the 
symmetric (asymmetric resp.) part. Hence

$$
\begin{gathered}
B_{1}=\chi \varepsilon^{d-2} \sum_{x} \sum_{\beta=1}^{d} \nabla_{\beta}^{-} \nabla_{\beta} \lambda_{0}\left[\hat{\omega} * I_{0}\right]_{x}, \\
B_{2}=\varepsilon^{d-2} \sum_{x} \sum_{\alpha=1}^{d} \nabla_{\alpha}^{-} \lambda_{0}(\varepsilon x, t)\left(\hat{\omega} * w_{x \alpha}^{(a)}{ }^{0}\right),
\end{gathered}
$$

where $\left[\hat{\omega} * I_{\alpha}\right]_{x}=\sum_{y} \hat{\omega}(x-y) I_{\alpha}\left(\eta_{y}\right)$. From the Taylor expansion we can approximate $\nabla_{\beta}^{-} \nabla_{\beta}$ by $\varepsilon^{2} \partial_{z_{\beta}} \partial_{z_{\beta}}$ with a negligible lower order term.

Since our density $f_{t}$ is very close to the equilibrium, we can compute $B_{1}$ to great accuracy. Because $E^{\mu_{r}}\left[B_{1}\right]=0$, we have from (ii) and (iii) of Lemma 3.8,

$$
\left|E^{f}\left(B_{1}\right)\right|=\left|E^{f}\left(B_{1}\right)-E^{\mu,}\left[B_{1}\right]\right| \leqq \varepsilon .
$$

Hence $B_{1}$ is negligible.

We now examine the difficult term $A_{1}$.

$$
\begin{aligned}
A_{1} & =-\varepsilon^{d-3} \sum_{x} \sum_{\alpha, \beta=1}^{d}\left(\nabla_{\beta} \lambda_{\alpha}\right)(\varepsilon x, t)\left[\hat{\omega} * w_{y, \alpha}^{* \beta}\right]_{x} \\
& =-\chi \varepsilon^{d-3} \sum_{x} \sum_{\alpha, \beta=1}^{d} \nabla_{\beta}^{-} \nabla_{\beta} \lambda_{\alpha}\left(\hat{\omega} * I_{\alpha}\right)_{x}-\varepsilon^{d-3} \sum_{x} \sum_{\alpha, \beta=1}^{d} \nabla_{\beta} \lambda_{\alpha}\left[\hat{\omega} * w_{y, \alpha}^{(a) \beta}\right]_{x} .
\end{aligned}
$$

As remarked after (3.15), we can replace $\varepsilon^{-2} \nabla_{\alpha}^{-} \nabla_{\alpha}$ by $\partial_{z_{\alpha}} \partial_{z_{\alpha}}$ up to a negligible error term.

To deal with the currents appearing in $A_{1}$ and in $B_{2}$, we need to look at their expectations w.r.t. suitable grand canonical measures modeled according to empirical averages of the conserved quantities. To this end, for any $Y=\left(Y_{0}, \ldots, Y_{d}\right)$, let $\mu_{1, Y}$ be the grand canonical measure such that

$$
E^{\mu_{1, Y}}\left[I_{\alpha}\right]=Y_{\alpha}, \quad \alpha=0, \ldots, d .
$$

The measure $\mu_{1, Y}$ is uniquely defined because it is required to be an invariant measure of the dynamics (exclusion+collision). Explicitly, $\mu_{1, Y}$ is the product measure with marginal at one point given by

$$
\mu_{1, Y}(\eta)=Z_{r, n}^{-1} \exp \left\{\sum_{\alpha=0}^{d} n_{\alpha}^{(Y)} I_{\alpha}(\eta)\right\} \mu_{r}(\eta),
$$

for $\eta=\{\eta(v), v \in \mathscr{V}\}$. The chemical potentials $n_{\alpha}^{(Y)}$ are suitably chosen to give the correct averages (3.16). The subscript 1 is used because we will need the grand canonical measures for the symmetric simple exclusion which will be introduced later on and carries a subscript 2.

Given an integer $k>0$ for any configuration $\eta$ in $\Lambda_{L}$, we consider the local empirical averages $\bar{I}_{\alpha, k}(x), \alpha=0,1, \ldots, d$, defined in (3.9). The measure $\mu_{1, \bar{I}_{k}^{+}(x)}$ is defined according to (3.17), with $Y_{\alpha}=\bar{I}_{\alpha, k}(x) . n_{\alpha}^{(Y)}=n_{\alpha}^{\left(\bar{I}_{k}(x)\right)}, \alpha=0, \ldots, d$ the corresponding chemical potentials; here $\bar{I}_{k}^{+}(x)=\left\{\bar{I}_{\alpha, k}(x), \alpha=0, \ldots, d\right\}$. Moreover 
we put

$$
f\left(v, n^{(Y)}\right):=\frac{e^{n_{0}^{(Y)}+\underline{n}^{(Y)}} \cdot v}{1+e^{n_{0}^{(Y)}+\underline{n}^{(Y)}} \cdot v} .
$$

With above notations, we have, for $Y_{\alpha}:=\bar{I}_{\alpha,}(x)$

$$
\begin{aligned}
& \bar{I}_{0, k}(x)=E^{\mu_{1, I_{k}(x)}\left[I_{0}\right]}=\sum_{v \in \mathscr{V}} f\left(v, n^{(Y)}\right), \\
& \bar{I}_{\alpha, k}(x)=E^{\mu_{1, I_{k}(x)}\left[I_{\alpha}\right]}=\sum_{v \in \mathscr{V}}\left(v \cdot e_{\alpha}\right) f\left(v, n^{(Y)}\right) .
\end{aligned}
$$

We can now define

$$
\begin{aligned}
& g_{x, \beta}^{\alpha}=\left(\hat{\omega} * w_{y, \beta}^{(a) \alpha}\right)_{x}-E^{\mu_{1, I_{k}(x)}}\left[\left(\hat{\omega} * w_{y, \alpha}^{(a)}\right)_{x}\right] ; \\
& g_{x, \alpha}^{0}=\left(\hat{\omega} * w_{y, \alpha}^{(a)}{ }^{0}\right)_{x}-E^{\mu_{1, I_{k}(x)}}\left[\left(\hat{\omega} * w_{y, \alpha}^{(a)}{ }^{0}\right)_{x}\right], \quad \alpha=1, \ldots, d
\end{aligned}
$$

From previous definitions we have,

$$
\begin{aligned}
& E^{\mu_{1, Y}}\left[\left(\hat{\omega} * w_{y, \beta}^{(a) \alpha}\right)_{x}\right]=\sum_{v \in \mathscr{V}}\left(v \cdot e_{\alpha}\right)\left(v \cdot e_{\beta}\right)\left\{\left[f\left(v, n^{(Y)}\right)\right]^{2}-f\left(v, n^{(Y)}\right)\right\}:=-\pi^{\alpha \beta}(Y), \\
& E^{\mu_{1, Y}}\left[\left(\hat{\omega} * w_{y, \alpha}^{(a)}{ }^{0}\right)_{x}\right]=\sum_{v \in \mathscr{V}}\left(v \cdot e_{\alpha}\right)\left\{\left[f\left(v, n^{(Y)}\right)\right]^{2}-f\left(v, n^{(Y)}\right)\right\}:=-\pi_{\alpha}^{0}(Y) .
\end{aligned}
$$

Equations (3.21) are not explicit in terms of the conserved quantities. To obtain an explicit expression one has to solve (3.19). For our purpose, some approximation will be sufficient. This will be carried out later on in this section.

Summarizing, we have

$$
\begin{aligned}
A_{1}= & \chi \varepsilon^{d-1} \sum_{x} \sum_{\alpha, \beta=1}^{d} \partial_{z_{\alpha} z_{\alpha}}^{2} \lambda_{\beta}\left[\hat{\omega} * I_{\beta}\right]_{x}-\varepsilon^{d-3} \sum_{x} \sum_{\alpha, \beta=1}^{d}\left(\nabla_{\alpha} \lambda_{\beta}\right)(\varepsilon x, t) \pi^{\alpha \beta}\left(\bar{I}_{k}^{+}(x)\right) \\
& +\varepsilon^{d-3} \sum_{x} \sum_{\alpha, \beta=1}^{d}\left(\nabla_{\alpha} \lambda_{\beta}\right)(\varepsilon x, t) g_{x, \alpha}^{\beta}+o(1) .
\end{aligned}
$$

Let

$$
D_{\alpha, \gamma}^{\beta}=\tilde{D}_{\alpha, \gamma}^{\beta}+\delta_{\alpha, \gamma} \chi
$$

We can rewrite

$$
\begin{aligned}
A_{1}= & \varepsilon^{d-1} \sum_{x} \sum_{\alpha, \beta, \gamma, v=1}^{d} D_{\alpha, \gamma}^{\beta, \nu}\left(\partial_{z_{\alpha}} \partial_{z_{\gamma}} \lambda_{\beta}\right)(\varepsilon x, t)\left[\hat{\omega} * I_{v}\right]_{x} \\
& +\varepsilon^{d-3} \sum_{x} \sum_{\alpha, \beta=1}^{d}\left(\partial_{z_{\alpha}} \lambda_{\beta}\right)(\varepsilon x, t)\left[g_{x \alpha}^{\beta}-\sum_{\gamma, v=1}^{d} \tilde{D}_{\alpha, \gamma}^{\beta, v}\left(\hat{\omega} * \nabla_{\gamma} I_{\nu}\left(\eta_{y}\right)\right)_{x}\right] \\
& -\varepsilon^{d-3} \sum_{x} \sum_{\alpha, \beta=1}^{d}\left(\nabla_{\alpha} \lambda_{\beta}\right)(\varepsilon x, t) \pi^{\alpha \beta}\left(\bar{I}_{k}^{+}(x)\right)+o(1) .
\end{aligned}
$$


Similarly, $B_{2}=C_{3}+B_{3}$ where

$$
\begin{aligned}
& C_{3}=\varepsilon^{d-1} \sum_{x, \alpha} \partial_{z_{\alpha}} \lambda_{0} g_{x, \alpha}^{0}, \\
& B_{3}=\varepsilon^{d-2} \sum_{x, \alpha} \nabla_{\alpha} \lambda_{0} \pi_{\alpha}^{0}\left(\bar{I}_{k}^{+}(x)\right) .
\end{aligned}
$$

From (3.15), (3.23) we rearrange the remaining terms as

$$
A_{1}+A_{2}+A_{3}=C_{1}+C_{2}+C_{3}+B_{3}+o(1),
$$

where

$$
C_{1}=\varepsilon^{d-3} \sum_{x} \sum_{\alpha, \beta=1}^{d}\left(\partial_{z_{\alpha}} \lambda_{\beta}\right)(\varepsilon x, t)\left[g_{x \alpha}^{\beta}-\sum_{\gamma=1}^{d} \tilde{D}_{\alpha, \gamma}^{\beta}\left(\hat{\omega} * \nabla_{\gamma} I_{\beta}\left(\eta_{y}\right)\right)_{x}-\left(\hat{\omega} * \mathscr{L}^{*}\left(\tau_{y} F_{\alpha}^{\beta}\right)\right)_{x}\right],
$$

and

$$
C_{2}=\sum_{x} \sum_{\alpha, \beta=1}^{d}\left[\varepsilon^{d-1} \sum_{\gamma=1}^{d} D_{\alpha, \gamma}^{\beta}\left(\partial_{z_{\alpha}} \partial_{z_{\gamma}} \lambda_{\beta}\right)(\varepsilon x, t) \bar{I}_{\beta, k}(x)-\varepsilon^{d-3}\left(\nabla_{\alpha} \lambda_{\beta}\right)(\varepsilon x, t) \pi^{\alpha \beta}\left(\bar{I}_{k}^{+}(x)\right)\right] .
$$

We also need to compute

$$
\begin{aligned}
& -\varepsilon^{d-2} E^{f}\left\{\frac{\partial}{\partial t} \log \tilde{\Psi}_{t}\right\} \\
& =-E^{f}\left[\varepsilon^{d-1} \sum_{x} \sum_{\alpha=1}^{d} \frac{\partial}{\partial t} \lambda_{\alpha}(\varepsilon x \cdot t) \bar{I}_{\alpha, k}(x)+\varepsilon^{d} \sum_{x} \frac{\partial}{\partial t} \lambda_{0}(\varepsilon x, t) \bar{I}_{0, k}(x)\right]+\text { const } .
\end{aligned}
$$

Using an argument similar to the one explained in bounding $B_{1}$, one can prove that the second term on the right side is negligible up to a constant.

Summarizing these computations, we have

$$
\mathscr{I}=E^{f}\left[C_{1}+C_{3}+C_{4}\right]+\text { const. }+o(1),
$$

where

$$
C_{4}=\varepsilon^{d-2} \sum_{x} \Gamma_{x}\left(\bar{I}_{k}^{+}(x)\right)
$$

and

$$
\begin{aligned}
\Gamma_{x}(Y)= & \varepsilon \sum_{\alpha=1}^{d}\left\{\left(-\frac{\partial}{\partial t} \lambda_{\alpha}+\sum_{\beta, \gamma=1}^{d} D_{\beta \gamma}^{\alpha, v} \partial_{z_{\beta}} \partial_{z_{\gamma}} \lambda_{\alpha}\right)(\varepsilon x, t) Y_{v}\right. \\
& \left.-\varepsilon^{-2} \sum_{\beta=1}^{d} \nabla_{\alpha} \lambda_{\beta}(\varepsilon x, t) \pi^{\alpha \beta}(Y)-\varepsilon^{-1} \nabla_{\alpha} \lambda_{0}(\varepsilon x, t) \pi^{0}(Y)\right\} .
\end{aligned}
$$

The terms $E^{f}\left[C_{1}\right]$ and $E^{f}\left[C_{3}\right]$ are dealt with by using the following theorem. 
Theorem 3.10. Suppose $h$ is a local function, $J$ is a smooth function and $\psi$ is a local Gibbs state with smooth slowly varying chemical potentials $\lambda_{\alpha}(\varepsilon x)$ :

$$
\psi(\eta)=\frac{1}{Z} \exp \left[\varepsilon \sum_{x \in \Lambda_{L}} \sum_{\alpha=1}^{d} \lambda_{\alpha}(\varepsilon x) I_{\alpha}\left(\eta_{x}\right)\right]
$$

For any normalized positive $f$ and for any $\gamma>0$,

$$
\begin{aligned}
& \lim _{\ell \rightarrow \infty} \lim _{\varepsilon \rightarrow 0}\left\{\varepsilon^{d-2} \int f \sum_{x \in \Lambda_{L}} J(\varepsilon x)\left\{(\hat{\omega} * \tau h)(x)-E^{\mu_{1, I_{k}^{+}(x)}}[h]\right\} d \mu_{\beta}\right. \\
& \text {-const. } \left.\gamma^{-1} \int J(z)^{2} d z V(h, r)-\gamma \varepsilon^{d-4} D_{\Lambda_{L}}(\sqrt{f})-\delta^{-1} \varepsilon^{-2} s(f \mid \psi)\right\} \leqq 0,
\end{aligned}
$$

provided $\delta$ is small enough. Here $\bar{\ell}=\ell^{d+2}, k=\ell \varepsilon^{-2 / d}$ and $V$ is defined in (3.8).

Corollary 3.11. For any $\gamma>0$, any $f$ and any Gibbs state $\psi$ such that $\varepsilon^{-2} s(f \mid \psi)$ is bounded,

$$
\begin{aligned}
& \lim _{\ell \rightarrow \infty} \lim _{\varepsilon \rightarrow 0} \varepsilon^{d-2} \int \sum_{x \in \Lambda_{L}} J(\varepsilon x)\left\{(\hat{\omega} * \tau h)(x)-E^{\mu_{1, I_{k}^{+}(x)}}[h]\right\} f d \mu_{r} \\
& \quad \leqq \frac{1}{2 \gamma} \int J^{2}(z) d z V(h, r)+\gamma \lim _{\varepsilon \rightarrow 0} \varepsilon^{d-4} D_{\Lambda_{L}}(\sqrt{f})+\delta^{-1} \lim _{\ell \rightarrow \infty} \lim _{\varepsilon \rightarrow 0} \varepsilon^{-2} s(f \mid \psi) .
\end{aligned}
$$

In particular, if $f_{t}$ is the density in Theorem 2.1 then for any $\gamma>0$,

$$
\begin{gathered}
\lim _{\ell \rightarrow \infty} \lim _{\varepsilon \rightarrow 0} \int_{0}^{T} d t\left\{\varepsilon ^ { d - 2 } \int \sum _ { x \in \Lambda _ { L } } J ( \varepsilon x ) \left[(\hat{\omega} * \tau g)(x)-E^{\left.\left.\left.\mu_{1, I_{k}^{+}(x)}[g]\right)\right] f_{t} d \mu_{r}\right\}}\right.\right. \\
\leqq \gamma^{-1} C T V(g, r)+\delta^{-1} \lim _{\ell \rightarrow \infty} \lim _{\varepsilon \rightarrow 0} \int_{0}^{T} d t \varepsilon^{-2} s\left(f_{t} \mid \tilde{\Psi}_{t}\right)+\text { const. } \gamma,
\end{gathered}
$$

where $C$ is some constant depending on $J$ and $\gamma$.

The proofs of both the theorem and its corollary are postponed to the last section.

From Corollary 3.11 we can bound $E^{f}\left[C_{1}\right]$ by a variance term and the Dirichlet form of the density $f$. Because we are interested in a time integrated inequality in Theorem 3.6, the Dirichlet form will be integrated in time and we can use (3.30). Hence

$$
E^{f}\left[C_{1}\right] \leqq \text { const. } \gamma^{-1} \sum_{\alpha, \beta=1}^{d} V\left(H_{\alpha}^{\beta}, r\right)+\delta^{-1} \varepsilon^{-2} s\left(f_{t} \mid \tilde{\Psi}_{t}\right)+\text { const. } \gamma+\text { const. }+o(1),
$$

where the $o(1)$ denotes an error negligible after time integration.

Similarly, we can bound $C_{3}$. This time we have an extra $\varepsilon$ factor and the variance term vanishes in the limit $\varepsilon \rightarrow 0$.

Summarizing,

$$
\mathscr{I} \leqq \text { const. } \gamma^{-1} \sum_{\alpha, \beta=1}^{d} V\left(H_{\alpha}^{\beta}, r\right)+\delta^{-1} \varepsilon^{-2} s\left(f_{t} \mid \tilde{\Psi}_{t}\right)+\text { const. } \gamma+E^{f}\left[C_{4}\right]+\text { const. }+o(1)
$$


The constant is determined by the condition

$$
E^{\tilde{\Psi}_{t}}\left[\varepsilon^{d-2} \sum_{x} \Gamma_{x}\left(\bar{I}_{k}^{+}(x)\right)+\text { const. }\right]=o(1)
$$

By Lemma 2.2 one can replace $\tilde{\Psi}_{t}$ by $\Psi_{t}$. Let $\bar{m}_{x, k}^{\alpha}=E^{\Psi_{t}}\left[\bar{I}_{\alpha, k}(x)\right], \alpha=0, \ldots, d$. From the central limit theorem for the product measure $\Psi_{t}, \bar{m}_{x, k}^{\alpha}=m_{x}^{\alpha}+O\left(k^{-d}\right)$, with $m_{x}^{\alpha}=E^{\Psi_{t}}[I(x)]$. Hence the constant in (3.31) equals to

$$
E^{\tilde{\Psi}_{t}}\left[\varepsilon^{d-2} \sum_{x} \Gamma_{x}\left(\bar{I}_{k}^{+}(x)\right)\right]+o(1)=\varepsilon^{d-2} \sum_{x} E^{\Psi_{t}}\left[\Gamma_{x}\left(m_{x}^{\alpha}\right)\right]+o(1) .
$$

Using the entropy inequality (2.50) with $\gamma=\varepsilon^{2-d}$ we get

$$
\begin{aligned}
\mathscr{I} \leqq & \varepsilon^{-2} s\left(f_{t} \mid \tilde{\Psi}_{t}\right)+\text { const. } \gamma^{-1} \sum_{\alpha, \beta=1}^{d} V\left(H_{\alpha}^{\beta}, r\right)+\delta^{-1} \varepsilon^{-2} s\left(f_{t} \mid \tilde{\Psi}_{t}\right)+\text { const. } \gamma \\
& +\varepsilon^{d-2} \log E^{\Psi_{t}}\left[\exp \left\{\sum_{x}\left[\Gamma_{x}\left(\bar{I}_{k}^{+}(x)\right)-\Gamma_{x}^{\alpha}\left(m_{x}^{\alpha}\right)\right]\right\}\right]+o(1) .
\end{aligned}
$$

We will use the large deviations Lemma 3.2 to bound last term in (3.32). To apply it, we have to check that the function $\Gamma_{x}$ satisfies the following condition:

$$
\left.\frac{\partial \Gamma_{x}}{\partial Y_{\alpha}}\right|_{Y_{\alpha}=m_{x}^{\alpha}}=o(\varepsilon), \quad \alpha=0, \ldots, d
$$

To check this we have to evaluate derivatives of $\pi^{\alpha \beta}(Y)$, defined in (3.21). To this end we compute $\pi^{\alpha \beta}(Y+\delta Y)-\pi^{\alpha \beta}(Y)$ to the first order in $\delta Y$. We use the notation $\delta Y=\left(\delta Y_{0}, \delta \underline{Y}\right)$. From (3.18) and (2.33) (and a simple computation), to the first order in $\delta Y$ we have

$$
\begin{aligned}
& f(v, n+\delta n)-f(v, n+\delta n)^{2}-\left(f(v, n)-f(v, n)^{2}\right) \\
& \quad=\left(\delta n_{0}+\delta \underline{n} \cdot v\right)[f(v, n)(1-f(v, n))(1-2 f(v, n))]
\end{aligned}
$$

with $\delta n=n^{(Y+\delta Y)}-n^{(Y)}$. Hence

$$
\begin{aligned}
\pi^{\alpha \beta} & (Y+\delta Y)-\pi^{\alpha \beta}(Y) \\
= & \sum_{v \in \mathscr{V}}\left(v \cdot e_{\alpha}\right)\left(v \cdot e_{\beta}\right)\left\{f(v, n+\delta n)-f(v, n+\delta n)^{2}-\left(f(v, n)-f(v, n)^{2}\right)\right\} \\
= & \sum_{v \in \mathscr{V}}\left(v \cdot e_{\alpha}\right)\left(v \cdot e_{\beta}\right)\left[\delta n_{0}+(\delta \underline{n} \cdot v)\right] f(v, n) \\
& \quad \times(1-f(v, n))(1-2 f(v, n))+O\left(\delta n^{2}\right) .
\end{aligned}
$$


Recall (2.34). Hence for $Y_{\alpha}=m_{\alpha}$ we have

$$
\begin{aligned}
\pi^{\alpha \beta} & (Y+\delta Y)-\pi^{\alpha \beta}(Y) \\
& =\sum_{v \in \mathscr{V}}\left[\delta n_{0}+(\delta \underline{n} \cdot v)\right]\left(v \cdot e_{\alpha}\right)\left(v \cdot e_{\beta}\right) f(v, n)(1-f(v, n))(1-2 f(v, n)) \\
& =\left[S+O\left(\varepsilon^{2}\right)\right] \delta n_{0} \delta_{\alpha, \beta}+\varepsilon T \sum_{v \in \mathscr{V}}\left(v \cdot e_{\alpha}\right)\left(v \cdot e_{\beta}\right)(\lambda \cdot v)(\delta \underline{n} \cdot v) \\
& =S \delta n_{0} \delta_{\alpha, \beta}+2 \varepsilon h(\theta)\left[B \lambda_{\alpha} \delta n_{\alpha} \delta_{\alpha, \beta}+C \frac{\lambda_{\alpha} \delta n_{\beta}+\lambda_{\beta} \delta n_{\alpha}}{2}\right]+O\left(\varepsilon^{2}\right),
\end{aligned}
$$

where $S$ and $T$ are constants depending on $\theta$ and can be computed explicitly. The analogous computation for $\pi_{\alpha}^{0}$ is much simpler because an extra factor $\varepsilon$ appears before $\pi_{\alpha}^{0}$. We have

$$
\begin{aligned}
\pi_{\alpha}^{0}(Y+\delta Y)-\pi_{\alpha}^{0}(Y)= & \sum_{v \in \mathscr{V}}\left[\delta n_{0}+(\delta \underline{n} \cdot v)\right]\left(v \cdot e_{\alpha}\right) f(v, n) \\
& \times(1-f(v, n))(1-2 f(v, n))+o(1) \\
= & t(\theta)(1-2 \theta) \delta n_{\alpha}+o(1) .
\end{aligned}
$$

Moreover,

$$
\begin{aligned}
& \delta Y_{\alpha}=t(\theta) \delta n_{\alpha}+o(1), \\
& \delta Y_{0}=t(\theta) \delta n_{0}+o(1) .
\end{aligned}
$$

Therefore, up to order $o(\varepsilon)$ we have

$$
\begin{aligned}
\left.\frac{\partial \Gamma_{x}}{\partial Y_{\alpha}}\right|_{Y_{\alpha}=m_{x}^{\alpha}}= & \varepsilon\left(-\frac{\partial}{\partial t} \lambda_{\alpha}(z, t)+\sum_{\beta, \gamma=1}^{d}\left(\partial_{z_{\beta}} \partial_{z_{\gamma}} \lambda_{\alpha}\right)(z, t) D_{\beta \gamma}^{\alpha}-\frac{1}{t(\theta)} \partial_{z_{\alpha}} p(z, t)\right. \\
& \left.-\frac{h(\theta)}{t(\theta)}\left[B \partial_{z_{\alpha}} \lambda_{\alpha}(z, t)^{2}+C \sum_{\beta=1}^{d} \lambda_{\beta}(z, t) \partial_{z_{\beta}} \lambda_{\alpha}(z, t)\right]\right)+o(\varepsilon),(3.37) \\
& \left.\frac{\partial \Gamma_{x}}{\partial Y_{0}}\right|_{Y_{\alpha}=m_{x}^{\alpha}}=\text { const. } \varepsilon \sum_{\alpha=1}^{d} \partial_{z_{\alpha}} \lambda_{\alpha}(z, t)+o(\varepsilon) .
\end{aligned}
$$

The leading terms in the right sides of (3.37) vanish when $\lambda_{\alpha}$ and $p$ are classical solutions of the incompressible Navier-Stokes equations.

Assuming this, the last term in (3.32) vanishes as $\ell \rightarrow \infty$. Applying Lemma 3.9 integrated on time we get

$$
\begin{aligned}
\underset{\ell \rightarrow \infty}{\limsup } \limsup _{\varepsilon \rightarrow 0} \varepsilon^{-2} s\left(f_{T} \mid \tilde{\Psi}_{T}\right) & \\
\leqq \liminf _{\ell \rightarrow \infty} \liminf _{\varepsilon \rightarrow 0} \int_{0}^{T}[ & \left(\text { const. }+\delta^{-1}\right) \varepsilon^{-2} s\left(f_{t} \mid \tilde{\Psi}_{t}\right) d t \\
& \left.+\frac{C T}{\gamma} \sum_{\alpha, \beta=1}^{d} V\left(H_{\alpha}^{\beta}, r\right)+\text { const. } \gamma\right] .
\end{aligned}
$$


The proof of Theorem 3.6 is then achieved by the use of the Gronwall lemma. We shall prove that there exists a positive diffusion matrix $D$ such that

$$
\inf _{F} \sum_{\alpha, \beta=1}^{d} V\left(H_{\alpha}^{\beta}, r\right)=0,
$$

with $H_{\alpha}^{\beta}$ defined in (3.11). Hence Theorem 2.1 follows from Theorem 3.6. Sections 4 and 5 are devoted to prove that (3.38) holds with $F_{\alpha}$ in a suitable class of local functions.

\section{The Structure Theorem}

In this section we define the spaces of functions we will work with, and characterize their elements. We use several results of [8 and 15] for the simple exclusion. We first introduce some notations. Since the reference density is fixed, we shall use the symbol $E^{\mu}$ instead of $E^{\mu_{r}}$.

With any $x \in \Lambda_{L}$ fixed consider the following alternative description of the configurations in $x$. Recall that $\#(\mathscr{V})=N$. We introduce $N^{2}$ numbers $c_{\alpha}(v)$ with $\alpha=-N+d+1, \ldots, 0,1, \ldots, d$ and $v \in \mathscr{V}$. We put

$$
I_{\alpha}\left(\eta_{x}\right)=\sum_{v \in \mathscr{V}} c_{\alpha}(v) \eta(x, v) .
$$

To be consistent with the previous definition of the conserved quantities $I_{\alpha}\left(\eta_{x}\right)$ for $\alpha=0, \ldots, d$, we put

$$
c_{0}(v)=a, \quad c_{\alpha}(v)=b e_{\alpha} \cdot v, \quad \alpha=1, \ldots, d
$$

with the constants $a$ and $b$ specified by the normalization conditions below. The constants $c_{\alpha}(v)$ for $\alpha<0$ are chosen so that the mapping $\eta_{x} \rightarrow\left\{I_{\alpha}\left(\eta_{x}\right)\right\}$ is invertible and the orthogonality conditions below are satisfied. Define

$$
\tilde{\eta}(\cdot, v)=\eta(\cdot, v)-\theta, \quad \theta:=\langle\eta(\cdot, v)\rangle=\frac{e^{r}}{1+e^{r}} .
$$

For any $f$ and $g$ functions of $\eta_{x}$ define the scalar product

$$
(f, g):=E^{\mu}\left[f\left(\eta_{x}\right) ; g\left(\eta_{x}\right)\right]=E^{\mu}\left[f\left(\eta_{x}\right) g\left(\eta_{x}\right)\right]-E^{\mu}\left[f\left(\eta_{x}\right)\right] E^{\mu}\left[g\left(\eta_{x}\right)\right] .
$$

Then we have the orthogonality relations:

$$
\left(\eta(\cdot, v), \eta\left(\cdot, v^{\prime}\right)\right)=\delta_{v, v^{\prime}} \theta(1-\theta) .
$$

We also require the conditions

$$
\left(I_{\alpha}\left(\eta_{x}\right), I_{\beta}\left(\eta_{x}\right)\right)=\delta_{\alpha, \beta}, \quad \alpha, \beta=-N+d+1, \ldots, 0,1, \ldots, d .
$$

For Model I for example we have $N=2 d$. Let

$$
\begin{aligned}
& I_{\alpha}\left(\eta_{x}\right)=[2 \theta(1-\theta)]^{-1 / 2} \sum_{v \in \mathscr{V}}\left(e_{\alpha} \cdot v\right) \eta(x, v), \quad \alpha=1, \ldots, d, \\
& I_{0}\left(\eta_{x}\right)=[2 d \theta(1-\theta)]^{-1 / 2} \sum_{v \in \mathscr{V}} \eta(x, v),
\end{aligned}
$$


and define $I_{\alpha}\left(\eta_{x}\right), \alpha=-d+1, \ldots,-1$ by

$$
I_{\alpha}:=\sum_{\beta=1}^{d} A_{\alpha \beta}\left[\eta\left(x, e_{\beta}\right)+\eta\left(x,-e_{\beta}\right)\right],
$$

where $A_{\alpha \beta}$ are chosen so that

$$
E^{\mu}\left[I_{\alpha}\left(\eta_{x}\right) ; I_{\beta}\left(\eta_{x}\right)\right]=\delta_{\alpha, \beta}, \quad \alpha, \beta=-d+1, \ldots, d .
$$

The relation (4.3) for $\alpha=0, \ldots, d$ holds because of the definition of $I_{\alpha}\left(\eta_{x}\right), \alpha=$ $0, \ldots, d$. It remains to choose $A_{\alpha \beta}$ so that (4.3) holds for $\alpha<0$. This can be done easily for $d \geqq 2$. In particular, for $d=3$ one can choose

$$
A_{-1 \beta}=[4 \theta(1-\theta)]^{-1 / 2}(1,-1,0) ; \quad A_{-2 \beta}=[12 \theta(1-\theta)]^{-1 / 2}(1,1,-2) .
$$

In general, we will use the notation $I^{-}, I^{+}$to mean the sets $\left\{I_{\alpha}, \alpha<0\right\},\left\{I_{\alpha}, \alpha \geqq 0\right\}$ and $\tilde{I}_{\alpha}$ will denote the centered variable

$$
\tilde{I}_{\alpha}=I_{\alpha}-E^{\mu}\left[I_{\alpha}\right]
$$

Definition 4.1. Let

$$
\begin{aligned}
\mathscr{G} & =\{g \text { local function of } \eta \text { satisfying }(4.5)\}, \\
E^{\mu}[g] & =0, \quad \sum_{x} E^{\mu}\left[g ; I_{\alpha}\left(\eta_{x}\right)\right]=0, \quad \alpha=0, \ldots, d .
\end{aligned}
$$

Let $v$ be the product measure such that $E^{v}\left[I_{\alpha}\right]=m_{\alpha}$ for $\alpha \geqq 0$ and $\hat{g}(m)=E^{v}[g]$. Then the second condition is equivalent to

$$
\left.\frac{\partial \hat{g}(m)}{\partial m_{\alpha}}\right|_{m=\bar{m}}=0, \quad \alpha=0, \ldots, d,
$$

where $m$ is the vector $\left\{m_{\alpha}, \alpha=0, \ldots, d\right\}$ and $\bar{m}_{\alpha}=E^{\mu_{r}}\left[I_{\alpha}\right], \alpha=0, \ldots, d$, are the values corresponding to the equilibrium measure $\mu_{r}$.

To make the index notation more transparent, we will reserve latin indices $i, j, \ldots$ to coordinate directions: $i, j=1, \ldots, d$; greek indices will be used as color indices.

Moreover, we will use the notation:

$$
\nabla_{j} g(\eta)=g\left(\tau_{e_{j}} \eta\right)-g(\eta)
$$

where $\left(\tau_{x} \eta\right)(y, v)=\eta(y+x, v)$ and $\left(\tau_{x} f\right)(\eta)=f\left(\tau_{x} \eta\right)$.

Let

$$
\mathscr{G}^{0}=\left\{\sum_{\alpha=0}^{d} \sum_{j=1}^{d} a_{\alpha, j} \nabla_{j} \tilde{I}_{\alpha}\left(\eta_{0}\right), a_{\alpha, j} \in \mathbb{R}\right\}
$$

be the space of the gradients of the conserved quantities. Define the semi-norm

$$
\|\cdot\|_{-1}^{2}=V(\cdot)
$$

with $V$ defined in Definition (3.2). It is not obvious that $\|g\|_{-1}<\infty$ for $g \in \mathscr{G}$. This is part of the next theorem, which is the main result of this section. We will say that two elements of $\mathscr{G}$ are equivalent if they differ by an element of $\mathscr{G}^{0}$ and will denote by $\mathscr{G} \backslash \mathscr{G}^{0}$ the quotient of $\mathscr{G}$ w.r.t. this equivalence relation. 
Theorem 4.2. For all $g \in \mathscr{G}$, one has $\|g\|_{-1}<\infty$. Furthermore, let $\bar{G}$ denote the closure of $\mathscr{G}$ under the seminorm $\|\cdot\|_{-1}$ and define

$$
\langle\langle g, h\rangle\rangle=\frac{1}{4}\left[\|g+h\|_{-1}+\|g-h\|_{-1}\right] .
$$

Then $\langle\langle\cdot, \cdot\rangle\rangle$ is an inner product and $\overline{\mathscr{G}}$ equipped with this inner product is a Hilbert space. Moreover $\overline{\mathscr{G}}$ can be decomposed as

$$
\overline{\mathscr{G}}=\overline{\mathscr{L}_{s}\left[\mathscr{G} \mathscr{G}^{0}\right] \oplus \mathscr{G} 0}:=\mathscr{H} .
$$

To prove this theorem, we first introduce the concept of degree. Denote by $B$ a finite set of couples $(x, v) \in \mathbb{Z}^{d} \times \mathscr{V}$ and $|B|$ is its cardinality. We put

$$
\eta_{B}=\prod_{(x, v) \in B}[\eta(x, v)-\theta] .
$$

For each positive integer $n$, we denote by $\mathscr{M}_{n}$ the space generated by all monomials of degree $n$ :

$$
\mathscr{M}_{n}=\left\{h \in \mathscr{G} ; h=\sum_{|B|=n} \alpha_{B} \eta_{B}\right\} .
$$

Notice that in this definition all but a finite number of coefficients $\alpha_{B}$ vanish because $h$ is a cylindric function. We shall refer to

$$
\mathscr{G}_{n}=\bigcup_{1 \leqq j \leqq n} \mathscr{M}_{j}
$$

as the space of cylindric functions of degree $n$. All cylindric functions $h$ can be decomposed as a finite combination of cylindric functions of finite degree: $\mathscr{G} \subset \bigcup_{n \geqq 1} \mathscr{M}_{n}$. Let $\pi_{n}$ be the projection onto $\mathscr{M}_{n}$ and $\mathscr{R}=\sum_{n \geqq 2} \pi_{n}$. Then, $\mathscr{R} \mathscr{G}$ is the space of functions of degree two or higher. We introduce on $\mathscr{R} \mathscr{G}$ the following bilinear form and the associated seminorm, based on the symmetric simple exclusion generator

$$
\langle\langle g, h\rangle\rangle_{e x}=\frac{1}{4}\left(V^{e x}(g+h)-V^{e x}(g-h)\right], \quad\|g\|_{-1, e x}^{2}=\langle\langle g, g\rangle\rangle_{\mathrm{ex}},
$$

with $V^{e x}(g)$ defined similarly to $V(g)$ in Definition 3.1 of the previous section, but with the generator $\mathscr{L}_{s}$ replaced by $\mathscr{L}_{s}^{e x}$. More precisely, let

$$
V_{\ell}^{e x}(g, m)=\left(2 \ell_{1}+1\right)^{-d}\left\langle\sum_{|x| \leqq \ell_{1}} \tau_{x} g\left(-\mathscr{L}_{s, \ell}^{e x}\right)^{-1} \sum_{|x| \leqq \ell_{1}} \tau_{x} g\right\rangle_{\mu_{\ell, m}}
$$

with $\ell_{1}=\ell-\ell^{1 / d^{2}}$. Here $\mu_{\ell, m}$ is the canonical Gibbs state of $(2 \ell+1)^{d}$ sites with $\bar{I}_{\alpha, \ell}=m_{\alpha}, \alpha=-N+d+1, \ldots, d$. Explicitly

$$
\mu_{\ell, m}=Z_{\ell, m}^{-1} \prod_{\alpha=-N+d+1}^{d} \delta\left(\bar{I}_{\alpha, \ell}-m_{\alpha}\right) \mu_{\ell} .
$$

Here $\mu_{\ell}$ denotes the counting measure on the configurations in $\Lambda_{\ell}$ and $Z_{\ell, m}$ is the normalization. With this notation,

$$
V^{e x}(g, r)=\limsup _{\ell \rightarrow \infty} E^{\mu_{r}}\left[V_{\ell}^{e x}\left(g, \bar{I}_{l}\right)\right] .
$$


We also introduce on $\mathscr{G}$ the inner product defined as

$$
\langle g, h\rangle_{r, 0}=\left\langle g, \sum_{x} \tau_{x} h\right\rangle_{\mu_{r}}=\left\langle\sum_{x} \tau_{x} g, h\right\rangle_{\mu_{r}} .
$$

We omit the label $r$ because it is fixed throughout this section.

Recall the following result from [8]. It is proved in [18] when the function $h$ is a current of a process. The general case is formulated and proved in [8] using a multiscale integration by parts lemma.

Theorem 4.3. The bilinear form $\langle\langle\cdot, \cdot\rangle\rangle_{\text {ex }}$ is a scalar product on $\mathscr{R} \mathscr{G}$. Moreover, we have the variational formula

$$
\langle\langle g, g\rangle\rangle_{e x}=\sup _{u \in \mathscr{R} \mathscr{G}}\left[\langle g, u\rangle_{0}-\frac{1}{4}\left\langle u,\left(-\mathscr{L}_{s}^{e x}\right) u\right\rangle_{0}\right] .
$$

Furthermore, the subspace $\mathscr{L}_{s}^{\text {ex }} \mathscr{R} \mathscr{G}$ is dense in $\mathscr{R} \mathscr{G}$ under the norm $\|\cdot\|_{-1 \text {,ex }}$ associated to the scalar product (4.11).

The subspace $\mathscr{R} \mathscr{G}$ is also denoted by $\mathscr{G}^{e x}$ because of the similarity with the space $\mathscr{G}$. Clearly, if we replace $\left(-\mathscr{L}_{s}^{e x}\right)$ on the right-hand side of $(4.10)$ by $\left(-\mathscr{L}_{s}\right)$ we have a lower bound. From the definition of $\|g\|_{-1}$, we have for all $g \in \mathscr{R} \mathscr{G}$,

$$
\|g\|_{-1} \leqq\|g\|_{-1, e x} .
$$

Let us summarize what we have so far. The space $\mathscr{G} \subset \bigcup_{n \geqq 1} \mathscr{M}_{n}$ can be decomposed into degrees, and the subspace of degree two or higher is

$$
\mathscr{R} \mathscr{G}=\bigcup_{n \geqq 2} \mathscr{M}_{n} .
$$

Let $\mathscr{G}_{-} \subset \mathscr{M}_{1}$ be the space of functions

$$
\mathscr{G}_{-}=\left\{\sum_{\alpha<0} a_{\alpha} \tilde{I}_{\alpha}\left(\eta_{0}\right), a_{\alpha} \in \mathbb{R}\right\} .
$$

From condition (4.5) the subspace of degree one is a subspace of $\mathscr{M}_{1}$ and is given by

$$
\mathscr{G} \cap \mathscr{M}_{1}=\mathscr{G}_{-} \oplus \mathscr{G}^{0} .
$$

Note that as a subspace of the space $\mathscr{G}$ with inner product $\langle\cdot, \cdot\rangle_{0}$, the space $\mathscr{G}^{0}$ is in the kernel of the inner product. We shall denote the completion of $\mathscr{G} / \mathscr{G}^{0}$ w.r.t. $\|\cdot\|_{-1}$ by $\mathscr{K}$. Note that we have not yet proved the norm $\|\cdot\|_{-1}$ is finite on $\mathscr{G} / \mathscr{G}^{0}$. This will be proved later on in Lemma 4.5 . By definition, $\mathscr{K}$ is generated by $\mathscr{R} \mathscr{G} \oplus \mathscr{G}_{-}$. To prove Theorem 4.2, the key estimate is contained in the following theorem:

Theorem 4.4. The bilinear form $\langle\langle\cdot, \cdot\rangle\rangle$ is a scalar product in $\mathscr{K}$ and we have the variational formula

$$
\langle\langle g, g\rangle\rangle=\sup _{u \in \mathscr{K}}\left[\langle g, u\rangle_{0}-\frac{1}{4}\left\langle u,\left(-\mathscr{L}_{s}\right) u\right\rangle_{0}\right] .
$$

Furthermore, the subspace $\mathscr{L}_{s} \mathscr{K}$ is dense in $\mathscr{K}$ w.r.t. the norm $\|\cdot\|_{-1}$. 
Assuming this result, we can conclude the proof of Theorem 4.2. Clearly, (4.8) follows from Theorem 4.4 and the decomposition of degree discussed in the previous paragraph before Theorem 4.4 . We next show that $\langle\langle\cdot, \cdot\rangle\rangle$ is an inner product. Let $v \in \mathscr{G}^{0}$. By definition,

$$
\begin{aligned}
\left\|-\mathscr{L}_{s} u+v\right\|_{-1}^{2}= & \limsup _{k \rightarrow \infty} V_{k}\left(-\mathscr{L}_{s} u\right)+\limsup _{k \rightarrow \infty}(2 k+1)^{-d} \\
& \times\left\langle\sum_{x:|x| \leqq k}-\tau_{x} \mathscr{L}_{s} u,-\mathscr{L}_{s, k}^{-1} \sum_{x:|x| \leqq k} \tau_{x} v\right\rangle+\limsup _{k \rightarrow \infty} V_{k}(v) .
\end{aligned}
$$

The first term converges to $\left\langle u,-\mathscr{L}_{s} u\right\rangle_{0}$. The second vanishes. The last term can be computed explicitly since $-\mathscr{L}_{s, k}^{-1} v$ has an explicit expression. This gives a formula for $\left\|-\mathscr{L}_{s} u+v\right\|_{-1}$ showing that the variational formula holds for $-\mathscr{L}_{s} u+v$. From Theorem 4.4 and the previous argument, elements of the form $-\mathscr{L}_{s} u+v$ are dense in $\mathscr{G}$. Hence the variational formula holds in general. This proves also that $\langle\langle\cdot, \cdot\rangle\rangle$ is an inner product and concludes Theorem 4.2.

Proof of Theorem 4.4. We first note that it suffices to prove that the subspace $\mathscr{L}_{s} \mathscr{K}$ is dense in $\mathscr{K}$ under the norm $\|\cdot\|_{-1}$. Assuming this, we only have to check (4.13) with $g$ replaced by $\mathscr{L}_{s} g$. But this follows from the definition $(4.7)$ of $\langle\langle\cdot, \cdot\rangle\rangle$ and Definition 3.1.

We now outline the proof that $\mathscr{L}_{s} \mathscr{K}$ is dense in $\mathscr{K}$. The detailed proofs of lemmas will be presented at the end of this section. We first state the following lemmas.

Step 1.

Lemma 4.5. Put $Q f \equiv \sum_{q \in \mathcal{Q}} Q_{x}^{q} f$. Then, given $\tilde{I}_{\alpha}\left(\eta_{x}\right)$, for $\alpha<0$, there exist a local function $h$ and a function $g \in \mathscr{R} \mathscr{G}$ such that

$$
\tilde{I}_{\alpha}\left(\eta_{x}\right)=\left(Q_{x} h\right)\left(\eta_{x}\right)+g\left(\eta_{x}\right) .
$$

Proof of Lemma 4.5. Fix $x=0$. For any $G \in \mathscr{G}$, we have $Q G \in \mathscr{R} \mathscr{G} \oplus \mathscr{G}_{-}$, because the null space of $Q$ is generated by $I_{\alpha}$ for $\alpha=0, \ldots, d$. Therefore, for any $z \in \mathscr{G}_{-}$ we can find a $g \in \mathscr{R} \mathscr{G}$ and $w \in \mathscr{G}_{-}$such that

$$
Q z=g+w .
$$

This relation defines a linear map from $\mathscr{G}_{-}$to itself. Our goal is to prove this map is surjective. Suppose it is degenerate. Then there is a $z$ such that $Q z=g$. Multiplying both sides by $z$ we have

$$
\langle z, Q z\rangle_{0}=0 \text {. }
$$

Clearly, $\mathscr{G}_{-}$has no nonvanishing intersection with the kernel of $Q$. This concludes the proof of Lemma 4.5.

Corollary 4.6. For any constant $a_{\alpha} \in \mathbb{R}$,

$$
\left\|\sum_{\alpha<0} a_{\alpha} \tilde{I}_{\alpha}\right\|_{-1}<\infty .
$$


Hence for any constant $c_{\alpha, j} \in \mathbb{R}$,

$$
\left\|\sum_{\alpha<0} \sum_{j=1}^{d} c_{\alpha, j} \nabla_{j} I_{\alpha}\right\|_{-1}=0 .
$$

Moreover, the seminorm $\|\cdot\|_{-1}$ is finite on $\mathscr{K}$.

Proof of Corollary 4.6. From the definition of the norm $\|\cdot\|_{-1}$, it is easy to prove that (4.16) follows from (4.15). We now prove (4.15). Clearly, $\|Q h\|_{-1}$ is bounded for any bounded local function $h$. In fact, $Q h=\mathscr{L}_{s} h-\mathscr{L}_{s}^{e x} h$. But $\left\|\mathscr{L}_{s} h\right\|_{-1}$ is finite from its definition. On the other hand, $\mathscr{L}_{s}^{e x} h \in \mathscr{G}^{\text {ex }}$ so that $\left\|\mathscr{L}_{s}^{\text {ex }} h\right\|_{-1} \leqq$ $\left\|\mathscr{L}_{s}^{e x} h\right\|_{-1, e x}<\infty$ by (4.12). Also, for $g \in \mathscr{R} \mathscr{G}$ one has $\|g\|_{-1} \leqq\|g\|_{-1, e x}<\infty$. Together with Lemma 4.5 , this proves the boundedness of $\left\|\tilde{I}_{\alpha}\left(\eta_{x}\right)\right\|_{-1}$ and thus concludes Corollary 4.6.

Step 2.

Lemma 4.7. For any local function $g \in \mathscr{R} \mathscr{G}$ and any $\varepsilon>0$ there is a local function $u \in \mathscr{R} \mathscr{G}$ such that

$$
\left\|\mathscr{R} \mathscr{L}_{s} u-g\right\|_{-1, e x} \leqq \varepsilon .
$$

The proof of Lemma 4.7, will be given later on. Now we conclude the argument for the proof of Theorem 4.4.

Step 3.

Lemma 4.8. For any $w \in \mathscr{G}_{-}$and any $\varepsilon>0$ there is a $g \in \mathscr{K}$ such that

$$
\left\|w-\mathscr{L}_{s} g\right\|_{-1} \leqq \varepsilon .
$$

Assuming this lemma, we now prove Theorem 4.4. Let $u \in \mathscr{R} \mathscr{G}$. Then from Lemma 4.7 there is $g \in \mathscr{R} \mathscr{G}$ such that

$$
\left\|u-\mathscr{R} \mathscr{L}_{s} g\right\|_{-1} \leqq \varepsilon .
$$

Let $w=(1-\mathscr{R}) \mathscr{L}_{s} g$. From Lemma 4.8 , there is an $h \in \mathscr{K}$ such that

$$
\left\|w-\mathscr{L}_{s} h\right\|_{-1} \leqq \varepsilon .
$$

We have thus proved that

$$
\left\|u-\mathscr{L}_{s}(g-h)\right\|_{-1} \leqq \varepsilon .
$$

This concludes the proof of Theorem 4.4.

Proof of Lemma 4.7. The proof follows from three steps. The first two steps establish a priori estimates on the solution, the third is a compactness argument that provides the conclusion. The a priori estimates hold for the full generator, not only for its symmetric part and they will be used in the most general form in next section, while in this section we only need the ones for the symmetric part. To shorten the formulas, we will use the notation

$$
S=\frac{1}{2}\left[\mathscr{L}^{e x}+\left(\mathscr{L}^{e x}\right)^{*}\right], \quad A=\frac{1}{2}\left[\mathscr{L}^{e x}-\left(\mathscr{L}^{e x}\right)^{*}\right], \quad Q=\mathscr{L}^{c}
$$


Moreover, we write

$$
Q=\sum_{x} Q_{x}
$$

Step 1. First a priori estimate.

Theorem 4.9. Let $g$ be as in Lemma 4.7. For any $\lambda>0$, let $u_{\lambda}$ be the solution of the following equation in $\mathscr{R} \mathscr{G}$ :

$$
(-\lambda+S+\mathscr{R} A+\mathscr{R} Q) u_{\lambda}=g .
$$

Then for any $\beta \geqq 0$ there is a constant $C_{g}(\beta)$ such that

$$
\sum_{n}\left(a+n^{\beta}\right) \sum_{x} D\left(u_{n}\right) \leqq C_{g}, \quad u_{n}=\pi_{n} u_{\lambda}
$$

for sufficiently large $a>0$. Recall that $D$ is the Dirichlet form associated to the generator $\mathscr{L}$.

Proof. Our goal is to estimate the collision term $Q$. The asymmetric part $A$ is dealt with in the same way as in [15]. We write

$$
u=\sum_{B} \alpha(B) \eta_{B}
$$

We can interpret $\alpha(B)^{2}$, up to some normalization, as the probability of finding "pseudo-particles" at the set $B$. We denote by $B_{v}$ the set of $x$ such that $(x, v) \in B$,

$$
\eta_{B_{v}}=\prod_{x \in B_{v}}(\eta(x, v)-\theta)
$$

so that

$$
\eta_{B}=\prod_{v \in V} \eta_{B_{v}}
$$

with $\theta$ the expected value of $\eta(0, v)$ w.r.t. the equilibrium measure. Moreover, for $U \subset \mathscr{V}$ we set

$$
\eta_{x}^{U}=\prod_{v \in U}(\eta(x, v)-\theta)
$$

Recall that the cardinality of $\mathscr{V}$ is $N$. Below, $U$ and $V$ will always denote subsets of $\mathscr{V}$. We can write

$$
u=\sum_{i=0}^{N} \sum_{|U|=i} \sum_{B \nsupseteq x} \alpha(B \cup U) \eta_{B} \eta_{x}^{U} .
$$

Given any positive integer $n$ we take the scalar product $\langle\cdot, \cdot\rangle_{0}$ of the (4.21) times $-u_{n}$. We get

$$
\lambda\left\langle u_{n}, u_{n}\right\rangle_{0}-\left\langle u_{n}, Q u\right\rangle_{0}-\left\langle u_{n}, S u\right\rangle_{0}-\left\langle u_{n}, A u\right\rangle_{0}=-\left\langle u_{n}, g\right\rangle_{0} .
$$

In this proof we will drop the index from the scalar product $\langle\cdot, \cdot\rangle_{0}$.

The right side of (4.24) vanishes unless $n \leqq N_{g}$, with $N_{g}$ the cardinality of the support of the local function $g$. Moreover, for any $\varepsilon>0$ we have,

$$
\left|\left\langle u_{n}, g\right\rangle\right| \leqq \varepsilon\left\langle u_{n},(-S) u_{n}\right\rangle+\varepsilon^{-1}\left\langle g,(-S)^{-1} g\right\rangle \leqq \varepsilon\left\langle u_{n},(-S) u_{n}\right\rangle+C(g, \varepsilon),
$$


and hence

$$
\sum_{n}\left(a+n^{\beta}\right)\left|\left\langle u_{n}, g\right\rangle\right| \leqq \varepsilon \sum_{n} n^{\beta} D^{e x}\left(u_{n}\right)+C(g, \varepsilon)
$$

because the sum is finite; later on $\varepsilon$ will be chosen as a small constant.

We now bound the left-hand side. The terms involving $-S$ give the Dirichlet form associated with symmetric simple exclusions, i.e.,

$$
\left\langle u_{n},-S u\right\rangle=D^{e x}\left(u_{n}\right),
$$

because $S$ preserves the degree of the polynomials and monomials of different degrees are orthogonal.

The terms involving $Q$ can be bounded as follows. We assume $n \geqq 2$, because $\mathscr{R} u_{1}=0$. Writing $Q=\sum_{x} Q_{x}$, for any fixed $x$ we have

$$
\begin{aligned}
\left\langle u_{n}, \mathscr{R} Q_{x} u\right\rangle & =\left\langle u_{n}, Q_{x} u\right\rangle \\
& =\sum_{i, j=1}^{N} \sum_{|U|=i,|V|=j} \sum_{B \nsupseteq x,|B|=n-i} \alpha(B \cup U) \alpha(B \cup V)\left\langle\eta_{B}^{2}\right\rangle\left\langle\eta_{x}^{U}, Q_{x} \eta_{x}^{V}\right\rangle .
\end{aligned}
$$

Hence

$$
\begin{aligned}
& \sum_{n}\left(a+n^{\beta}\right)\left\langle u_{n}, Q_{x} u\right\rangle \\
& =\sum_{n}\left(a+n^{\beta}\right) \sum_{i, j=1}^{N} \sum_{|U|=i,|V|=j B \nsupseteq x,|B|=n-i} \sum_{|U|=i,|V|=j} \alpha(B \cup U) \alpha(B \cup V)\left\langle\eta_{B}^{2}\right\rangle\left\langle\eta_{x}^{U}, Q_{x} \eta_{x}^{V}\right\rangle \\
& \quad=\sum_{n} \sum_{i, j=1}^{N}\left(a+(n+i)^{\beta}\right) \sum_{\mid=n} \alpha(B \cup U) \alpha(B \cup V)\left\langle\eta_{B}^{2}\right\rangle\left\langle\eta_{x}^{U}, Q_{x} \eta_{x}^{V}\right\rangle \\
& =-\sum_{n}\left(a+(n+1)^{\beta}\right) Q_{x, n}+\Omega_{x}^{\beta},
\end{aligned}
$$

where

$$
\begin{aligned}
Q_{x, n} & =\sum_{B \nsubseteq x,|B|=n}\left\langle\eta_{B}^{2}\right\rangle \sum_{i, j=1}^{N} \sum_{|U|=i,|V|=j}\left\langle\alpha(B \cup U) \eta_{x}^{U},\left(-Q_{x}\right) \alpha(B \cup V) \eta_{x}^{V}\right\rangle \\
& =\sum_{B \nsubseteq x,|B|=n}\left\langle\eta_{B}^{2}\right\rangle\left\langle\sum_{U \subset \mathscr{V}} \alpha(B \cup U) \eta_{x}^{U},\left(-Q_{x}\right) \sum_{V \subset \mathscr{V}} \alpha(B \cup V) \eta_{x}^{V}\right\rangle
\end{aligned}
$$

and

$$
\Omega_{x}=\sum_{n} \Omega_{x, n}
$$

with

$$
\begin{aligned}
\Omega_{x, n}:= & \sum_{B \nsubseteq x,|B|=n} \sum_{U \subset \mathscr{V}}\left[(n+|U|)^{\beta}-(n+1)^{\beta}\right] \alpha(B \cup U)\left\langle\eta_{B}^{2}\right\rangle \\
& \times\left\langle\eta_{x}^{U}, Q_{x} \sum_{V \subset \mathscr{V}} \alpha(B \cup V) \eta_{x}^{V}\right\rangle .
\end{aligned}
$$


Since $|U| \leqq N$, there is a constant $C$ depending on $N$ and $\beta$, such that

$$
(n+|U|)^{\beta}-(n+1)^{\beta} \leqq C(n+N)^{\beta-1}(|U|-1) .
$$

From the Schwartz inequality we have,

$$
\begin{aligned}
\left|\Omega_{x, n}\right| \leqq & C(n+N)^{\beta-1} \sum_{B 尹 x,|B|=n}\left\langle\eta_{B}^{2}\right\rangle \sum_{|U| \geqq 2}|\alpha(B \cup U)| \\
& \times\left\langle\sum_{V \subset \mathscr{V}} \alpha(B \cup V) \eta_{x}^{V},\left(-Q_{x}\right) \sum_{V \subset \mathscr{V}} \alpha(B \cup V) \eta_{x}^{V}\right\rangle^{1 / 2} \\
\leqq & C(n+N)^{\beta-1}\left[\sum_{|U| \geqq 2} \sum_{B \ni x,|B|=n} \alpha(B \cup U)^{2}\left\langle\eta_{B}^{2}\right\rangle\right]^{1 / 2} \\
& \times\left[\sum_{B \nsubseteq x,|B|=n}\left\langle\eta_{B}^{2}\right\rangle\left\langle\sum_{V \subset \mathscr{V}} \alpha(B \cup V) \eta_{x}^{V}, \quad\left(-Q_{x}\right) \sum_{V \subset \mathscr{V}} \alpha(B \cup V) \eta_{x}^{V}\right\rangle\right]^{1 / 2} .
\end{aligned}
$$

From the expression of $u(4.23)$ we have:

$$
\sum_{U \subset \mathscr{V},|U| \geqq 2} \sum_{B, x \notin B,|B|=n} \alpha(B \cup U)^{2}\left\langle\eta_{B}^{2}\right\rangle \leqq C \sum_{|m-n| \leqq N} E\left[u_{m}^{2} \mathbb{1}\left\{n_{x} \geqq 2\right\}\right],
$$

where $\mathbb{1}\left\{n_{x} \geqq 2\right\}$ is the characteristic function of the event that there are at least two particles at $x$. The definition of $Q_{x, n}$ and previous inequality show that

$$
\left|\Omega_{x, n}\right| \leqq C(n+N)^{\beta-1}\left\{\sum_{|m-n| \geqq N} E\left[u_{m}^{2} \mathbb{1}\left\{n_{x} \geqq 2\right\}\right]\right\}^{1 / 2} Q_{x, n}^{1 / 2} .
$$

The following lemma can be proved with a technique introduced in [15].

Lemma 4.10. Let $\omega$ be a bounded function satisfying for some fixed constant $\ell$ that $\omega\left(x_{1}, \ldots, x_{n}\right)=0$ unless $\left|x_{i}-x_{j}\right| \leqq \ell$ for some $i \neq j$. Then there is a constant $C>0$ independent of $n$ such that

$$
\sum_{x} E\left[u_{n}^{2} \omega\right]-C n D^{e x}\left(u_{n}\right) \leqq 0
$$

Given Lemma 4.10, we have

$$
\sum_{x}\left|\Omega_{x, n}\right| \leqq C(n+N)^{\beta-1} \sqrt{n} \sum_{x} Q_{x, n}+C(n+N)^{\beta-1} \sqrt{n} \sum_{|m-n| \leqq N} D^{e x}\left(u_{m}\right)
$$

Hence, for $n$ sufficiently large the error term can be bounded with a small fraction of the Dirichlet form; more precisely, for any $\delta>0$ we can choose $a>0$ sufficiently large, so that

$$
\sum_{x}\left|\Omega_{x, n}\right| \leqq \delta\left(a+n^{\beta}\right) D\left(u_{n}\right) .
$$

The term involving $A$ is bounded in a similar way following a procedure introduced in [15] where the following proposition is proved: 
Proposition 4.11. For any $u \in \mathscr{R} \mathscr{G}$, there is a constant $C$ independent of $n$ such that

$$
\left|\left\langle u_{n} \mathscr{R} A u\right\rangle\right| \leqq \sqrt{n} \sum_{|m-n|=1} D^{e x}\left(u_{m}\right) .
$$

From (4.25), (4.26), (4.27), (4.30) and Proposition 4.11, by choosing $\varepsilon$ and $\delta$ sufficiently small we get the estimate (4.22).

\section{Step 2. Second a priori Estimate}

Theorem 4.12. For any $\lambda>0$ there is a constant $C_{g}$ such that the solution $u_{\lambda}$ of (4.21) satisfies the estimate

$$
\lambda^{2}\left\langle u_{\lambda},(-S)^{-1} u_{\lambda}\right\rangle_{0} \leqq C_{g}
$$

We first introduce some notation. Note that the operator $S$ restricted to a function of degree $n$ is the generator of symmetric random walks of $n$ particles with Neumann boundary conditions whenever two particles of the same velocity are next to each other. We now introduce a new generator $\tilde{S}$ representing the generator of symmetric simple random walks of $n$ particles. Explicitly, if we write

$$
u_{n}(\eta)=\sum_{x_{1}, \ldots, x_{n}} \sum_{v_{1}, \ldots, v_{n}} u\left(x_{1}, \ldots, x_{n} ; v_{1}, \ldots, v_{n}\right) \prod_{i=1}^{n}\left[\eta\left(x_{i}, v_{1}\right)-\vartheta\right]
$$

for some function $u\left(x_{1}, \ldots, x_{n} ; v_{1}, \ldots, v_{n}\right)$, the action of $\tilde{S}$ on $u_{n}$ corresponds to the action on $u\left(x_{1}, \ldots, x_{n} ; v_{1}, \ldots, v_{n}\right)$ of the discrete Laplacian on the coordinates $x_{1}, \ldots, x_{n}$.

The following corollary of Lemma 4.10 is proved in [15]:

Corollary 4.13. There exists a universal constant $C$ such that for any cylindric function $g$ of degree $n$

$$
\begin{aligned}
\langle g,(-S) g\rangle_{0} & \leqq\langle g,(-\tilde{S}) g\rangle_{0} \leqq C n\langle g,(-S) g\rangle_{0} \\
(C n)^{-1}\left\langle g,(-S)^{-1} g\right\rangle_{0} & \leqq\left\langle g,(-\tilde{S})^{-1} g\right\rangle_{0} \leqq\left\langle g,(-S)^{-1} g\right\rangle_{0} .
\end{aligned}
$$

Proof of Theorem 4.12. From the definition

$$
\left.(-\lambda+\tilde{S}+Q+A) u_{\lambda}=g+(\tilde{S}-S)\right) u_{\lambda}:=h .
$$

Since $(\tilde{S}-S) f(\eta)=0$ whenever $\eta$ is a configuration that no two particles are at distance one from each other, from Theorem 4.9 and Lemma 4.13 the $H_{-1}$ norm of $(\tilde{S}-S)) u_{\lambda}$ is bounded in the following sense:

$$
\|(\tilde{S}-S)) u_{\lambda} \|_{-1} \leqq C n^{-\alpha}
$$

for any integer $\alpha>0$. Note that from Corollary 4.13 , the $H_{-1}$ can be taken w.r.t. either $S$ or $\tilde{S}$.

If $u_{\lambda}$ is a solution of 4.21 , we have

$$
\left\langle(-\lambda+\tilde{S}+Q+A) u_{\lambda},(-\tilde{S})^{-1}(-\lambda+\tilde{S}+Q+A) u_{\lambda}\right\rangle_{0}=\left\langle h,(-S)^{-1} h\right\rangle_{0} \leqq C_{g},
$$


where we have used (4.32). Expanding the left-hand side we get

$$
\begin{aligned}
\left\langle h,(-\tilde{S})^{-1} h\right\rangle_{0}= & \lambda^{2}\left\langle u_{\lambda},(-\tilde{S})^{-1} u_{\lambda}\right\rangle_{0}+\left\langle\tilde{S} u_{\lambda},(-\tilde{S})^{-1} \tilde{S} u_{\lambda}\right\rangle_{0} \\
& +\left\langle[Q+A] u_{\lambda},(-\tilde{S})^{-1}[Q+A] u_{\lambda}\right\rangle_{0} \\
& +2\left\langle\tilde{S} u_{\lambda},(-\tilde{S})^{-1}[Q+A] u_{\lambda}\right\rangle_{0}-2 \lambda\left\langle u_{\lambda},(-\tilde{S})^{-1} \tilde{S} u_{\lambda}\right\rangle_{0} \\
& -2 \lambda\left\langle u_{\lambda},(-\tilde{S})^{-1}[Q+A] u_{\lambda}\right\rangle_{0} .
\end{aligned}
$$

But

$$
\left\langle\tilde{S} u_{\lambda},(-\tilde{S})^{-1} \tilde{S} u_{\lambda}\right\rangle_{0}=-\left\langle u_{\lambda}, \tilde{S} u_{\lambda}\right\rangle_{0}
$$

Moreover

$$
\left\langle u_{\lambda}, A u_{\lambda}\right\rangle_{0}=0
$$

hence

$$
\left\langle\tilde{S} u_{\lambda},(-\tilde{S})^{-1}[Q+A] u_{\lambda}\right\rangle_{0}=-\left\langle u_{\lambda}, Q u_{\lambda}\right\rangle_{0}
$$

Finally,

$$
\left\langle u_{\lambda},(-\tilde{S})^{-1} \tilde{S} u_{\lambda}\right\rangle_{0}=-\left\langle u_{\lambda}, u_{\lambda}\right\rangle_{0}
$$

Therefore

$$
\begin{aligned}
\left\langle g,(-\tilde{S})^{-1} g\right\rangle_{0}= & \lambda^{2}\left\langle u_{\lambda},(-\tilde{S})^{-1} u_{\lambda}\right\rangle_{0}-\left\langle u_{\lambda}, \tilde{S} u_{\lambda}\right\rangle_{0}+\left\langle[Q+A] u_{\lambda},(-\tilde{S})^{-1}[Q+A] u_{\lambda}\right\rangle_{0} \\
& -2\left\langle u_{\lambda}, Q u_{\lambda}\right\rangle_{0}+2 \lambda\left\langle u_{\lambda}, u_{\lambda}\right\rangle_{0}-2 \lambda\left\langle u_{\lambda},(-\tilde{S})^{-1}[Q+A] u_{\lambda}\right\rangle_{0} .
\end{aligned}
$$

All the terms in (4.34) are non-negative but the last one. We now bound the last term. It contains two contributions, one due to collisions and the other to the antisymmetric part of the generator. The second one is estimated in [15], where the following proposition is proved:

Proposition 4.14. There is a constant $C_{n}$, growing as a power of $n$, such that, for $\lambda>0$,

$$
\lambda\left\langle u_{\lambda,},(-\tilde{S})^{-1} A u_{\lambda}\right\rangle_{0} \leqq \frac{\lambda^{2}}{4} \sum_{n}\left\|\pi_{n} u_{\lambda}\right\|_{-1, e x}^{2}+\sum_{n} C_{n}\left\|\pi_{n} u\right\|_{1, e x}^{2},
$$

where

$$
\|u\|_{1, e x}^{2}=\langle u,(-\tilde{S}) u\rangle_{0}
$$

We now bound in a similar way the term involving collisions.

Proposition 4.15. There is a constant $C_{n}$, growing as a power of $n$, such that, for $\lambda>0$,

$$
2 \lambda\left\langle u_{\lambda},(-\tilde{S})^{-1} Q u_{\lambda}\right\rangle_{0} \leqq \frac{\lambda^{2}}{4} \sum_{n}\left\|\pi_{n} u_{\lambda}\right\|_{-1, e x}^{2}+\sum_{n} C_{n}\left\|\pi_{n} u_{\lambda}\right\|_{1, e x}^{2} .
$$


Proof. Write $Q=\sum_{x} Q_{x}$. By definition of $Q_{x}$ we have

$$
\begin{aligned}
& \lambda\left\langle u_{\lambda},(-\tilde{S})^{-1}, Q_{x} u_{\lambda}\right\rangle_{0}=-(1 / 2) \lambda\left\langle Q_{x}(-\tilde{S})^{-1} u_{\lambda}, Q_{x} u_{\lambda}\right\rangle_{0} \\
& \quad=-\lambda\left\langle\left[Q_{x},(-\tilde{S})^{-1}\right] u_{\lambda}, Q_{x} u_{\lambda}\right\rangle_{0}-(1 / 2) \lambda\left\langle Q_{x} u_{\lambda},(-\tilde{S})^{-1} Q_{x} u_{\lambda}\right\rangle_{0} .
\end{aligned}
$$

The last term is nonpositive and can be omitted to get an upper bound.

The commutator term is bounded as follows: the function $u\left(x_{1}, \ldots, x_{n} ; v_{1}, \ldots, v_{n}\right)$ is defined only when the hard core condition is satisfied. We set $u\left(x_{1}, \ldots, x_{n} ; v_{1}, \ldots\right.$, $\left.v_{n}\right)=0$ whenever the hard core condition is violated. For any nonpositive matrix $B$ on the velocity space let

$$
B_{j} u\left(x_{1}, \ldots, x_{n} ; v_{1}, \ldots, v_{n}\right)=\sum_{w \in \mathscr{V}} B\left(v_{j}, w\right) u\left(x_{1}, \ldots, x_{n} ; v_{1}, \ldots, v_{j-1}, w, v_{j+1}, \ldots, v_{n}\right)
$$

Note that since we have defined $u$ everywhere by setting $u=0$ on the undefined set, $B_{j}$ is well defined and independent of the position coordinates. From explicit computation there exist a nonpositive matrix $B$ and an operator $\omega_{x}$ with $\omega_{x} u=0$ unless $x_{i}=x_{j}=x$ for some $i \neq j$, i.e. there are two particles at the same site, such that $\left(Q_{x} u\right)=\sum_{j} B_{j} u+\omega_{x} u$. Note that $\omega_{x} u$ may change the degree of $u$.

By definition, $\tilde{S}$ is an operator independent of the velocity indices. Hence $\tilde{S}$ and $B$ commute and thus $[\tilde{S}, B]=0$. Hence

$$
\begin{aligned}
\lambda\left\langle\left[(-\tilde{S})^{-1}, Q_{x}\right] u_{\lambda}, Q_{x} u_{\lambda}\right\rangle_{0} & =\lambda\left\langle u_{\lambda},\left[-\tilde{S}^{-1}, \omega_{x}\right] Q_{x} u_{\lambda}\right\rangle_{0} \\
& =\lambda\left\langle u_{\lambda},\left((-\tilde{S})^{-1} \omega_{x}-\omega_{x}(-\tilde{S})^{-1}\right) Q_{x} u_{\lambda}\right\rangle_{0} \\
& :=\Omega_{1}+\Omega_{2} .
\end{aligned}
$$

Clearly, $\Omega_{1}^{x}$ and $\Omega_{2}^{x}$ are similar and we only bound $\Omega_{1}$. From the Schwartz inequality,

$$
\left|\Omega_{1}\right| \leqq\left(\lambda^{2} / 8\right)\left\langle u_{\lambda},(-\tilde{S})^{-1} u_{\lambda}\right\rangle_{0}+8\left\langle\omega_{x} Q_{x} u_{\lambda},(-\tilde{S})^{-1} \omega_{x} Q_{x} u_{\lambda}\right\rangle_{0}
$$

Let $\tilde{\omega}_{x}=\omega_{x} Q_{x}$. Clearly, $\tilde{\omega}_{x}$ vanishes unless there are at least two particles at the same site. Hence from Lemma 4.10 we have

$$
8\left\langle\omega_{x} Q_{x} u_{\lambda},(-\tilde{S})^{-1} \omega_{x} Q_{x} u_{\lambda}\right\rangle_{0} \leqq 8 \sum_{n} C_{n}\left\|\pi_{n} u_{\lambda}\right\|_{1, e x}^{2}
$$

for some constant growing with $n$ as power law. From (4.32), (4.36), (4.37), (4.38), (4.39), we conclude this lemma.

Returning to the proof of Theorem 4.12, we have thus proved that

$$
\left\langle g,(-\tilde{S})^{-1} g\right\rangle_{0} \geqq(1 / 2) \lambda^{2}\left\langle u_{\lambda},(-\tilde{S})^{-1} u_{\lambda}\right\rangle_{0}-\sum_{n} \sum_{|n-m| \leqq N} C_{m}\left\|u_{\lambda}^{(m)}\right\|_{1}^{2}
$$

Using the (4.22) to estimate $\left\|u_{\lambda}^{(n)}\right\|_{1}^{2}$, with $\beta$ sufficiently large, we have

$$
\lambda^{2}\left\langle u_{\lambda},(-\tilde{S})^{-1} u_{\lambda}\right\rangle_{0} \leqq C_{g}
$$

This concludes the second a priori estimate of Theorem 4.12. 
Remark. Of course the a priori estimates we proved are true also if the asymmetric part of the generator $A$ is absent. We are going to use them in this form in the next Step 3, to get the proof of Lemma 4.7. In the next section we will use them in the full form to get a new structure theorem based on the operator $\mathscr{L}$.

Step 3. This step is similar to the analogous one in [15].

We have constructed solutions to the equation $(-\lambda+S+\mathscr{R} Q) u_{\lambda}=g$ with $\left\|u_{\lambda}\right\|_{1}+\lambda\left\|u_{\lambda}\right\|_{-1, e x}$ uniformly bounded. Then $u_{\lambda}$ converges weakly to some $u_{0}$ in $H_{1}$ and $\lambda u_{\lambda}$ converges weakly to some $w_{0}$ in $H_{-1, e x}$. We claim that $w_{0}=0$. To prove this, we have for all functions $v \in H_{1} \cap H_{-1, e x}$,

$$
\left\langle v, u_{\lambda}\right\rangle \rightarrow\left\langle v, u_{0}\right\rangle ; \quad \lambda\left\langle v, u_{\lambda}\right\rangle \rightarrow\left\langle v, w_{0}\right\rangle .
$$

Hence

$$
\left\langle v, w_{0}\right\rangle=0,
$$

since $H_{1} \cap H_{-1, e x}$ is dense in $H_{1}$. We have thus proved that $w_{0}=0$ in $H_{-1, e x}$. Hence $\lambda u_{\lambda}$ converges weakly to 0 and thus $(S+\mathscr{R} Q) u_{\lambda}$ converges weakly to $g$ in $H_{-1 \text {,ex }}$. This implies that some linear combination of $(S+\mathscr{R} Q) u_{\lambda}$ converges to $g$ strongly in $H_{-1, e x}$. Hence for any $\varepsilon>0$ there exist constants $\alpha_{\lambda}$ equal to 0 for all but a finite number of $\lambda$ such that

$$
\left\|\sum_{\lambda} \alpha_{\lambda}(S+\mathscr{R} Q) u_{\lambda}-g\right\|_{-1, e x} \leqq \varepsilon .
$$

We put $u=\sum_{\lambda} \alpha_{\lambda} u_{\lambda}$. As a consequence, for any $\varepsilon>0$ there is $u$ such that

$$
\|(S+\mathscr{R} Q) u-g\|_{-1, e x} \leqq \varepsilon,
$$

and this concludes the proof of Lemma 4.7.

\section{Proof of Lemma 4.8 .}

Step 1. Since nondegenerate norms on $\mathscr{G}_{-}$are equivalent, we choose arbitrarily one of them and denote it by $\|\cdot\|$. Let $z=\sum_{\alpha<0} a_{\alpha} \tilde{I}_{\alpha}$ be any element of $\mathscr{G}_{-}$of norm one. By Lemma 4.7 , for any $\varepsilon>0$ there is $g \in \mathscr{R} \mathscr{G}$, such that

$$
\left\|\mathscr{R} \mathscr{L}_{s} z-\mathscr{R} \mathscr{L}_{s} g\right\|_{-1, e x}<\varepsilon .
$$

Let

$$
w=(1-\mathscr{R}) \mathscr{L}_{s}(z-g)
$$

be the projection onto degree one space. We claim that for any $z$ fixed,

$$
\|w\| \leqq C
$$

for some constant independent of $\varepsilon$. To prove it we take the inner product of $(4.40)$ by $w$ and use the fact that $w$ is of degree 1 to get

$$
\langle w, w\rangle_{0}=\left\langle w,(1-\mathscr{R}) \mathscr{L}_{s}(z-g)\right\rangle_{0}=\left\langle w, \mathscr{L}_{s}(z-g)\right\rangle_{0} .
$$

By definition, $\left\langle w, \mathscr{L}_{s}(z-g)\right\rangle_{0}=\left\langle\mathscr{L}_{s} w,(z-g)\right\rangle_{0}$. Since $w$ is a conserved quantity for the exclusion process, we have $\left\langle\mathscr{L}_{s} w,(z-g)\right\rangle_{0}=\langle Q w,(z-g)\rangle_{0}$. Hence

$$
\begin{aligned}
\left|\left\langle w, \mathscr{L}_{s}(z-g)\right\rangle_{0}\right| & =\left|\langle w, Q(z-g)\rangle_{0}\right| \\
& \leqq \delta\langle w,(-Q) w\rangle_{0}+\delta^{-1}\langle(z-g),(-Q)(z-g)\rangle_{0} .
\end{aligned}
$$


Hence

$$
\langle w, w\rangle_{0}-\delta\left|\langle w,(-Q) w\rangle_{0}\right| \leqq 2 \delta^{-1}\langle z,(-Q) z\rangle_{0}+2 \delta^{-1}\langle g,(-Q) g\rangle_{0}
$$

From Theorem 4.9, $\langle g,(-Q) g\rangle_{0}$ is uniformly bounded. By choosing $\delta$ small enough, we have $\langle w, w\rangle_{0}$ is uniformly bounded. For future purposes, we note also that this argument applies if $\mathscr{L}_{s}$ is replaced by $\mathscr{L}$.

Since $w$ is uniformly bounded, by letting $\varepsilon \rightarrow 0$ we can define a linear map from $\mathscr{G}_{-}$to itself. Denote this map by $\mathscr{U}$. In order to prove Lemma 4.8 it is sufficient to show that $\mathscr{U}$ is nonsingular.

Step 2. Suppose that $\mathscr{U}$ is singular. Then for any $\varepsilon>0$ there is $z$ of norm one and $g$ such that

$$
\left\|\mathscr{R} \mathscr{L}_{s}(z-g)\right\|_{-1, e x}<\varepsilon, \quad\left\|(1-\mathscr{R}) \mathscr{L}_{s}(z-g)\right\|<\varepsilon
$$

Note that these two bounds imply that

$$
\begin{aligned}
\left\|\mathscr{L}_{s}(z-g)\right\|_{-1} & \leqq\left\|\mathscr{R} \mathscr{L}_{s}(z-g)\right\|_{-1}+\left\|(1-\mathscr{R}) \mathscr{L}_{s}(z-g)\right\|_{-1} \\
& \leqq\left\|\mathscr{R} \mathscr{L}_{s}(z-g)\right\|_{-1, e x}+C\left\|(1-\mathscr{R}) \mathscr{L}_{s}(z-g)\right\| \leqq C \varepsilon,
\end{aligned}
$$

where we have used the triangular inequality for the norm \|\|$_{-1}$ and the equivalence of norms on $\mathscr{G}_{-}$.

Hence we have

$$
\langle[z-g],(-Q)[z-g]\rangle_{0} \leqq C \varepsilon .
$$

Again, the same estimate holds if $\mathscr{L}_{S}$ is replaced by $\mathscr{L}$.

Step 3. We claim that the last bound implies that $\|z\| \leqq C \varepsilon$. Once proved, the claim implies Lemma 4.8. In fact, assuming the bound, for $\varepsilon$ small enough we get contradiction with the assumption that $\|z\|=1$ and hence $\mathscr{U}$ is nonsingular.

We now prove the claim. From the definition of $\langle u,(-Q) u\rangle_{0}$ we have

$$
\langle u,(-Q) u\rangle_{0}=E\left[\sum_{x} \tau_{x} u(-Q) u\right]=\frac{1}{2} E\left[\left(Q_{0} \sum_{x} \tau_{x} u\right)^{2}\right]
$$

Hence

$$
\langle[z-g],(-Q)[z-g]\rangle_{0}=\frac{1}{2} E\left[Q_{0} \sum_{x} \tau_{x}(z-g)\right]^{2}=\frac{1}{2} E\left[Q_{0}\left(z-\sum_{x} \tau_{x} g\right)\right]^{2} .
$$

By convexity, we have for any local function $u, E\left\{Q_{0} E\left[u \mid \mathscr{F}_{0}\right]\right\}^{2} \leqq E\left[Q_{0} u\right]^{2}$, where $\mathscr{F}_{0}$ is the $\sigma$-algebra at the site 0 . Note that since $g$ is degree two or higher, so is $h=E\left(u \mid \mathscr{F}_{0}\right)$. Our goal is to prove that $E z^{2} \leqq C \varepsilon$ given that $E\left[Q_{0}(z-h)\right]^{2} \leqq C \varepsilon$ for some $h$ degree two or higher. This is an elementary estimate. Let $V$ denote the vector space of all measurable functions on the site 0 . Let $V^{+}$be the kernel of $Q_{0}$ consisting of functions depending only on $I_{0}^{+}$and $\pi_{+}$be the projection onto $V^{+}$with inner product $E[u ; v]$. Since $E[z ; \xi]=0$ for all $\xi \in V^{+}$we have $\pi^{+} z=0$. Therefore,

$$
E\left[Q_{0}\left(z-\left(1-\pi^{+}\right) h\right)\right]^{2}=E\left[Q_{0}\left(1-\pi^{+}\right)(z-h)\right]^{2}=E\left[Q_{0}(z-h)\right]^{2} \leqq C \varepsilon .
$$


(Since $Q_{0} \pi^{+}(z-h)=0$ by definition of $V^{+}$.) Because $z-\left(1-\pi^{+}\right) h$ is orthogonal to $V^{+}$, there is a uniform constant $\gamma$ such that

$$
E\left[\left(z-\left(1-\pi^{+}\right) h\right)\right]^{2} \leqq \gamma E\left[Q_{0}\left(z-\left(1-\pi^{+}\right) h\right)\right]^{2} .
$$

Since $h$ is degree two or higher,

$$
E z^{2} \leqq E\left[\left(z-\left(1-\pi^{+}\right) h\right)\right]^{2} \leqq \gamma C \varepsilon .
$$

This proves the bound and concludes the proof of Lemma 4.8.

\section{The Diffusion Coefficient}

The space $\overline{\mathscr{G}}$, (see Definition 4.1 and Theorem 4.2) with the scalar product (4.7), is a Hilbert space and it can be decomposed into $\mathscr{G}^{0}$ and $\mathscr{L}_{s} \mathscr{G}$ (4.8). The structure theorem as well as the scalar product $\langle\langle\cdot, \cdot\rangle\rangle$ are based on the symmetric part of the generator, $\mathscr{L}_{s}$. The strategy of the non-gradient method, introduced for the first time by Varadhan in [16] is to decompose the currents into the sum of a gradient term and a term of the form $\mathscr{L} \phi$. We want to adopt a similar strategy. To do this we need a new structure theorem based on the complete generator $\mathscr{L}$. We get such a theorem following the procedure already used in [8 and 15]. As a consequence we identify the diffusion coefficient and characterize it by a variational principle. We will use also in this section the indices $\alpha, \beta, \gamma=0, \ldots, d$ to denote the "color" indices, while latin indices $i, j, k=1, \ldots, d$ will denote the $d$ coordinate directions. We recall the notation:

$$
\nabla_{j} g(\eta)=g\left(\tau_{e_{j}} \eta\right)-g(\eta)
$$

where $\left(\tau_{x} \eta\right)(y, v)=\eta(y+x, v)$ and $\left(\tau_{x} f\right)(\eta)=f\left(\tau_{x} \eta\right)$. This should not be confused with the other notation we use:

$$
\nabla_{v, j} g(\eta)=g\left(\eta^{0, e_{j}, v}\right)-g(\eta)
$$

For any local function $g$, we set

$$
t_{j}^{\alpha}(g)=\frac{1}{\chi} \lim _{l \rightarrow \infty} E^{\mu}\left[g \sum_{x \in \Lambda_{l}}\left(e_{j} \cdot x\right) \tilde{I}_{\alpha}\left(\eta_{x}\right)\right] .
$$

Here $\tilde{I}$ and $\tilde{\eta}$ denote the centered variables introduced in (4.4). Note that the sum in the right-hand side of (5.1) is independent of $\ell$, for $\ell$ large enough, since $E^{\mu}\left[g I_{\alpha}\left(\eta_{x}\right)\right]=0$ if $g$ does not depend on $\eta_{x}$. Recall that for any functions $f$ and $g$,

$$
\langle f, g\rangle_{0}=\sum_{x}\left\langle f \tau_{x} g\right\rangle, \quad\left\langle\nabla_{j} I_{\alpha}\left(\eta_{0}\right), g\right\rangle_{0}=0, \quad \alpha \geqq 0, j=1, \ldots, d .
$$

It is often convenient to replace the space $\mathscr{G}$ with a different dense subset of $\overline{\mathscr{G}}$, namely the space $\mathscr{G}^{c}$ defined as follows:

Definition 5.1. Let

$$
\begin{aligned}
\mathscr{G}^{c}= & \left\{g \text { local function of } \eta: E\left[g \mid \bar{I}_{k}^{+}\right]=0 \text { for some } k>0\right. \\
& \text { and for all values of } \left.\bar{I}_{k}, \alpha \geqq 0\right\} .
\end{aligned}
$$

Here $E^{\mu}\left[\cdot \mid \bar{I}_{k}^{+}\right]$means expectation conditioned to fixed values of $A v_{x \in \Lambda_{k}} I_{\alpha}\left(\eta_{x}\right)$ for $\alpha=0, \ldots, d$. 
We claim that $\overline{\mathscr{G} c}=\overline{\mathscr{G}}$. To prove this, suppose that $g \in \mathscr{G}$. For any $k$ fixed and large enough, we have $g-g_{k} \in \mathscr{G}^{c}$, where $g_{k}=E^{\mu}\left[g \mid \bar{I}_{k}^{+}\right]$. Also, by definition, $g_{k} \in \mathscr{G}$. One can easily prove that $\left\|g_{k}\right\|_{-1}$ is uniformly bounded. Clearly, by the equivalence of ensembles we have $g_{k} \rightarrow E[g]=0$. This proves our claim.

The following lemma provides the expression of the scalar product $\langle\langle\cdot, \cdot\rangle\rangle$ on $\mathscr{G}^{c}$.

Lemma 5.2. The scalar product $\langle\langle\cdot, \cdot\rangle\rangle$ defined by (4.7) is such that for any $g, h \in \mathscr{G}^{c}:$

$$
\begin{gathered}
\left\langle\left\langle\mathscr{L}_{s} g, h\right\rangle=-\langle h, g\rangle_{0},\right. \\
\mathscr{G}^{0} \perp \mathscr{L}_{s} \mathscr{G}, \\
\left\langle\left\langle\mathscr{L}_{s} g, \mathscr{L}_{s} h\right\rangle=\frac{1}{2} E^{\mu}\left[\sum_{v \in \mathscr{V}} \sum_{j=1}^{d} \chi\left(\nabla_{v, j} \sum_{x} \tau_{x} g\right)\left(\nabla_{v, j} \sum_{x} \tau_{x} h\right)\right.\right. \\
\left.+\sum_{q \in \mathscr{Q}}\left(Q_{0}^{q} \sum_{x} \tau_{x} g\right)\left(Q_{0}^{q} \sum_{x} \tau_{x} h\right)\right], \\
\left\langle\left\langle\nabla_{j} I_{\alpha}\left(\eta_{0}\right), g\right\rangle\right\rangle=t_{j}^{\alpha}(g), \\
\left\langle\left\langle\nabla_{i} I_{\alpha}(\eta), \nabla_{j} I_{\beta}\left(\eta_{0}\right)\right\rangle\right\rangle=t_{i}^{\alpha}\left(\nabla_{j} I_{\beta}(\eta)\right)=\frac{1}{2 \chi} \delta_{i, j} \delta_{\alpha, \beta} E^{\mu}\left[\left(\nabla_{i} I_{\alpha}\left(\eta_{0}\right)\right)^{2}\right] .
\end{gathered}
$$

Proof. Equation (5.3) follows from the definition of the scalar product and the arguments used in the proof of Theorem 4.4. The orthogonality relation (5.4) follows by using (5.3) to get

$$
\left\langle\left\langle\mathscr{L}_{s} g, \nabla_{j} I_{\alpha}\left(\eta_{0}\right)\right\rangle\right\rangle=-\left\langle\nabla_{j} I_{\alpha}\left(\eta_{0}\right), g\right\rangle_{0}=0, \quad \alpha \geqq 0,
$$

the last step being a consequence of the fact that $\mathscr{G}^{0}$ is in the null space of the inner product $\langle\cdot, \cdot\rangle_{0}$. Again using the definition of the scalar product and the structure of $\mathscr{L}_{S}$, we get (5.5). Relations (5.6) and (5.7) are consequence of the definition of the scalar product and of the relation

$$
\mathscr{L}_{s} \sum_{x}\left(e_{j} \cdot x\right) I_{\alpha}\left(\eta_{x}\right)=-\chi \nabla_{j} I_{\alpha}\left(\eta_{0}\right)
$$

Finally, (5.7) follows from explicit computation.

We have the following variational formula for the variance $V$.

Theorem 5.3. For any $g \in \mathscr{G}$ the variance $V(g):=\langle\langle g, g\rangle\rangle$ satisfies the variational principle

$$
\begin{aligned}
\frac{1}{2} V(g)= & \sup _{c_{j}^{\alpha} \in \mathbb{R}, h \in \mathscr{G} c}\left[\sum_{\alpha \geqq 0, j \geqq 1} c_{j}^{\alpha} t_{j}^{\alpha}(g)+\langle g, h\rangle_{0}-\frac{1}{4}\left\{\sum_{\alpha \geqq 0, j \geqq 1}\left(c_{j}^{\alpha}\right)^{2} V\left(\nabla_{j} I_{\alpha}(\eta)\right)\right.\right. \\
& \left.\left.+E^{\mu}\left[\sum_{v \in \mathscr{V}, j \geqq 1} \chi\left(\nabla_{v, j} \sum_{x} \tau_{x} h\right)^{2}+\sum_{q \in \mathscr{Q}}\left(Q_{0}^{q} \sum_{x} \tau_{x} h\right)^{2}\right]\right\}\right] .
\end{aligned}
$$


Proof. For $g \in \mathscr{G}$ the following identity holds:

$$
\frac{1}{2}\langle\langle g, g\rangle\rangle=\sup _{u \in \mathscr{G} c}\left\{\langle\langle g, u\rangle\rangle-\frac{1}{2}\langle\langle u, u\rangle\rangle\right\}
$$

By the structure theorem, Theorem 4.2, for any $\varepsilon>0$ there exist a set $\left\{c_{j}^{\alpha}\right\}$ and $h \in \mathscr{G}$ such that

$$
\left\|u-\sum_{\alpha \geqq 0} \sum_{j=1}^{d} c_{j}^{\alpha} \nabla_{j} I_{\alpha}+\mathscr{L}_{s} h\right\|_{-1}<\varepsilon .
$$

Hence, since $\varepsilon$ is arbitrary, we get

$$
\begin{aligned}
\frac{1}{2}\langle\langle g, g\rangle\rangle= & \sup _{c_{j}^{\alpha}, h \in \mathscr{G}_{c}}\left\{\sum_{\alpha \geqq 0, j \geqq 1} c_{j}^{\alpha}\left\langle\left\langle g, \nabla_{j} I_{\alpha}\right\rangle\right\rangle+\left\langle\left\langle g, \mathscr{L}_{s} h\right\rangle\right\rangle\right. \\
& \left.-\frac{1}{2}\left[\sum_{\alpha \geqq 0, j \geqq 1} \sum_{\beta \geqq 0, i \geqq 1} c_{j}^{\alpha} c_{i}^{\beta}\left\langle\left\langle\nabla_{j} I_{\alpha}, \nabla_{i} I_{\beta}\right\rangle\right\rangle+\left\langle\left\langle\mathscr{L}_{s} h, \mathscr{L}_{s} h\right\rangle\right\rangle\right]\right\} .
\end{aligned}
$$

Finally, using Lemma 5.2 in (5.10) we get the result.

We need another structure theorem for $\bar{G}$ so that its elements can be decomposed in terms of a gradient and $\mathscr{L} \mathscr{G}$. Theorem 5.5 below provides such a decomposition. First of all note that the following inclusion relations hold:

$$
\mathscr{L} \mathscr{G} \subset \mathscr{G}, \quad \mathscr{L}^{*} \mathscr{G} \subset \mathscr{G} .
$$

Recall the expressions (2.20) and (2.22) of the currents $w_{\alpha}^{\beta}$ (we set $x=0$ and omit the position label) given by

$$
\begin{aligned}
& w_{j}^{0}=\sum_{v \in \mathscr{V}} w_{j}(v)=w_{j}^{(s) 0}+\sum_{v \in \mathscr{V}}\left(e_{j} \cdot v\right) b_{j}(v), \\
& w_{j}^{\alpha}=\sum_{v \in \mathscr{V}}\left(e_{\alpha} \cdot v\right) w_{j}(v)=w_{j}^{(s) \alpha}+\sum_{v \in \mathscr{V}}\left(e_{\alpha} \cdot v\right)\left(e_{j} \cdot v\right) b_{j}(v), \quad \alpha=1, \ldots, d,
\end{aligned}
$$

with

$$
w_{j}^{(s) \alpha}=\chi \nabla_{j} I_{\alpha}\left(\eta_{0}\right), \quad b_{j}(v)=\eta\left(e_{j}, v\right) \eta(0, v)-\frac{1}{2}\left[\eta\left(e_{j}, v\right)+\eta(0, v)\right] .
$$

Note that $w_{j}^{\alpha} \notin \mathscr{G}$. It is obvious that there exist constants $c_{i}^{\alpha, \beta}$ such that

$$
\sigma_{i}^{\alpha}=w_{i}^{\alpha}-\sum_{\beta \geqq 0} c_{i}^{\alpha, \beta} \tilde{I}_{\beta}\left(\eta_{0}\right) \in \tilde{\mathscr{G}}
$$

The explicit form of $c_{i}^{\alpha, \beta}$ is not important and all the computations in this section are independent of $c_{i}^{\alpha, \beta}$. In the same way, one can define $\sigma_{i}^{* \alpha}=w_{i}^{* \alpha}-$ $\sum_{\beta \geqq 0} c_{i}^{* \alpha, \beta} \tilde{I}_{\beta}\left(\eta_{0}\right) \in \tilde{\mathscr{G}}$. Note that from (5.3), for $g \in \mathscr{G},\left\langle g, w_{i}^{\beta}\right\rangle_{0}=\left\langle g, \sigma_{i}^{\beta}\right\rangle_{0}$ and $\left\langle g, w_{i}^{* \beta}\right\rangle_{0}=\left\langle g, \sigma_{i}^{* \beta}\right\rangle_{0}$. 
Lemma 5.4. Suppose $g \in \mathscr{G}$. Then for $\alpha=0, \ldots, d$ and $j=1, \ldots, d$,

$$
\begin{gathered}
\chi t_{j}^{\alpha}(\mathscr{L} g)=-\left\langle g, w_{j}^{* \alpha}\right\rangle_{0}=-\left\langle g, \sigma_{j}^{* \alpha}\right\rangle_{0}, \\
\chi t_{j}^{\alpha}\left(\mathscr{L}^{*} g\right)=-\left\langle g, w_{j}^{\alpha}\right\rangle_{0}=-\left\langle g, \sigma_{j}^{\alpha}\right\rangle_{0}, \\
\left\langle\left\langle\sigma_{j}^{\beta}, \nabla_{i} I_{\alpha}(\eta)\right\rangle\right\rangle=t_{i}^{\alpha}\left(\sigma_{j}^{\beta}\right)=\delta_{i, j} \delta_{\alpha, \beta} \chi V\left(\nabla_{i} I_{\alpha}\right), \quad \alpha, \beta, i, j=1, \ldots, d . \\
\left\langle\left\langle\sigma_{i}^{0}, \nabla_{j} I_{0}(\eta)\right\rangle\right\rangle=\delta_{i, j} \chi V\left(\nabla_{i} I_{0}\right), \quad i, j=1, \ldots, d .
\end{gathered}
$$

Proof. The relations (5.13) and (5.14) follow from the identities

$$
\mathscr{L}^{*} \sum_{x}\left(x \cdot e_{j}\right) \tilde{I}_{\alpha}(\eta(x))=-\sum_{x} w_{j, x}^{* \alpha}, \quad \mathscr{L} \sum_{x}\left(x \cdot e_{j}\right) \tilde{I}_{\alpha}(\eta(x))=-\sum_{x} w_{j, x}^{\alpha},
$$

and the same argument used in the proof of Lemma 5.7 in [8]. To prove (5.15), from (5.1) and (5.16) and the definition of $\sigma_{j}^{\beta}$ we get

$$
\begin{aligned}
\chi t_{i}^{\alpha}\left(\sigma_{j}^{\beta}\right) & =\lim _{\ell \rightarrow \infty} E^{\mu}\left[\sigma_{j}^{\beta} \sum_{|x| \leqq \ell}\left(x \cdot e_{i}\right) \tilde{I}_{\alpha}(\eta(x))\right] \\
& =\lim _{\ell \rightarrow \infty} E^{\mu}\left[w_{j}^{\beta} ; \sum_{|x| \leqq \ell}\left(x \cdot e_{i}\right) \tilde{I}_{\alpha}(\eta(x))\right] \\
& =\lim _{\ell \rightarrow \infty} \ell^{-d} E^{\mu}\left[\mathscr{L} \sum_{x}\left(x \cdot e_{j}\right) \tilde{I}_{\alpha}(\eta(x)) ; \sum_{|x| \leqq \ell}\left(x \cdot e_{i}\right) \tilde{I}_{\alpha}(\eta(x))\right] .
\end{aligned}
$$

Clearly, if $j \neq i$ or $\alpha \neq \beta$, then $t_{i}^{\alpha}\left(\sigma_{j}^{\beta}\right)=0$ by the reflection symmetry. Hence we assume $i=j$ and $\alpha=\beta$. But under this assumption we can replace $\mathscr{L}$ by $\mathscr{L}_{s}$. On the other hand, from (5.6) and (5.7),

$\lim _{\ell \rightarrow \infty} \ell^{-d} E^{\mu}\left[\mathscr{L} \sum_{x}\left(x \cdot e_{i}\right) \tilde{I}_{\alpha}(\eta(x)) ; \sum_{|x| \leqq \ell}\left(x \cdot e_{i}\right) \tilde{I}_{\alpha}(\eta(x))\right]=\chi^{2} t_{i}^{\alpha}\left(\nabla_{i} I_{\alpha}\right)=\chi^{2} V\left(\nabla_{i} I_{\alpha}\right)$

This proves Lemma 5.4.

Next we state the second structure theorem for $\overline{\mathscr{G}}$.

\section{Theorem 5.5.}

(i) $\overline{\mathscr{L} \mathscr{G}+\mathscr{G}_{0}}=\overline{\mathscr{G}}=\overline{\mathscr{L} * \mathscr{G}+\mathscr{G}_{0}}$.

(ii) Let $\mathscr{G}_{w}=\left\{\sum_{\alpha \geqq 0, j \geqq 1} b_{j}^{\alpha} \sigma_{j}^{\alpha}\right\}$. Then

$$
\overline{\mathscr{G}_{w}+\mathscr{L}_{s} \mathscr{G}}=\overline{\mathscr{G}}=\overline{\mathscr{G}_{w}+\mathscr{L} \mathscr{G}} .
$$

Furthermore,

$$
V\left(\sum_{\alpha \geqq 0, j \geqq 1} b_{j}^{\alpha} \sigma_{j}^{\alpha}+\mathscr{L} g\right) \geqq V\left(\sum_{\alpha \geqq 0, j \geqq 1} b_{j}^{\alpha} w_{j}^{(s) \alpha}+\mathscr{L}_{s} g\right) .
$$


Similarly, $\overline{\mathscr{G}_{w^{*}}+\mathscr{L}^{* \mathscr{G}}}=\overline{\mathscr{G}}$ and

$$
V\left(\sum_{\alpha \geqq 0, j \geqq 1} b_{j}^{\alpha} \sigma_{j}^{* \alpha}+\mathscr{L}^{*} g\right) \geqq V\left(\sum_{\alpha \geqq 0, j \geqq 1} b_{j}^{\alpha} w_{j}^{(s) \alpha}+\mathscr{L}_{s} g\right) .
$$

Proof of $(i)$. All the ingredients of the proof have been already presented in Sect. 4. In fact, suppose first that $g$ is of degree 2, namely $g \in \mathscr{R} \mathscr{G}$. Under this assumption the analog of Lemma 4.7 hold:

Lemma 5.6. For any local function $g \in \mathscr{R} \mathscr{G}$ and any $\varepsilon>0$ there is a local function $u \in \mathscr{G}$ such that

$$
\|\mathscr{R} \mathscr{L} u-g\|_{-1, e x} \leqq \varepsilon .
$$

The same statement is true with $\mathscr{L}$ replaced by $\mathscr{L}^{*}$.

Proof. The lemma follows from the same three steps of the proof of Lemma 4.7. The first two steps are already given in Sect. 4 in a form which includes the present case (Theorems 4.9 and 4.12 ). They provide a priori estimates for the solution $u_{\lambda}$ of (4.21). The third step is to show that we can find the function $u$ as the limit as $\lambda$ goes to 0 of $u_{\lambda}$. The argument in Sect. 4 is easily adapted to the present context because the norm $\|\cdot\|_{-1}$ is bounded by $\|\cdot\|_{-1, \text { ex }}$ according to (4.12). This concludes the proof of the Lemma 5.6.

To conclude the proof of (i) we have to show it for $g$ of degree 1, namely $g \in \mathscr{G}_{-}$. This is the analog of Lemma 4.8. Once the previous Lemma 5.6 is proved, as we already pointed out, the proof of Lemma 4.8 holds also with $\mathscr{L}_{s}$ replaced by $\mathscr{L}$. This concludes the proof of i).

Proof of (ii). Let $q \in \mathscr{G}_{w}$. By Theorem 4.2 for any $\varepsilon>0$, there are $v_{\varepsilon} \in \mathscr{G}^{0}$ and $u \in \mathscr{G}$ such that

$$
\left\|q-v_{\varepsilon}-\mathscr{L}_{s} u\right\|_{-1}<\varepsilon .
$$

Since $v_{\varepsilon} \perp \mathscr{L}_{s} u,\left\|v_{\varepsilon}\right\|_{-1}$ is uniformly bounded and we can take the limit $\varepsilon \rightarrow 0$ of the mapping $q \rightarrow v_{\varepsilon}$ to define a map $\mathscr{T}$. We claim that $\mathscr{T}$ is an invertible transformation from $\mathscr{G}_{w}$ to $\mathscr{G}^{0}$. In fact, since these two spaces have the same dimension, it is enough to show that $\mathscr{T}$ is not degenerate. Suppose on the contrary that there is $\bar{q} \in \mathscr{G}_{w}$ such that $\mathscr{T} \bar{q}=0$, i.e. for any $\varepsilon>0$ there is a $g \in \mathscr{G}$ such that

$$
\left\|\bar{q}-\mathscr{L}_{s} g\right\|_{-1}<\varepsilon .
$$

Suppose $\bar{q}=\sum_{\alpha, j} q_{j}^{\alpha} \sigma_{j}^{\alpha}$. Then, choose $p \in \mathscr{G}^{0}$ such that $p=\sum_{\alpha, j} q_{j}^{\alpha} \nabla_{j} I^{\alpha}$. We have

$$
|\langle\langle\bar{q}, p\rangle\rangle|=\left|\left\langle\bar{q}-\mathscr{L}_{s} g, p\right\rangle\right\rangle \mid \leqq \varepsilon\|p\|_{-1} .
$$

By (5.15) of Lemma 5.4, there is a constant $c$ such that

$$
|\langle\langle\bar{q}, p\rangle\rangle| \geqq c\|p\|_{-1}\|\bar{q}\|_{-1} .
$$

Combining the last two inequalities and choosing $\varepsilon$ arbitrarily small, we have $\bar{q}=0$. This proves the invertibility of $\mathscr{T}$, which implies immediately the first of (5.17). The second of (5.17) follows from this and the application of i) to $\mathscr{L}_{s} \mathscr{G}$. This proves (ii). 
We now prove the bound (5.18) on $V$. By Theorem 5.3 the left side of (5.18) is equal to

$$
\begin{aligned}
& 2 \sup _{\tilde{b}, h}\left\{\sum_{\alpha \geqq 0, i \geqq 1} \tilde{b}_{i}^{\alpha} t_{i}^{\alpha}\left(\sum_{\beta \geqq 0, j \geqq 1} b_{j}^{\beta} \sigma_{j}^{\beta}+\mathscr{L} g\right)+\left\langle\sum_{\beta \geqq 0, j \geqq 1}^{d} b_{k}^{\beta} \sigma_{k}^{\beta}+\mathscr{L} g, h\right\rangle_{0}\right. \\
& \left.-\frac{1}{4} E^{\mu}\left[\sum_{\alpha \geqq 0, i \geqq 1} \frac{\tilde{b}_{i}^{\alpha}}{\chi}\left(\nabla_{i} I_{\alpha}(\eta)\right)^{2}+\sum_{v \in \mathscr{V}, i \geqq 1} \chi\left(\nabla_{v, i} \sum_{x} \tau_{x} h\right)^{2}+\sum_{q \in \mathscr{Q}}\left(Q_{0}^{q} \sum_{x} \tau_{x} h\right)^{2}\right]\right\} .
\end{aligned}
$$

In particular, let $h=-g$ and $\tilde{b}_{i}^{\alpha}=b_{i}^{\alpha} \chi$. Hence from Lemma 5.1 we can bound the last expression from below by

$$
\begin{aligned}
& 2\left\{\sum_{\beta \geqq 0, l \geqq 1} \chi b_{i}^{\beta} t_{i}^{\beta}\left(\sum_{j \geqq 1, \gamma \geqq 0} b_{j}^{\gamma} \sigma_{j}^{\gamma}+\mathscr{L} g\right)-\left\langle\sum_{j \geqq 1, \gamma \geqq 0} b_{j}^{\gamma} \sigma_{j}^{\gamma}+\mathscr{L} g, g\right\rangle_{0}\right. \\
& \left.\quad-\frac{1}{2} V\left(\sum_{j \geqq 1, \gamma \geqq 0} b_{j}^{\gamma} \nabla_{j} I_{\gamma}(\eta)+\mathscr{L}_{s} g\right)\right\} .
\end{aligned}
$$

By definition of $\mathscr{L},\langle\mathscr{L} g, g\rangle_{0}=\left\langle\mathscr{L}_{s} g, g\right\rangle_{0}$. Also, from (5.13) $t_{j}^{\gamma}(\mathscr{L} g)=-\left\langle g, w_{j}^{* \gamma}\right\rangle_{0} \chi$. To summarize, we have

$$
\begin{aligned}
& \frac{1}{2} V\left(\sum_{j \geqq 1, \gamma \geqq 0} b_{j}^{\gamma} \sigma_{j}^{\gamma}+\mathscr{L} g\right) \geqq \sum_{\beta \geqq 0, i \geqq 1} \chi\left[b_{i}^{\beta} t_{i}^{\beta}\left(\sum_{j \geqq 1, \gamma \geqq 0} b_{j}^{\gamma} \sigma_{j}^{\gamma}\right)-\left\langle g, w_{i}^{* \beta}\right\rangle_{0}\right] \\
& -\left\langle\sum_{j \geqq 1, \gamma \geqq 0} b_{j}^{\gamma} \sigma_{j}^{\gamma}, g\right\rangle_{0}-\left\langle\mathscr{L}_{s} g, g\right\rangle_{0}-\frac{1}{2} V\left(\sum_{j \geqq 1, \gamma \geqq 0} b_{j}^{\gamma} \nabla_{j} I_{\gamma}(\eta)+\mathscr{L}_{s} g\right) .
\end{aligned}
$$

Since $g \in \mathscr{G},\left\langle\sigma_{j}^{\beta}, g\right\rangle_{0}=\left\langle w_{j}^{\beta}, g\right\rangle_{0}$ by (5.14). By definition $w_{j}^{\beta}+w_{j}^{\beta *}=2 w_{j}^{(s) \beta}$, and hence $\left\langle g, w_{j}^{\beta *}+w_{j}^{\beta}\right\rangle_{0}=0$. Together with (5.15)

$$
\begin{aligned}
& \frac{1}{2} V\left(\sum_{j \geqq 1, \beta \geqq 0} b_{j}^{\beta} \sigma_{j}^{\beta}+\mathscr{L} g\right) \\
& \quad \geqq V\left(\sum_{j \geqq 1, \beta \geqq 0} b_{j}^{\beta} w_{j}^{(s) \beta}\right)-\left\langle\mathscr{L}_{s} g, g\right\rangle_{0}-\frac{1}{2} V\left(\sum_{j \geqq 1, \beta \geqq 0} b_{j}^{\beta} w_{j}^{(s) \beta}+\mathscr{L}_{s} g\right) \\
& \quad=\frac{1}{2} V\left(\sum_{j \geqq 1, \beta \geqq 0} b_{j}^{\beta} w_{j}^{(s) \beta}+\mathscr{L}_{s} g\right) .
\end{aligned}
$$

This proves the bound on $V$ and concludes Theorem 5.5.

To use this decomposition of the Hilbert space, one should introduce a scalar product based on the inverse generator $\mathscr{L}^{-1}$ instead of using $\langle\langle\cdot, \cdot\rangle\rangle$ in which only the symmetric part appears. Unfortunately, we do not know how to give sense to $\mathscr{L}^{-1}$, so the arguments based on it are only formal and we have to introduce a suitable map $T$ below to make the computation rigorous. At a formal level one 
can think of the map $T$ as $T=\mathscr{L}_{s} \mathscr{L}^{-1}$, so that formally $\langle\langle g, T h\rangle\rangle=\left\langle g, \mathscr{L}^{-1} h\right\rangle_{0}$. With this in mind, it is easy to understand the statements of Theorems 5.7 and 5.8 below and the calculus of the diffusion matrix.

\section{Theorem 5.7.}

(i) Let

$$
u_{j}^{\beta}=\chi \nabla_{j} I_{\beta} .
$$

Define $T$ to be the linear transformation from $\mathscr{G}$ to $\mathscr{G}$ s.t.

$$
T\left(\sum_{j \geqq 1, \beta \geqq 0} b_{j}^{\beta} \sigma_{j}^{\beta}+\mathscr{L} g\right)=\sum_{j \geqq 1, \beta \geqq 0} b_{j}^{\beta} u_{j}^{\beta}+\mathscr{L}_{s} g .
$$

Then $T$ is bounded above by 1 , hence can be extended by continuity to $\overline{\mathscr{G}}$. Moreover

$$
\begin{aligned}
& \text { (a) } T \nabla_{j} I_{\alpha}(\eta) \perp \mathscr{L}^{*} \mathscr{G} \text { for } j \geqq 1, \alpha \geqq 0, \\
& \text { (b) }\left\langle\left\langle T \nabla_{i} I_{\alpha}(\eta), \sigma_{j}^{* \beta}\right\rangle\right\rangle=\chi \delta_{i, j} \delta_{\alpha, \beta} V\left(\nabla_{i} I_{\alpha}\right) .
\end{aligned}
$$

(ii) Let $M$ be the matrix (with double indices $(\alpha, i)$ and $(\beta, j)$ )

$$
M_{i, j}^{\alpha, \beta}=\left\langle\left\langle\nabla_{i} I_{\alpha}(\eta), T \nabla_{j} I_{\beta}(\eta)\right\rangle\right\rangle
$$

Then the diffusion matrix $D$ is given by

$$
D_{i, j}^{\alpha, \beta}=\chi\left(M^{-1}\right)_{l, j}^{\alpha, \beta} V\left(\nabla_{i} I_{\alpha}\right) .
$$

As a quadratic form, $D \geqq D_{s}$ and $D \neq D_{s}$, where $\left(D_{s}\right)_{i, j}^{\alpha, \beta}=\chi \delta_{\alpha, \beta} \delta_{i, j}$. Furthermore, there exist constants $D_{0}, D_{1}, D_{2}, D_{3}$ and $D_{4}$ such that

$$
\left.D_{j, k}^{\alpha, \beta}=\delta_{j, k} \delta_{\alpha, \beta}\left[D_{0} \delta_{\alpha, 0}+D_{1}\left(1-\delta_{\alpha, 0}\right)+D_{2} \delta_{\alpha, j}\right)\right]+D_{3} \delta_{\alpha, j} \delta_{\beta, k}+D_{4} \delta_{j, \beta} \delta_{i, k} .
$$

(iii) Since $T$ is one-to-one and the image is dense, its inverse $T^{-1}$ is well-defined on a dense subset of $\overline{\mathscr{G}}$. For any $\xi$ in the domain of $T^{-1}$,

$$
\left\langle\left\langle\xi,\left(T^{-1}\right)_{s} \xi\right\rangle\right\rangle=\left\langle\left\langle\xi, T^{-1} \xi\right\rangle\right\rangle=\langle\langle\xi, \xi\rangle\rangle .
$$

A similar statement holds with $T$ replaced by $T^{*}$.

Proof of $(i)$. The inequality (5.18) is precisely the statement that the map $T$ in (i) is bounded by 1 . To prove (a), recall that $\nabla_{i} I_{\alpha}$ as an element of $\mathscr{G}$ can be written as $\sum_{\beta \geqq 0, j \geqq 1} a_{i, j}^{\alpha \beta} \sigma_{j}^{\beta}+\mathscr{L} g$ with $g \in \mathscr{G}$ (approximation arguments are needed here and in the following). Then, by the definition of the map $T$,

$$
\left\langle\left\langle T \nabla_{i} I_{\alpha}, \mathscr{L}^{*} h\right\rangle\right\rangle=\sum_{\beta \geqq 0, j \geqq 1} a_{i, j}^{\alpha \beta}\left\langle\left\langle u_{j}^{\beta}, \mathscr{L}^{*} h\right\rangle\right\rangle+\left\langle\left\langle\mathscr{L}_{s} g, \mathscr{L}^{*} h\right\rangle\right\rangle .
$$

From Lemma 5.2 and (5.14) we get

$$
\left\langle\left\langle\mathscr{L}_{s} g, \mathscr{L}^{*} h\right\rangle\right\rangle=-\langle\mathscr{L} g, h\rangle_{0}, \quad\left\langle\left\langle u_{j}^{\beta}, \mathscr{L}^{*} h\right\rangle\right\rangle=-\left\langle\sigma_{j}^{\beta}, h\right\rangle_{0} .
$$


Then

$$
\left\langle\left\langle T \nabla_{i} I_{\alpha}, \mathscr{L}^{*} h\right\rangle\right\rangle=\left\langle\nabla_{i} I_{\alpha}, h\right\rangle_{0}=0 .
$$

To prove (b) of (i) we observe that, again by definition of $T$,

$$
\left\langle\left\langle T \nabla_{i} I_{\alpha}, \sigma_{k}^{* \gamma}\right\rangle\right\rangle=\left\langle\left\langle\sum_{\beta \geqq 0, j \geqq 1} a_{i, j}^{\alpha \beta} u_{j}^{\beta}, \sigma_{k}^{* \gamma}\right\rangle\right\rangle+\left\langle\left\langle\mathscr{L}_{s} g, \sigma_{k}^{* \gamma}\right\rangle\right\rangle .
$$

By Lemma 5.2 and Lemma 5.4 we have,

$$
\left\langle\left\langle\mathscr{L}_{s} g, \sigma_{k}^{* \gamma}\right\rangle\right\rangle=\left\langle g, \sigma_{k}^{* \gamma}\right\rangle_{0}=E\left[\mathscr{L} g \sum_{x}\left(x \cdot e_{k}\right) \tilde{I}_{\gamma}(\eta(x))\right]=\left\langle\left\langle\mathscr{L} g, u_{k}^{\gamma}\right\rangle\right\rangle .
$$

Similarly

$$
\left\langle\left\langle u_{j}^{\beta}, \sigma_{k}^{* \gamma}\right\rangle\right\rangle=\left\langle\left\langle\sigma_{j}^{\beta}, u_{k}^{\gamma}\right\rangle\right\rangle .
$$

From the definition of $u_{k}^{\gamma}$ (5.22) and the identity (5.7),

$$
\left\langle\left\langle T \nabla_{i} I_{\alpha}, \sigma_{k}^{* \gamma}\right\rangle\right\rangle=\left\langle\left\langle\sum_{\beta \geqq 0, j \geqq 1} a_{i, j}^{\alpha \beta} \sigma_{j}^{\beta}+\mathscr{L} g, u_{k}^{\gamma}\right\rangle\right\rangle=\left\langle\left\langle\nabla_{i} I_{\alpha}, u_{k}^{\gamma}\right\rangle\right\rangle=\chi \delta_{i, k} \delta_{\alpha, \gamma} V\left(\nabla_{i} I_{\alpha}\right) .
$$

Hence we get the result.

Proof of (ii). The diffusion coefficient is a matrix $D_{i, j}^{\alpha, \beta}$ characterized by

$$
\sigma_{i}^{* \alpha}-\sum_{j \geqq 1, \beta \geqq 0} D_{i j}^{\alpha, \beta} \nabla_{j} I_{\beta} \in \overline{\mathscr{L}^{* \mathscr{G}}}
$$

From (a) of (i),

$$
\left\langle\left\langle\left(\sigma_{i}^{* \alpha}-\sum_{j \geqq 1, \beta \geqq 0} D_{i, j}^{\alpha, \beta} \nabla_{j} I_{\alpha}\right), T \nabla_{k} I_{\gamma}\right\rangle\right\rangle=0
$$

for all $k$ and $\gamma$. From (b) of (i),

$$
\sum_{j \geqq 1, \beta \geqq 0} D_{i, j}^{\alpha, \beta} M_{j, k}^{\beta, \gamma}=\chi \delta_{i, k} \delta_{\alpha, \gamma} V\left(\nabla_{i} I_{\alpha}\right)
$$

Hence

$$
D_{i, j}^{\alpha, \beta}=\chi\left(M^{-1}\right)_{i, j}^{\alpha, \beta} V\left(\nabla_{i} I_{\alpha}\right) .
$$

Since $T$ is bounded above by 1 , so is $M$ (as a quadratic form). Hence as a quadratic form we have $D \geqq D_{s}$. The proof of $D \neq D_{s}$ is similar to the one in [8]; $D=D_{s}$ if and only if $\left\langle\left\langle T \nabla_{i} I_{\alpha}, \nabla_{j} I_{\beta}\right\rangle\right\rangle=\delta_{i, j} \delta_{\alpha, \beta} V\left(\nabla_{i} I_{\alpha}\right)$. This implies $T \nabla_{i} I_{\alpha}=\nabla_{i} I_{\alpha}+\mathscr{L}_{s} \phi$. But $\phi=0$ because otherwise $\|T\|>1$. Hence $T \nabla_{i} I_{\alpha}=\nabla_{i} I_{\alpha}$. By (a) of (i) it follows that $\nabla_{j} I_{\alpha}(\eta) \perp \mathscr{L}^{*} \mathscr{G}$ and this leads to contradiction.

Finally, we claim from symmetry arguments,

$$
\left\langle\left\langle\nabla_{i} I_{\alpha}, T \nabla_{j} I_{\beta}\right\rangle\right\rangle=0, \quad \text { if } \delta_{k, i}+\delta_{k, j}+\delta_{k, \alpha}+\delta_{k, \beta}=1 \text { or } 3 \text { for some } 1 \leqq k \leqq d .
$$


Consider the reflection symmetry

$$
\begin{aligned}
R_{1}(x) & =\left(-x_{1}, x_{2}, x_{3}\right), \quad R_{1}(v)=\left(-v_{1}, v_{2}, v_{3}\right), \\
R_{1}(\eta(x, v)) & =\eta\left(R_{1} x, R_{1} v\right), \quad x \in \mathbb{Z}, v \in \mathscr{V} .
\end{aligned}
$$

Clearly, the commutator $\left[R_{1}, \mathscr{L}\right]=0$. Hence $\left[R_{1}, T\right]=0$. Furthermore, $R_{1}$ is an isometry on $\mathscr{G}$ with inner product $\langle\langle\cdot, \cdot\rangle\rangle$. Together with $\left[R_{1}, T\right]=0$, we have

$$
\left\langle\left\langle R_{1} g, T R_{1} h\right\rangle\right\rangle=\langle\langle g, T h\rangle\rangle .
$$

By definition of $R_{1}$, if $i \neq 1$, then $R_{1}\left(\nabla_{i} I_{1}\right)=-\nabla_{i} I_{1}$. Similarly, if $\alpha \neq 1$, then $R_{1}\left(\nabla_{1} I_{\alpha}\right)=-\nabla_{1} I_{\alpha}$. On the other hand, if $\alpha \neq 1$ and $i \neq 1$ or if $\alpha=i=1$, then $R_{1}\left(\nabla_{i} I_{\alpha}\right)=\nabla_{i} I_{\alpha}$. Hence if the indices satisfy the condition of (5.28) for $k=1$ then

$$
\left\langle\left\langle\nabla_{i} I_{\alpha}, T \nabla_{j} I_{\beta}\right\rangle\right\rangle=\left\langle\left\langle R_{1}\left(\nabla_{i} I_{\alpha}\right), R_{1}\left(T \nabla_{j} I_{\beta}\right)\right\rangle\right\rangle=-\left\langle\left\langle\nabla_{i} I_{\alpha}, T \nabla_{j} I_{\beta}\right\rangle\right\rangle
$$

Hence $\left\langle\left\langle\nabla_{i} I_{\alpha}, T \nabla_{j} I_{\beta}\right\rangle\right\rangle=0$. This proves (5.28).

Furthermore, there are permutation symmetries. Let $R_{k, \ell}(k \neq \ell)$ denote the permutation of the coordinate axis $k \rightarrow \ell$. Then

$$
\left\langle\left\langle\nabla_{i} I_{\alpha}, T \nabla_{j} I_{\beta}\right\rangle\right\rangle=\left\langle\left\langle\nabla_{R_{k, \ell} i} I_{R_{k, \ell} \alpha}, T \nabla_{R_{k, \ell} j} I_{R_{k, \ell} \beta}\right\rangle\right\rangle \quad\left(R_{k \ell} \alpha=\alpha \text { if } \alpha=0\right) \text {. }
$$

Since $D$ inherits the symmetries of $\left\langle\left\langle\nabla_{i} I_{\alpha}, T \nabla_{j} I_{\beta}\right\rangle\right\rangle$, (5.25) holds. This proves (ii). Proof of (iii). Suppose $\xi=\sum_{i \geqq 1, \beta \geqq 0} b_{i}^{\beta} u_{i}^{\beta}+\mathscr{L}_{s} g$. From (5.23),

$$
\left\langle\left\langle\xi, T^{-1} \xi\right\rangle\right\rangle=\left\langle\left\langle\sum_{i \geqq 1, \beta \geqq 0} b_{i}^{\beta} u_{i}^{\beta}+\mathscr{L}_{s} g, \sum_{i \geqq 1, \beta \geqq 0} b_{i}^{\beta} \sigma_{i}^{\beta}+\mathscr{L} g\right\rangle\right\rangle .
$$

Hence (iii) follows from explicit computation using Lemmas 5.2, 5.4 and the definition of $u_{i}^{\beta}$.

Remark. The inverse of $T, R$, was considered in [8]. The proof of its boundedness given there, based on open mapping principle, is incorrect. But the operator $R$ was only used to derive a variational formula for the diffusion coefficient. The variational formula in [8] is indeed correct and only minor modifications are needed to prove it.

Starting from [26] one can get variational characterizations for the diffusion coefficient and for its inverse. These formulas have been given in [26] for the asymmetric simple exclusion process.

Theorem 5.8. The following variational representation for the diffusion matrix $D$ holds: For any $a=\left\{a_{j}^{\alpha} \in \mathbb{R}, \alpha=0, \ldots, d, j=1, \ldots, d\right\}$,

$$
\begin{gathered}
a \cdot D a=\frac{1}{\Theta} \inf _{h \in \mathscr{G}}\langle\langle\sigma \cdot a+\mathscr{L} h, \sigma \cdot a+\mathscr{L} h\rangle\rangle, \\
a \cdot D^{-1} a=\frac{1}{\Theta} \inf _{h \in \mathscr{G}}\langle\langle\nabla I \cdot a+\mathscr{L} h, \nabla I \cdot a+\mathscr{L} h\rangle\rangle .
\end{gathered}
$$


Here $\Theta=\chi V\left(\nabla_{i} I_{\alpha}\right)$ is the "susceptibility." Moreover, we used the notation

$$
\begin{gathered}
\nabla I=\left\{\nabla_{i} I_{\alpha}, \alpha=0, \ldots, d, i=1, \ldots, d\right\}, \quad a \cdot v=\sum_{\gamma=0}^{d} \sum_{k=1}^{d} a_{k}^{\gamma} b_{k}^{\gamma}, \\
a \cdot A a=\sum_{\alpha, \gamma=0}^{d} \sum_{j, k=1}^{d} A_{j, k}^{\alpha, \gamma} a_{j}^{\alpha} a_{k}^{\gamma} .
\end{gathered}
$$

Proof. The proof follows closely the arguments in [26], taking advantage of the fact that $M$ and $D$ are symmetric because of the isotropy. We report it for sake of completeness. First note that, because of the isotropy, $V\left(\nabla_{j} I_{\alpha}\right)$ is independent on $i, \alpha$.

From part (a) of the statement (i) in Theorem $5.7 \mathscr{L}^{*} \mathscr{G} \perp T \mathscr{G}^{0}$, where

$$
T \mathscr{G}^{0}=\left\{\sum_{\alpha=0}^{d} \sum_{j=1}^{d} a_{j}^{\alpha} T \nabla_{j} I_{\alpha}\left(\eta_{0}\right), a_{j}^{\alpha} \in \mathbb{R}\right\} .
$$

Note that the dimension of $\mathscr{G}^{0}$ and $\mathscr{G}_{w}$, defined in (ii) of Theorem 5.6, are the same. From part (b) of (i) in Theorem 5.7 and (ii) of Theorem 5.6, we have the following orthogonal decomposition of $\overline{\mathscr{G}}$ :

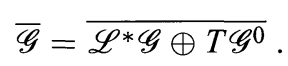

Hence the current can be represented, up to some approximation, as

$$
\sigma^{*}=A(T \nabla I)+\mathscr{L}^{*} g,
$$

for a suitable $g \in \mathscr{G}$ and a suitable matrix $A$. It is easy to check that $A$ can be identified with $D^{-1}$, by multiplying previous relation by $T \nabla I$ and using (ii) of Theorem 5.7. As a consequence, we have

$$
\inf _{g \in \mathscr{G}} V\left(a \cdot\left[\sigma^{*}-D(T \nabla I)\right]-\mathscr{L}^{*} g\right)=0
$$

From (iii) of Theorem 5.7,

$$
\langle\langle\xi, T \xi\rangle\rangle=\left\langle\left\langle T^{*} \xi,\left(T^{*}\right)^{-1} T^{*} \xi\right\rangle\right\rangle=\left\langle\left\langle T^{*} \xi, T^{*} \xi\right\rangle\right\rangle .
$$

Hence, taking into account (i) and (ii) in Theorem 5.7, one gets (5.30).

To prove the formula for $D^{-1}$ we recall that by definition $D$ is such that, up to some approximation,

$$
\sigma^{*}-D \nabla I-\mathscr{L}^{*} g=0
$$

for some $g$. Substituting this in (5.30) we get

$$
a \cdot D a=\inf _{g \in \mathscr{G}} \frac{1}{\bar{\chi}} V\left(a \cdot D \nabla I-\mathscr{L}^{*} g\right) .
$$

Put $b=D a$. The previous formula becomes (5.31). This concludes Theorem 5.8. 


\section{Remarks.}

1) The variational formulas easily provide upper and lower bounds for $D$ (see [26]).

2) We can combine Theorems 5.8 and 5.3 to obtain variational formulas involving only the scalar product $\langle\cdot, \cdot\rangle_{0}$ and expectations w.r.t. the equilibrium measure. Since this formula is quite complicated and its derivation from Theorems 5.8 and 5.3 is straightforward, we shall not present it here. A similar formula for the simple exclusion process has been given in details in [8].

3 ) Note that $D_{1}$ and $D_{2}$ in (5.25) contribute to the shear viscosity, while the bulk viscosity is $\zeta=D_{3}+D_{4}$. The coefficient $D_{0}$ does not play any role in the present setup. In fact it would be relevant only in the behavior of the total density $I_{0}$, which differs from a constant for terms of higher order in $\varepsilon$ than $I_{\alpha}$ for $\alpha>0$, because of the incompressible limit. Therefore we do not need to discuss its evolution.

To conclude this section we note that it is possible to connect the diffusion matrix we found to a Green-Kubo-type expression. The connection is only formal because it is hard to give a rigorous meaning to such an expression. The formal argument follows the one presented for the simple exclusion process in [27].

By the definition of $D, \sigma-D \nabla I \in \mathscr{L} \mathscr{G}$. Therefore, there are local functions $g=\left\{g_{j}^{\alpha}, \alpha=0, \ldots, d, j=1, \ldots, d\right\}$ such that,

$$
\left\|\sigma_{j}^{\alpha}-\sum_{\gamma, k} D_{j, k}^{\alpha, \gamma} \nabla_{k} I_{\gamma}-\mathscr{L} g_{j}^{\alpha}\right\|_{-1}=0 .
$$

Of course, such a relation is not literally true, but some approximation arguments are necessary.

We claim that for any $a=\left\{a_{j}^{\alpha} \in \mathbb{R}, \alpha=0, \ldots, d, j=1, \ldots, d\right\}$,

$$
a \cdot D a=\chi a \cdot a+\chi \Theta^{-1}\left\langle\left\langle\mathscr{L} g \cdot a, \mathscr{L}_{s} g \cdot a\right\rangle\right\rangle .
$$

In fact, from part (ii) of Theorem 5.7,

$$
a \cdot(\Theta D)^{-1} a=\langle\langle a \cdot \nabla I, T a \cdot \nabla I\rangle\rangle .
$$

Replacing $\nabla I$ with $D^{-1}[\sigma-\mathscr{L} g]$ and recalling that $T(\sigma+\mathscr{L} g)=\chi \nabla I+\mathscr{L}_{s} G$, we have

$$
\begin{aligned}
\chi^{-1} \Theta a \cdot D a= & \left\langle\left\langle a \cdot(\sigma-\mathscr{L} g), a \cdot\left(\nabla I-\mathscr{L}_{s} g\right)\right\rangle\right\rangle \\
= & \langle\langle a \cdot \sigma, a \cdot \nabla I\rangle\rangle+\left\langle\left\langle\mathscr{L} g \cdot a, \mathscr{L}_{s} g \cdot a\right\rangle\right\rangle \\
& +\langle\langle\mathscr{L} g \cdot a, \nabla I \cdot a\rangle\rangle+\left\langle\left\langle\sigma \cdot a, \mathscr{L}_{s} g \cdot a\right\rangle\right\rangle .
\end{aligned}
$$

By Lemma 5.4 the first term is just $\Theta a \cdot a$.

Now we use the fact that $\nabla I$ is equivalent to 0 in the scalar product $\langle\cdot, \cdot\rangle_{0}$. We have

$$
\langle\langle\mathscr{L} g \cdot a, \nabla I \cdot a\rangle\rangle+\left\langle\left\langle\sigma \cdot a, \mathscr{L}_{s} g \cdot a\right\rangle\right\rangle=-\left\langle\sigma^{*} \cdot a, g \cdot a\right\rangle_{0}-\langle\sigma \cdot a, g \cdot a\rangle_{0}=0,
$$

because $\sigma+\sigma^{*}=2 \chi \nabla I$. This proves (5.32). 
By Lemma 5.2, and the relation $\mathscr{L} g=[\sigma-D \nabla I]$, we have

$$
\begin{aligned}
\left\langle\left\langle\mathscr{L} g \cdot a, \mathscr{L}_{s} g \cdot a\right\rangle\right\rangle & =-\langle\mathscr{L} g \cdot a, g \cdot a\rangle_{0} \\
& =-\left\langle[\sigma-D \nabla I] \cdot a \mathscr{L}^{-1}[\sigma-D \nabla I] \cdot a\right\rangle_{0}=-\left\langle\sigma \cdot a \mathscr{L}^{-1} \sigma \cdot a\right\rangle_{0} .
\end{aligned}
$$

Since $-\sigma=\sigma^{*}-2 \chi \nabla I$, we have

$$
a \cdot D a=\chi a \cdot a-\frac{\chi}{\Theta}\left\langle\sigma^{*} \cdot a \mathscr{L}^{-1} \sigma \cdot a\right\rangle_{0} .
$$

The formal relation $(-\mathscr{L})^{-1}=\int_{0}^{\infty} d t \exp [\mathscr{L} t]$ implies the Green-Kubo formula for this model:

$$
D_{i, j}^{\alpha, \beta}=\chi \delta_{i \cdot j} \delta_{\alpha, \beta}-\frac{\chi}{\Theta} \int_{0}^{\infty} d t\left\langle\sigma_{i}^{* \alpha} \exp [\mathscr{L} t] \sigma_{j}^{\beta}\right\rangle_{0}
$$

Here

$$
\left\langle\sigma_{i}^{* \alpha} \exp [\mathscr{L} t] \sigma_{j}^{\beta}\right\rangle_{0}=\sum_{x} E^{\mu_{r}}\left[\sigma_{i}^{* \alpha}(t) \tau_{x} \sigma_{j}^{\beta}(0)\right]
$$

is the integrated current-current time self correlation.

In the Green-Kubo formula (5.33) the first term represents the contribution to the diffusion due to the symmetric simple exclusion process, while the second one is the deterministic diffusion, which is positive by part (ii) of Theorem 5.7, provided that the integral is finite.

\section{Eigenvalues Estimates}

In this section we prove Theorem 3.10, which is parallel to Theorem 4.2 in [8] for the symmetric simple exclusion process. If one can establish the spectral gap and the log-Sobolev inequality for the full dynamics $\mathscr{L}_{s}+\mathscr{L}^{c}$, then a parallel proof will conclude Theorem 3.10. We do not follow this route because the spectral gap and log-Sobolev for $\mathscr{L}_{s}^{e x}+\mathscr{L}^{c}$ are hard to obtain. Instead, we shall control the fast modes $I^{-}$, which are conserved quantities for $\mathscr{L}_{s}^{e x}$ but not for $\mathscr{L}^{c}$, using the following lemma.

Lemma 6.1. Put $Q f \equiv \sum_{q \in 2} Q_{0}^{q} f$. Suppose that $I_{\alpha}, \quad \alpha=-N+d+1, \ldots,-1$, defined as in (4.1), satisfy

$$
E^{\mu}\left[I_{\alpha}\left(\eta_{x}\right) ; I_{\beta}\left(\eta_{x}\right)\right]=0, \quad \alpha<0, \beta<0, \alpha \neq \beta,
$$

where $\mu$ is any equilibrium measure for the whole dynamics, i.e.

$$
\mu=Z_{r, n}^{-1} \exp \left\{\sum_{\alpha=0}^{d} n_{\alpha} I_{\alpha}(\eta)\right\} \mu_{r} .
$$

Let $z_{\alpha}=E^{\mu}\left[I_{\alpha}\left(\eta_{x}\right)\right]$. Then there exist a local function $v$ and a function $g \in \mathscr{G}_{z}^{\text {ex }}$ such that

$$
I_{\alpha}\left(\eta_{x}\right)-z_{\alpha}=(Q v)\left(\eta_{x}\right)+g\left(\eta_{x}\right), \quad \alpha<0 .
$$

Here $\mathscr{G}_{z}^{e x}$ is the analog of $\mathscr{G}^{e x}$ with the measure $\mu_{r}$ replaced by $\mu$ given by (6.2) with chemical potentials $n_{\alpha}$ chosen so that $E^{\mu}\left[I_{\alpha}\left(\eta_{x}\right)\right]=z_{\alpha}, \alpha=0, \ldots, d$. 
Lemma 6.1 is exactly the same as Lemma 4.5 except that the measure is not restricted to $\mu_{r}$. Note the orthogonality relation (6.1) is the same as (4.3) except that the reference measure was changed accordingly. The proof of Lemma 6.1 is exactly the same as Lemma 4.5 and we omit it.

Recall the definitions of $\hat{\omega}, \omega$ and $\tilde{\Lambda}_{k}$ in (3.A). Denote $x \in \Lambda_{\bar{\ell}_{1}, \sigma}$ by $x \in \sigma$, and let $\bar{I}_{\sigma}^{+}=\bar{I}_{\Lambda_{i, \sigma}}^{+}$For any local function $h_{x}$, let

$$
\bar{h}_{\sigma}=E^{\mu_{r}}\left[\operatorname{Av}_{x \in \sigma} h_{x} \mid \bar{I}_{\sigma}^{+}\right]
$$

From the definition of $\hat{\omega}$ we have $(\hat{\omega} * h)(x)=\mathrm{Av}_{|\sigma-x| \leqq k} \bar{h}_{\sigma}$, where $\sigma \in(2 \bar{\ell}+1) \mathbb{Z}^{d}$. For the rest of this chapter, $A v_{|\sigma-x| \leqq k}$ takes this meaning and we shall not repeat it. The following theorem is a restatement of Theorem 4.6 in [8]. Indeed, Theorem 6.2 summarizes the result for the "case 1" in the proof of Theorem 4.6 in [8]. The proof given in [8] is somehow sketchy; details can be found in [25].

Theorem 6.2. Suppose $h$ is a local function. Then for any constant $\gamma>0$ and smooth function $J$,

$$
\begin{gathered}
\lim _{\ell \rightarrow \infty} \lim _{\varepsilon \rightarrow 0}\left\{\varepsilon^{d-2} \int f \sum_{x \in \Lambda_{L}} J(\varepsilon x)\left[\underset{|\sigma-x|<k}{\operatorname{Av}} \bar{h}_{\sigma}-E^{\mu_{r}}\left[\underset{|\sigma-x|<k}{\operatorname{Av}} \bar{h}_{\sigma} \mid \bar{I}_{k, x}\right]\right] d \mu_{r}\right. \\
\left.-\gamma \varepsilon^{d-4} D_{\Lambda_{L}}^{e x}(\sqrt{f})\right\} \leqq 0 .
\end{gathered}
$$

Here $k=\ell \varepsilon^{-2 / d}$ and $E^{\mu_{r}}\left[h \mid \bar{I}_{k, x}\right]$ has been defined in Sect. $4, \bar{I}_{k}=\left\{\bar{I}_{\alpha, k}, \alpha=-N+\right.$ $d+1, \ldots, d\}$. Finally $D^{\text {ex }}$ is the Dirichlet form associated to $\mathscr{L}_{s}^{\text {ex }}$, defined in (3.14).

Proof of Theorem 3.10. Step 1. Consider the decomposition

$$
\operatorname{Av}_{x \in \sigma} h_{x}=\left(\operatorname{Av}_{x \in \sigma} h_{x}-\bar{h}_{\sigma}\right)+\bar{h}_{\sigma}:=\tilde{h}_{\sigma}+\bar{h}_{\sigma}
$$

Since $E^{\mu_{r}}\left[\tilde{h}_{\sigma} \mid \bar{I}_{\sigma}^{+}\right]=0$, we can bound $\tilde{h}_{\sigma}$ with standard perturbation argument so that

$$
\begin{gathered}
\lim _{\ell \rightarrow \infty} \lim _{\varepsilon \rightarrow 0}\left\{\varepsilon^{-2} \int f \operatorname{Av}_{x} J(\varepsilon x)\left[\underset{|\sigma-x|<k}{\operatorname{Av}} \tilde{h}_{\sigma}\right] d \mu_{r}-\text { const. } \gamma^{-1} \varepsilon^{d} \sum_{x \in \Lambda_{L}} J^{2}(\varepsilon x)\right. \\
\quad \times \underset{|\sigma-x| \leqq k}{\left.\operatorname{Av}_{1} \int V_{\ell}\left(h, \bar{I}_{\sigma}^{+}\right) f d \mu_{r}-\gamma \varepsilon^{d-4} D_{\Lambda}(\sqrt{f})\right\} \leqq 0}
\end{gathered}
$$

From the entropy bound and the fact that $\psi$ is a local Gibbs state defined in (3.1), one can check that

$$
\begin{aligned}
& \lim _{\ell \rightarrow \infty} \lim _{\varepsilon \rightarrow 0}\left\{\varepsilon^{d} \sum_{x \in \Lambda_{L}} \underset{|\sigma-x| \leqq k}{\operatorname{Av}} J(\varepsilon x)^{2} \int V_{\ell}\left(h, \bar{I}_{\sigma}^{+}\right) f d \mu_{r}-\varepsilon^{-1} s(f \mid \psi)\right\} \\
& \leqq \text { const. } \int J(z)^{2} d z V(h, r) .
\end{aligned}
$$


Hence the second term converges to the second term in (3.29). This procedure was carried out in details in e.g., $[16,8]$ and we omit the details (see Sect. 6 of [8], pp. 1264-1266).

Step 2. We now bound $\bar{h}_{\sigma}$. Applying Theorem 6.2, one has

$$
\begin{gathered}
\lim _{\ell \rightarrow \infty} \lim _{\varepsilon \rightarrow 0}\left\{\varepsilon^{d-2} \int f \sum_{x \in \Lambda_{L}} J(\varepsilon x)\left[\underset{|\sigma-x|<k}{\operatorname{Av}} \bar{h}_{\sigma}-E^{\mu_{r}}\left[\underset{|\sigma-x|<k}{\operatorname{Av}} \bar{h}_{\sigma} \mid \bar{I}_{k, x}\right]\right] d \mu_{\beta}\right. \\
\left.-\gamma \varepsilon^{d-4} D_{\Lambda_{L}}(\sqrt{f})\right\} \leqq 0 .
\end{gathered}
$$

Note that the conditional expectation is w.r.t. $\bar{I}_{k}$ on the block $\Lambda_{k}$ and $\bar{I}_{k}=\left\{\bar{I}_{\alpha, k}\right.$, $\alpha=-N+d+1, \ldots, d\}$. We would like to replace the canonical expectation by the grand canonical expectation. More precisely, let $\mu_{2, w}$ be the grand canonical measure such that

$$
E^{\mu_{2, w}}\left[I_{\alpha}\right]=w_{\alpha}, \quad \alpha=-N+d+1, \ldots, d ;
$$

explicitly $\mu_{2, w}$ is the product measure with marginal measure given by

$$
\mu_{2, w}=Z_{r, n}^{-1} \exp \left\{\sum_{\alpha=-N+d+1}^{d} \lambda_{\alpha} I_{\alpha}(\eta)\right\} \mu_{r}
$$

Note that in the definition of $\mu_{2, w}$ we specify the averages of $I_{\alpha}$ also for $\alpha>0$, while in the definition of $\mu_{1, w}$ in Sect. 3 we only specified the averages of $I_{\alpha}$ for $\alpha<0$. Fixed the local empirical averages $\bar{I}_{\alpha, k}$ the corresponding grand canonical expectation is defined by

$$
E^{\mu_{2, w}}\left[\underset{|\sigma-x|<k}{\operatorname{Av}} \bar{h}_{\sigma}\right]
$$

with $w=\bar{I}_{k, x}$. Let $\vartheta_{x}$ denote the difference

$$
\vartheta_{x}=E^{\mu_{2, w}}\left[\underset{|\sigma-x|<k}{\operatorname{Av}} \bar{h}_{\sigma}\right]-E^{\mu_{r}}\left[\underset{|\sigma-x|<k}{\operatorname{Av}} \bar{h}_{\sigma} \mid \bar{I}_{k, x}\right] .
$$

We claim that this difference is negligible in the sense that for any $\gamma>0$,

$$
\lim _{\ell \rightarrow \infty} \lim _{\varepsilon \rightarrow 0}\left\{\varepsilon^{d-2} \int f \sum_{x \in \Lambda_{L}} J(\varepsilon x) \vartheta_{x} d \mu_{r}-\gamma \varepsilon^{-2} s(f)\right\} \leqq 0 .
$$

The strong equivalence of ensembles states that $\vartheta_{x}$ is small provided $\bar{I}_{k, x}$ is bounded away from 0 or 1 . A precise statement is given in Corollary 6.3 in [22]. We state a simplified version in our context: For some small constant $\gamma$,

$$
\left|\vartheta_{x}\right| \leqq \begin{cases}k^{-d} & \text { if } k^{-\gamma} \leqq \bar{I}_{k, x} \leqq 1-k^{-\gamma} \\ k^{-d / 2} & \text { otherwise }\end{cases}
$$


The second bound is proved in [22] only if $\bar{I}_{k, x}$ is bounded away from 0 or 1 in the sense that $k^{-d / 2} \leqq \bar{I}_{k, x} \leqq 1-k^{-d / 2}$. On the other hand, if $\bar{I}_{k, x} \leqq k^{-d / 2}$, then the number of particles for each color is less than $k^{-d / 2}$. Hence both $E^{\mu_{2, w}}\left[\mathrm{Av}_{|\sigma-x|<k} \bar{h}_{\sigma}\right]$ and $E^{\mu_{r}}\left[\operatorname{Av}_{|\sigma-x|<k} \bar{h}_{\sigma} \mid \bar{I}_{k, x}\right]$ are bounded by $C k^{-d / 2}$ and (6.8) holds trivially.

From (6.8) one has

$$
\left|\varepsilon^{d} E^{\mu_{r}}\left[\sum_{x \in \Lambda_{L}} J(\varepsilon x) \vartheta_{x}\right]\right| \leqq C k^{-d} .
$$

From the entropy inequality the left side of (6.7) is bounded by

$$
\varepsilon^{d-2} \log E^{\mu_{r}}\left[\exp \left\{\sum_{x \in \Lambda_{L}} J(\varepsilon x) \vartheta_{x}\right\}\right] .
$$

It is now standard to bound the last term by

$$
\begin{aligned}
& \varepsilon^{d-2} \log E^{\mu_{r}}\left[\exp \left\{\sum_{x \in \Lambda_{L}} J(\varepsilon x) \vartheta_{x}\right\}\right] \\
& \leqq \varepsilon^{d-2} E^{\mu_{r}}\left[\sum_{x \in \Lambda_{L}} J(\varepsilon x) \vartheta_{x}\right]+C \varepsilon^{d-2} \sum_{x \in \Lambda_{L}} E^{\mu_{r}}\left[\vartheta_{x}^{2}\right]+C \varepsilon^{-2} k^{-d} \leqq C \varepsilon^{-2} k^{-d} .
\end{aligned}
$$

Taking the limit and remembering $k=\ell \varepsilon^{-2 / d}$, we have proved

$$
\begin{gathered}
\lim _{\ell \rightarrow \infty} \lim _{\varepsilon \rightarrow 0}\left\{\varepsilon^{d-2} \int f \sum_{x \in \Lambda_{L}} J(\varepsilon x)\left[\underset{|\sigma-x|<k}{\operatorname{Av}} \bar{h}_{\sigma}-E^{\mu_{1} \bar{I}_{k, x}}\left[\underset{|\sigma-x|<k}{\operatorname{Av}} \bar{h}_{\sigma}\right]\right] d \mu_{r}\right. \\
\left.-\gamma \varepsilon^{d-4} D_{\Lambda_{L}}(\sqrt{f})\right\}-\delta^{-1} \varepsilon^{-2} s(f \mid \psi) \leqq 0 .
\end{gathered}
$$

The change from $s(f)$ to $s(f \mid \psi)$ is not important in the previous argument.

Step 3. We would like to replace the expectation $E^{\mu_{2, w}}$ with the one w.r.t. $\mu_{1, Y}$ defined in Sect. 3, with $y_{\alpha}=w_{\alpha}$ for $\alpha=0, \ldots, d$. Let us consider the difference

$$
u(w)=E^{\mu_{2, w}}\left[\underset{|\sigma-x|<k}{\operatorname{Av}} \bar{h}_{\sigma}\right]-E^{\mu_{1, Y}}\left[\underset{|\sigma-x|<k}{\operatorname{Av}} \bar{h}_{\sigma}\right] .
$$

To prove Theorem 3.10, it suffices to prove, for $\delta$ small enough,

$$
\lim _{\ell \rightarrow \infty} \lim _{\varepsilon \rightarrow 0}\left\{\varepsilon^{d-2} \int f \sum_{x \in \Lambda_{L}} J(\varepsilon x) \tau_{x} u d \mu_{r}-\gamma \varepsilon^{d-4} D_{\Lambda_{L}}(\sqrt{f})-\delta^{-1} \varepsilon^{-2} s(f \mid \psi)\right\} \leqq 0 .
$$

Step 4. Since $\mu_{i}$ are product measures for $i=1,2$, we can drop the average over $\sigma$ and $u$ is simply

$$
u(w)=E^{\mu_{2, w}}\left[E^{\mu_{r}}\left[h \mid \bar{I}_{\bar{\ell}}^{+}\right]\right]-E^{\mu_{1, Y}}\left[E^{\mu_{r}}\left[h \mid \bar{I}_{\bar{\ell}}^{+}\right]\right] .
$$


Note that from the definition of $\mu_{i}, i=1,2$, we have

$$
E^{\mu_{2, w}}\left[I_{\alpha}\right]=w_{\alpha}=Y_{\alpha}=E^{\mu_{1, Y}}\left[I_{\alpha}\right], \quad \alpha=0, \ldots, d .
$$

But this equality fails for $\alpha<0$. Furthermore, even though (6.12) holds, it fails if $I_{\alpha}$ is replaced by nonlinear functionals of $I_{\alpha}(\alpha \geqq 0)$.

Define

$$
\hat{u}_{x}(w)=\tau_{x} u(w)-\phi_{x}=\tau_{x} u(w)-\sum_{\alpha=-N+(d+1)}^{d} c_{\ell, \alpha, x}\left(I_{\alpha, x}-z_{\alpha, x}\right),
$$

where

$$
z_{\alpha, x}=E^{\psi}\left[I_{\alpha, x}\right], \quad c_{\ell, \alpha, x}=\left.\frac{\partial u}{\partial w_{\alpha}}\right|_{w=z_{x}} .
$$

From the entropy inequality one has for $\delta>0$,

$$
\begin{aligned}
& \varepsilon^{-2} \int f \underset{x \in \Lambda_{L}}{\operatorname{Av}} J(\varepsilon x) \hat{u}_{x} d \mu \\
& \quad \leqq \delta^{-1} \varepsilon^{-2+d} \log E^{\psi}\left[\exp \left\{\delta \sum_{x} J(\varepsilon x) \hat{u}_{x}\right\}\right]+\delta^{-1} \varepsilon^{-2} s(f \mid \psi)
\end{aligned}
$$

By definition of $c_{\ell, \alpha, x}$, we can apply Lemma 3.7 to bound the first term on the right-hand side of (6.14) provided $\delta$ is small enough. Hence one has

$$
\lim _{\ell \rightarrow \infty} \lim _{\varepsilon \rightarrow 0} \delta^{-1} \varepsilon^{-2+d} \log E^{\psi}\left[\exp \left\{\delta \sum_{x \in \Lambda_{L}} J(\varepsilon x) \hat{u}_{x}\right\}\right]=0
$$

provided $\delta$ is small enough. Therefore, to prove Theorem 3.10 , we only have to bound $\phi_{x}$ defined by the second of (6.13), in the sense that for any $\gamma>0$ and $\delta$ sufficiently small,

$$
\lim _{\ell \rightarrow \infty} \lim _{\varepsilon \rightarrow 0}\left\{\varepsilon^{d-2} \int f \sum_{x \in \Lambda_{L}} J(\varepsilon x) \phi_{x} d \mu_{r}-\gamma \varepsilon^{d-4} D_{\Lambda_{L}}(\sqrt{f})-\delta^{-1} \varepsilon^{-2} s(f \mid \psi)\right\} \leqq 0
$$

Step 5. We claim the following estimates hold:

$$
c_{\alpha, \ell, x}=0, \quad \alpha \geqq 0,
$$

and

$$
\lim _{\ell \rightarrow \infty} c_{\alpha, \ell, x}=0, \quad \alpha<0 .
$$

We shall prove these two estimates in Step 6. Assuming these two estimates, we now prove (6.16).

From Lemma 6.1, one has, for all $\alpha<0, I_{\alpha, x}-z_{\alpha, x}=Q_{x} v_{x}+\xi_{\alpha, x}$ with $\xi_{\alpha, x} \in$ $\tilde{\mathscr{G}}_{z_{x}}^{e x}$. From the integration by parts,

$$
\int f \sum_{x \in \Lambda_{L}} Q_{x} v_{x} d \mu \leqq \text { const. } D_{\Lambda_{L}}(\sqrt{f})
$$


Hence (6.16) holds if we neglect the contribution of $\xi_{\alpha, x} \in \tilde{\mathscr{G}}_{z_{x}}^{e x} x$. On the other hand, from the entropy bound,

$$
\begin{aligned}
& \varepsilon^{-2} \int f \underset{x \in \Lambda_{L}}{\operatorname{Av}} J(\varepsilon x) \xi_{\alpha, x} d \mu \\
& \quad \leqq \delta^{-1} \varepsilon^{-2+d} \log E^{\psi}\left[\exp \left\{\delta \sum_{x \in \Lambda_{L}} J(\varepsilon x) \xi_{\alpha, x}\right\}\right]+\delta^{-1} \varepsilon^{-2} s(f \mid \psi) .
\end{aligned}
$$

Because $\xi_{\alpha, x} \in \tilde{\mathscr{G}}_{z_{x}}^{e x}$, we can apply Lemma 3.7 and this concludes the proof of (6.16). Step 6. Finally we have to prove (6.17) and (6.18). From (6.12), the value of $u(w)$ does not change if we replace $h$ in the definition (6.11) by

$$
h_{x}:=h-\zeta_{x}:=h-\sum_{\alpha \geqq 0}\left(e+\varepsilon a_{\alpha, x}\right)\left(I_{\alpha, x}-z_{\alpha, x}\right)
$$

for some constants $e$ and $a_{\alpha, x}$.

Since $\psi$ is a local Gibbs state given by (3.1), we can choose constants $e$ and $a_{\alpha, x}$ such that $h_{x} \in \tilde{\mathscr{G}}_{z_{x}}$. Because the statements of (6.17) and (6.18) are local, we shall drop the subscript $x$ and assume that, without loss of generality, $h_{x} \in \tilde{\mathscr{G}}_{z_{\mathrm{r}}}$.

By definition one has the identity

$$
\begin{aligned}
\left.\frac{\partial u}{\partial w_{\alpha}}\right|_{w=z}= & \sum_{\beta=-N+d+1}^{d} \sum_{x}\left\{E^{\mu_{2, w}}\left[E^{\mu_{r}}\left[h \mid \bar{I}_{\ell}^{+}\right] ; \tau_{x} I_{\beta}\right] \frac{\partial \lambda_{\beta}}{\partial w_{\alpha}}\right. \\
& \left.-E^{\mu_{1, Y}}\left[E^{\mu_{r}}\left[h \mid \bar{I}_{\ell}^{+}\right] ; \tau_{x} I_{\beta}\right] \frac{\partial n_{\beta}}{\partial w_{\alpha}}\right\}\left.\right|_{w=z} .
\end{aligned}
$$

By definition, $\mu_{2, z}=\mu_{1, z}:=\mu_{z}$ and

$$
\left.\frac{\partial n_{\beta}}{\partial w_{\alpha}}\right|_{w=z}=0, \quad \alpha<0
$$

From the orthogonality relation $E^{\mu_{z}}\left[I_{\alpha} ; I_{\beta}\right]=0$ for $\alpha \neq \beta$, one has

$$
\left.\frac{\partial w_{\alpha}}{\partial \lambda_{\beta}}\right|_{w=z}=0, \quad \text { if } \alpha \neq \beta
$$

Hence

$$
\left.\frac{\partial \lambda_{\beta}}{\partial w_{\alpha}}\right|_{w=z}=0, \quad \text { if } \alpha \neq \beta .
$$

Since $h \in \tilde{\mathscr{G}}_{z}$, we have $\sum_{x} E^{\mu_{z}}\left[E^{\mu_{z}}\left[h \mid \bar{I}_{\ell}^{+}\right] ; \tau_{x} I_{\beta}\right]=0$ if $\beta \geqq 0$. Using these identities in (6.19), we have proved (6.17).

For $\alpha<0$ we have

$$
\left.\frac{\partial u}{\partial w_{\alpha}}\right|_{w=z}=\left.\sum_{x} E^{\mu_{z}}\left[E^{\mu_{z}}\left[h \mid \bar{I}_{\ell}^{+}\right] ; I_{\alpha}\left(\eta_{x}\right)\right] \frac{\partial \lambda_{\alpha}}{\partial w_{\alpha}}\right|_{w=z} .
$$


Using the Schwartz inequality we get

$$
\begin{aligned}
& \sum_{x} E^{\mu_{z}}\left[E^{\mu_{z}}\left[h \mid \bar{I}_{\ell}^{+}\right] ; I_{\alpha}\left(\eta_{x}\right)\right]=E^{\mu_{z}}\left[E^{\mu_{z}}\left[h \mid \bar{I}_{\ell}^{+}\right] ; \sum_{|x| \leqq \ell} I^{-}\left(\eta_{x}\right)\right] \\
& \leqq E^{\mu_{z}}\left[E^{\mu_{z}}\left[h \mid \bar{I}_{\ell}^{+}\right] ; E^{\mu_{z}}\left[h \mid \bar{I}_{\ell}^{+}\right]\right]^{1 / 2} E^{\mu_{z}}\left[\sum_{|x| \leqq \ell} I^{-}\left(\eta_{x}\right) ; \sum_{|x| \leqq \ell} I^{-}\left(\eta_{x}\right)\right]^{1 / 2} \\
& \leqq \ell^{d / 2} E^{\mu_{z}}\left[E^{\mu_{z}}\left[h \mid \bar{I}_{\ell}^{+}\right] ; E^{\mu_{z}}\left[h \mid \bar{I}_{\ell}^{+}\right]\right]^{1 / 2} .
\end{aligned}
$$

Next lemma shows that, since $h \in \tilde{\mathscr{G}}_{z}$, the last term approaches to 0 as $\ell \rightarrow \infty$. This concludes (6.18).

Lemma 6.3. Suppose $h \in \tilde{\mathscr{G}}_{z}$. Then

$$
\lim _{\ell \rightarrow \infty} \ell^{d} E^{\mu_{z}}\left[E^{\mu_{z}}\left[h \mid \bar{I}_{\ell}^{+}\right] ; E^{\mu_{z}}\left[h \mid \bar{I}_{\ell}^{+}\right]\right]=0 .
$$

Proof. Let $\hat{h}(Y)=E^{\mu_{1, Y}}[h]$, where $\mu_{1, Y}$ was defined in (3.16). It is not hard to check that the equivalence of ensembles implies that for some $\gamma>0$,

$$
E^{\mu_{z}}\left[\left(E^{\mu_{z}}\left[h \mid \bar{I}_{\ell}^{+}\right]-\hat{h}\left(Y=\bar{I}_{\ell}^{+}\right)\right) ;\left(E^{\mu_{z}}\left[h \mid \bar{I}_{\ell}^{+}\right]-\hat{h}\left(Y=\bar{I}_{\ell}^{+}\right)\right)\right] \leqq \text {const. } \ell^{-d-\gamma} .
$$

From the assumption $h \in \tilde{\mathscr{G}}_{r}$ one has

$$
\left.\frac{\partial \hat{h}}{\partial Y_{\alpha}}\right|_{Y=z}=0, \quad \alpha \geqq 0,
$$

where $z_{\alpha}=E^{\mu_{z}}\left[I_{\alpha}\right]$. From the Taylor expansion we have

$$
\hat{h}(Y)=A(Y-z)+\text { higher order terms },
$$

where $A$ denote the quadratic terms. Assume that $\bar{I}_{\ell}^{+}$has a Gaussian distribution with mean $z$. Then

$$
E^{\mu_{z}}\left[\hat{h}\left(\bar{I}_{\ell}^{+}\right) ; \hat{h}\left(\bar{I}_{\ell}^{+}\right)\right] \sim E^{\mu_{z}}\left[Q\left(\bar{I}_{\ell}^{+}-z\right) ; Q\left(\bar{I}_{\ell}^{+}-z\right)\right] \sim \ell^{-2 d},
$$

and we have proved (6.23). The proof of (6.25) is elementary and involves only i.i.d. random variables. Since similar estimates were proved in [8] (Lemma 3.1) and in more details in [25] (Lemma 11.3), we omit the details.

\section{References}

1. Morrey, C.B.: On the derivation of the equations of hydrodynamics from Statistical Mechanics. Commun. Pure Appl. Math. 8, 279-290 (1955)

2. Olla, S., Varadan, S.R.S., Yau, H.T.: Hydrodynamical limit for a Hamiltonian system with weak noise. Commun. Math. Phys. 155, 523-560 (1993)

3. Nishida, T.: Fluid dynamical limit of the nonlinear Boltzmann equation to the level of the compressible Euler equation. Commun. Math. Phys. 61, 119-148 (1978)

4. Caflisch, R.E.: The fluid dynamic limit of the nonlinear Boltzmann equation. Commun. on Pure and Applied Math. 33, 651-666 (1980) 
5. Ukai, S., Asano, K.: The Euler limit and initial layer of the nonlinear Boltzmann equation. Hokkaido Math. J. 12, 303-324 (1983)

6. De Masi, A., Esposito, R., Lebowitz, J.L.: Incompressible Navier-Stokes and Euler limits of the Boltzmann equation. Commun. Pure Appl. Math. 42, 1189-1214 (1989)

7. Bardos, C., Golse, F., Levermore, D.: Fluid dynamical limits of kinetic equations I. Formal derivations. J. Stat. Phys. 63, 323-344 (1991)

8. Esposito, R., Marra, R., Yau, H.T.: Diffusive limit of asymmetric simple exclusion. Rev. Math. Phys. 6, 1233-1267 (1994)

9. Esposito, R., Marra, R.: On the derivation of the incompressible Navier-Stokes equation for hamiltonian particle systems. J. Stat. Phys. 74, 981-1004 (1993)

10. Yau, H.T.: Relative entropy and hydrodynamics of Ginsburg-Landau models. Lett. Math. Phys. 22, 63-80 (1991)

11. Frisch, U., d'Humieres, D., Hasslacher, B., Lallemand, P., Pomeau, Y., Rivet, J.P.: Lattice Gas Hydrodynamics in Two and Three Dimensions. Complex Systems 1, 649-707 (1987)

12. Fritz, J.: Stationary States and Hydrodynamics of FHP Cellular Automata. J. Stat. Phys. 77, 53-76 (1994)

13. De Masi, A., Esposito, R., Lebowitz, J.L., Presutti, E.: Hydrodynamics of Stochastic Cellular Automata. Commun. Math.Phys. 125, 127-145 (1989)

14. De Masi, A., Esposito, R., Presutti, E.: Kinetic limits of the HPP cellular automaton. J. Stat. Phys. 66, 403-464 (1992)

15. Landim, C., Yau, H.T.: Fluctuation-dissipation equation of asymmetric simple exclusion processes. Preprint 1996

16. Varadhan, S.R.S.: Nonlinear diffusion limit for a system with nearest neighbor interactions II. In: Proc. Taniguchi Symp., Kyoto (1990)

17. Spohn, H.: Large Scale Dynamics of Interacting Particles. New York: Springer-Verlag, 1991

18. Quastel, J.: Diffusion of color in the simple exclusion process. Commun. Pure Appl. Math. 40, 623-679 (1992)

19. Lu, S.L., Yau, H.T.: Spectral gap and Logarithmic Sobolev Inequality for Kawasaki and Glauber dynamics. Commun. Math. Phys. 156, 399-433 (1993)

20. Landim, C., Sethuraman, S., Varadhan, S.R.S.: Spectral gap for zero range dynamics. To appear in Annals of Prob., 1995

21. Xu, L.: Diffusion limit for the lattice gas with short range interactions. Thesis, New York University (1993)

22. Yau, H.T.: Logarithmic Sobolev Inequality for generalized simple exclusion processes. Preprint (1995)

23. Pomeau, Y., Resibois, P.: Time Dependent Correlation Functions and Mode-mode Coupling Theories. Phys. Rep. 19, 63-139 (1975)

24. Guo, M., Papanicolau, G.C., Varadhan, S.R.S.: Non-linear diffusion limit for a system with nearest neighbor interactions. Commun. Math. Phys. 118, 31-59 (1988)

25. Landim, C., Olla, S., Yau, H.T.: First order correction for the hydrodynamic limit of asymmetric simple exclusion processes in dimension $d \geqq 3$. Comm. Pure Appl. Math., to appear

26. Landim, C., Olla, S., Yau, H.T.: Some properties of the diffusion coefficient for asymmetric simple exclusion processes. Annals of Probability, to appear

27. Esposito, R., Marra, R., Yau, H.T.: Diffusive limit of the asymmetric simple exclusion: The Navier-Stokes correction. In: Nato ASI Series B: Physics 324, On three levels: MicroMeso and Macro-Approaches in Physics. M. Fannes-C. Maes-A. Verbeure, eds., 1994, pp. $43-53$ 
